\title{
Current Titles
}

National Center for Electron Microscopy

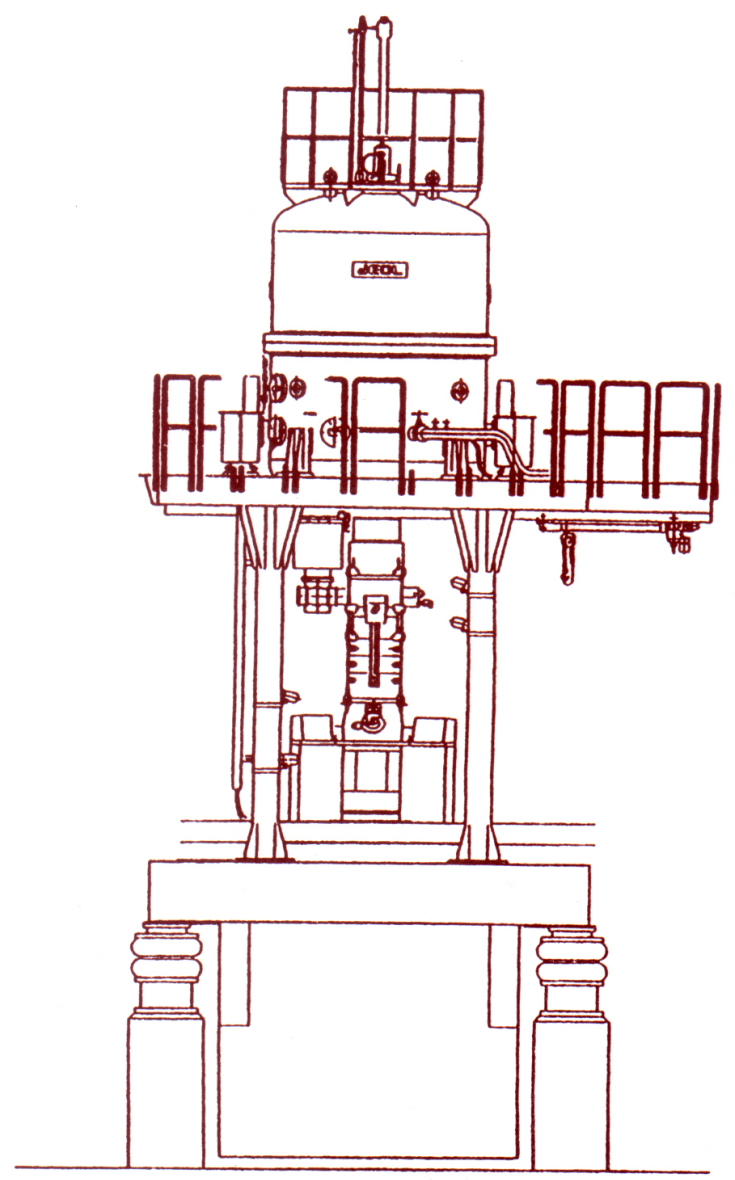




\section{DISCLAIMER}

This document was prepared as an account of work sponsored by the United States Government. While this document is believed to contain correct information, neither the United States Government nor any agency thereof, nor The Regents of the University of California, nor any of their employees, makes any warranty, express or implied, or assumes any legal responsibility for the accuracy, completeness, or usefulness of any information, apparatus, product, or process disclosed, or represents that its use would not infringe privately owned rights. Reference herein to any specific commercial product, process, or service by its trade name, trademark, manufacturer, or otherwise, does not necessarily constitute or imply its endorsement, recommendation, or favoring by the United States Government or any agency thereof, or The Regents of the University of California. The views and opinions of authors expressed herein do not necessarily state or reflect those of the United States Government or any agency thereof or The Regents of the University of California.

Ernest Orlando Lawrence Berkeley National Laboratory is an equal opportunity employer. 
Please send a reprint of the paper(s):

\begin{tabular}{|l|l|l|}
\hline Number & First Author & Title (first two words) \\
\hline & & \\
\hline & & \\
\hline & & \\
\hline & & \\
\hline & & \\
\hline & & \\
\hline & & \\
\hline
\end{tabular}

Name

Date

Affiliation

Address

Please send a reprint of the paper(s):

\begin{tabular}{|l|l|l|}
\hline Number & First Author & Title (first two words) \\
\hline & & \\
\hline & & \\
\hline & & \\
\hline & & \\
\hline & & \\
\hline & & \\
\hline & & \\
\hline
\end{tabular}

Name

Date

Affiliation

Address 
National Center for Electron Microscopy Berkeley Laboratory

1 Cyclotron Rd., Building 72

Berkeley CA 94720

National Center for Electron Microscopy

Berkeley Laboratory

1 Cyclotron Rd., Building 72

Berkeley CA 94720 


\title{
Current Titles
}

\author{
National Center for Electron Microscopy \\ Ernest Orlando Lawrence \\ Berkeley National Laboratory \\ University of California \\ Berkeley, California 94720
}

\section{April 2006}

PUB-719

For current information about the NCEM and a searchable version of publications visit our website: http://ncem.lbl.gov

The NCEM is supported by the Director, Office of Science U.S. Department of Energy under Contract No. DE-AC03-76SF00098. 
This booklet is published for those interested in current research being conducted at the National Center for Electron Microscopy. The NCEM is a DOE-designated national user facility and is available at no charge to qualified researchers. Access is controlled by an external steering committee. Interested researchers may contact Jane Cavlina, Administrator, at 510/486-6036.

Copies of available papers in print may be ordered by their publication number using the enclosed order cards. 


\title{
Extinction-Free Electron Diffraction Refinement of Bonding in $\mathrm{SrTiO}_{3}$
}

\author{
J. Friis, B. Jiang, J. Spence, K. Marthinsen, R. Holmstad
}

Acta Crystallogr. A59, 402, 2004

Accurate low-order Fourier coefficients of the crystal potential of $\mathrm{SrTiO}_{3}$ are measured by quantitative convergent-beam electron diffraction. The accuracy in the corresponding derived Xray structure factors is about $0.1 \%$ for the strong low-order reflections $\left(\sin \theta<0.3 \AA^{-1}\right)$. This accuracy is better than for conventional $\mathrm{X}$-ray diffraction and equivalent to the accuracy of the X-ray Pendellösung method. Combination of these structure factors with high-order X-ray diffraction measurements allows accurate bonding information to be obtained from a multipole model fitted to the experimental data. It is shown that Ti-O has a covalent component and that the Sr-O bond is mainly ionic. The role of Ti $3 d$ electrons in Ti-O bonding is also discussed.

\section{Si Nanowire Bridges in Microtrenches: Integration of Growth Into Device Fabrication}

R.R. He, D. Gao, R. Fan, A.I. Hochbaum, C. Carraro, R. Maboudian, P.D. Yang

Adv. Mater. 17, 2098, 2005

Silicon nanowire bridges are grown in prefabricated microtrenches on (110) silicon-on-insulator wafers. Silicon trenches are used as substrates during growth and probing electrodes after growth. This way, nanowire growth and device fabrication can be achieved simultaneously, providing a simple and rational way to realize nanowire-based integrated circuits. 


\title{
Catalysts from Self-Assembled Organometallic Block Copolymers
}

\author{
D. A. Durkee, H. B. Eitouni, E. D. Gomez, M. W. Ellsworth, A. T. Bell, N. P. Balsara
}

Adv. Mater. 2003, 2005

Heterogeneous catalysts were prepared by crosslinking the polyisoprene block of a microphase separated poly(vinyl ferrocenium triflate-block-isoprene) copolymer. The poly(vinyl ferrocenium triflate) moieties are responsible for catalytic activity while the polyisoprene block provides the support structure. The efficacy of the catalyst was analyzed by studying the Michael addition of ethyl-2-oxycyclopentane carboxylate and methyl vinyl ketone. The materials were studied via Transmission Electron Microscopy and the reaction rates were monitored by NMR spectroscopy. The activities obtained with our self-assembled heterogeneous catalyst were within experimental error of the rate of monomeric ferrocenium triflate as well as other homogeneous catalyst analogues. Our method for synthesizing catalysts is unique because the structures of both the support and active sites are controlled by molecular self-assembly.

\section{Single-Crystalline Diluted Magnetic Semiconductor GaN: Mn Nanowires}

H. J. Choi, H. K. Seong, J. Chang, K. I. Lee KI, Y. J. Park, J. J. Kim, S. K. Lee, R. R. He, K. T, P. D. Yang

Adv. Mater. 17, 1351, 2005

Current information technology relies on two independent processes: charge-based information processing (microprocessors) and spin-based data storage (magnetic hard drives). The prospect of simultaneously manipulating both charge and spin in a single semiconductor medium is provided by the exciting area of spintronics. Among many others, diluted magnetic semiconductors (DMSs) represent the most promising candidates for such applications. Herein we report on the magneto- and optoelectronic properties of single-crystalline diluted magnetic semiconductor nanowires Ga1-xMnxN ( $\mathrm{x}=0.01-0.09)$. These nanowires, which have diameters of similar to 10-100 $\mathrm{nm}$ and lengths of up to tens of micrometers, exhibit ferromagnetism with Curie temperatures $(\mathrm{T}(\mathrm{C}) \mathrm{s})$ above $300 \mathrm{~K}$ and magnetoresistances (MRs) up to $250 \mathrm{~K}$. Spindependent electron transport from single-nanowire transistors indicates the homogeneous nature of the ferromagnetic nanowires. 


\title{
Localized Functionalization of Single Nanopores
}

\author{
J. Nilsson, J. R. I. Lee, T. V. Ratto and S. E. Letant
}

Adv. Mater. 18, 427, 2006

Single nanopores can be fabricated using a combination of Focused Ion Beam drilling and local ion beam assisted oxide deposition. Letant and co-workers show how a silicon oxide ring can be grown around a single nanopore and how it can be functionalized with single stranded DNA using thiol-terminated linkers. Current voltage measurements performed at various stages of the fabrication process demonstrate the presence of DNA on the pore wall as well as the open nature of the pore. Future applications include specific detection of whole bio-organisms ranging in size from bacteria to viruses.

\section{A New Approach for Electron Tomography: Annular Dark-Field Transmission Electron Microscopy}

\author{
S. Bals, G. Van Tendeloo, C. Kisielowski
}

Adv. Mater. 18, 892, 2006

Transmission electron microscopy (TEM) images yield valuable information about the structure and chemistry of (in)organic materials. However, they "only" provide a 2D projection of a 3D object. Therefore, electron tomography was developed to reconstruct objects in three dimensions from a tilt series of TEM images. This technique is well accepted in the life sciences as a method used to study viruses or cells. The resolution in the reconstructions, however, is limited to a few nanometers, mainly because of the low resistance of biological materials to electron-beam damage. Electron-tomography techniques have recently been adopted by researchers in materials science. Here, atomic resolution could be achieved in principle. In practice, however, the resolution is still of the order of one to two nanometers. In this work, an alternative approach to electron tomography is proposed: electron tomography is combined with annular dark-field transmission electron microscopy (ADF-TEM). 


\title{
Nanotechnology in Catalysis
}

\section{T. Guo}

American Chemical Society Annual Meeting 3, (Chapter: Dual Catalytic Role of Co Nanoparticles in Bulk Synthesis of Si-Based Nanowires), 2005

We report here the results of the study of the catalytic roles of Co nanoparticles in the production of bulk-quantity Si-based nanowires (SiNW). Various growth conditions were explored, and the samples were characterized with Raman spectroscopy, electron microscopy, x-ray absorption spectroscopy, and photoluminescence. As a result of analyzing the growth conditions and growth mechanisms, we propose that Co nanoparticles, possibly in the form of Co silicides, play a dual catalytic role in the synthesis by helping to 1) make gaseous Si feedstock such as silane and 2) to catalyze the growth of SiNW.

\section{Ultrastructure, Aggregation-State, and Crystal Growth of Biogenic Nanocrystalline Sphalerite and Wurtzite}

\author{
J. W. Moreau, R. I. Web, J. F. Banfield
}

American Mineralogist 89, 950, 2004

High-resolution transmission electron microscope (HRTEM) data reveal that the earliest biologically induced metal sulfide precipitates are crystalline $\mathrm{ZnS}$ nanoparticles $1-5 \mathrm{~nm}$ in diameter. Nanocrystals of wurtzite are also present, consistent with a predicted size dependence for $\mathrm{ZnS}$ phase stability. The results highlight the importance of basic mineralogical information for accurate prediction and monitoring of long-term contaminant metal mobility and bioavailability in natural and constructed bioremediation systems. 


\title{
Transition-Metal Doped Zinc Oxide Nanowires
}

\author{
B. D. Yuhas, D. O. Zitoun, P. J. Pauzauskie, R. R. He, P. D. Yang
}

Angew. Chem. Int. Ed. 45, 420, 2006

The introduction of impurity atoms into semiconducting materials is the primary method for controlling the properties of the semiconductor, such as band gap or electrical conductivity. In particular, II-VI and III-V semiconductors that have been doped with transition metals are envisioned to be potential building blocks for spintronic devices. One such material is transitionmetal doped zinc oxide $\left(\mathrm{Zn}_{1-\mathrm{x}} \mathrm{M}_{\mathrm{x}} \mathrm{O}\right)$, which has been theoretically predicted to be ferromagnetic at room temperature. Although this system has been under experimental study for some time, the vast majority of research conducted on this material has been done on bulk crystals or thin films. There are very few reports on the fabrication of one-dimensional nanostructures of $\mathrm{Zn}_{1-\mathrm{x}} \mathrm{M}_{\mathrm{x}} \mathrm{O}$, and high-temperature, vapor-phase methods are employed in the syntheses. Although this approach has proven quite effective for the production of a multitude of nanoscale semiconductors, gas-phase syntheses are considerably limited in regards to homogeneous doping and alloying because of high-growth temperatures.

\section{V-shape Inversion Domains in InN Grown on c-Plane Sapphire}

\author{
J. Jasinski, Z. Liliental-Weber, H. Lu, W.J. Schaff
}

Appl. Phys. Lett. 85, 233, 2004

Inversion domains with a $\mathrm{V}$ shape were found to nucleate inside a $\mathrm{Mg}$-doped $\mathrm{InN}$ heteroepitaxial layer. They resemble Al-polarity domains, observed recently, in N-polarity AlN films. However, the angle between the sidewalls of the $\mathrm{V}$-shaped domain and the $\mathrm{c}$ axis differs in these two cases. In InN, this angle is almost two times bigger than that reported for AlN. The origin of V-shaped inversion domains in InN film is not yet clear. 


\title{
Control of Carbon Nanotube Morphology by Change of Applied Bias Field during Growth
}

\author{
L. H. Chen, J. F. AuBuchon, A. Gapin, C. Daraio, P. Bandaru, S. Jin, D. W. Kim, I. K. Yoo
}

Appl. Phys. Lett. 85, 5373, 2004

Carbon nanotube morphology has been engineered via simple control of applied voltage during dc plasma chemical vapor deposition growth. Below a critical applied voltage, a nanotube configuration of vertically aligned tubes with a constant diameter is obtained. Above the critical voltage, a nanocone-type configuration is obtained. The strongly field-dependent transition in morphology is attributed primarily to the plasma etching and decrease in the size of nanotubenucleating catalyst particles. A two-step control of applied voltage allows a creation of dualstructured nanotube morphology consisting of a broad base nanocone (similar to $200 \mathrm{~nm}$ dia.) with a small diameter nanotube (similar to $7 \mathrm{~nm}$ ) vertically emanating from the apex of the nanocone, which may be useful for atomic force microscopy.

\section{Effects of the Ratio of Hardness to Young's Modulus on the Friction and Wear Behavior of Bilayer Coatings}

\author{
W. Y. Ni, Y. T. Cheng, D. S. Grummon
}

Appl. Phys. Lett. 85, 4028, 2004

We present a study of the effects of the ratio of hardness to Young's modulus on the friction and wear behavior of layered composite coatings. Layered coating structures with the same surface coating but different interlayers were prepared by physical vapor deposition. We found that the ratio of hardness to Young's modulus plays an important role in determining the friction coefficient and wear resistance of layered composite coatings. A low friction coefficient and high wear resistance can be achieved in structures with high ratio of hardness to Young's modulus and moderately high hardness. 


\section{Phase-Change Recording Medium that Enables Ultrahigh-Density Electron- Beam Data Storage}

G. Gibson, A. Chaiken, K. Nauka, C. Yang, R. Davidson, A. Holden, R. Bicknell, B. Yeh, J. Chen, H. Liao, S. Subramanian, D. Schut, J. Jasinski, Z. Liliental-Weber

Appl. Phys. Lett. 86, 051902, 2005

An ultrahigh-density electron-beam-based data storage medium is described that consists of a diode formed by growing an $\mathrm{InSe} / \mathrm{GaSe}$ phase-change bilayer film epitaxially on silicon. Bits are recorded as amorphous regions in the InSe layer and are detected via the current induced in the diode by a scanned electron beam. This signal current is modulated by differences in the electrical properties of the amorphous and crystalline states. The success of this recording scheme results from the remarkable ability of layered III-VI materials, such as InSe, to maintain useful electrical properties at their surfaces after repeated cycles of amorphization and recrystallization.

\section{Electrical Properties of InAIP Native Oxides for Metal-Oxide-Semiconductor Device Applications}

Y. Cao, J. Zhang, X. Li, T.H. Kosel, P. Fay, D. Hall, X.B. Zhang, R. Dupuis, J. Jasinski, Z. Liliental-Weber

Appl. Phys. Lett. 86, 062105, 2005

Data are presented on the insulating properties and capacitance-voltage characteristics of metal-oxide-semiconductor (MOS) device-thickness (below $\sim 100 \mathrm{~nm}$ ) native oxides formed by wet thermal oxidation of thin InAlP epilayers lattice matched to GaAs. Low leakage current densities of $\mathrm{J}=1.4 / 10^{-9} \mathrm{~A} / \mathrm{cm}^{2}$ and $\mathrm{J}=8.7 / 10^{-11} \mathrm{~A} / \mathrm{cm}^{2}$ are observed at an applied field of $1 \mathrm{MV} / \mathrm{cm}$ for MOS capacitors fabricated with 17 and $48 \mathrm{~nm}$ oxides, respectively. Transmission electron microscopy images show that the In-rich interfacial particles, which exist in $110 \mathrm{~nm}$ oxides are absent in $17 \mathrm{~nm}$ oxide films. Quasistatic capacitance-voltage measurements of MOS capacitors fabricated on both n-type and p-type GaAs show that the InAlP oxide-GaAs interface is sufficiently free of traps to support inversion, indicating an unpinned Fermi level. These data suggest that InAlP native oxides may be a viable insulator for GaAs MOS device applications. 


\title{
Mechanical Elasticity of Single and Double Clamped Silicon Nanobeams
}

\author{
A. San Paulo, R. He, D. Gao, C. Carraro, J. Bokor, R. T. Howe, R. Maboudian, P. Yang,
}

Appl. Phys. Lett. 87, 053111, 2005

Atomic force microscopy has been used to characterize the mechanical elasticity of Si nanowires synthesized by the vapor-liquid-solid method. The nanowires are horizontally grown between the two facing $\mathrm{Si}(111)$ sidewalls of microtrenches prefabricated on a $\mathrm{Si}(110)$ substrate, resulting in suspended single and double clamped nanowire-in-trench structures. The deflection of the nanowires is induced and measured by the controlled application of normal forces with the microscope tip. The observed reversibility of the nanowire deflections and the agreement between the measured deflection profiles and the theoretical behavior of single and double clamped elastic beams demonstrate the overall beamlike mechanical behavior and the mechanical rigidity of the clamping ends of the nanowire-in-trench structures. These results demonstrate the potential of the nanowire-in-trench fabrication approach for the integration of VLS grown nanostructures into functional nanomechanical devices.

\section{Z-contrast and EELS Study of Passive Layer Formation at Ferroelectric $\mathrm{PbTiO}_{3} / \mathrm{Pt}$ Interfaces}

\author{
L. F. Fu, S. J. Welz, P. C. McIntyre, N.D. Browning
}

Appl. Phys. Lett. 87, 262904, 2005

Scanning transmission electron microscopy and electron energy loss spectroscopy have been applied to investigate the possible structural origins of ferroelectric polarization degradation at $\mathrm{PbTiO}_{3} / \mathrm{Pt}$ interfaces. The microscopic analysis revealed that an amorphous Ti-rich interfacial layer as well as nanometer size precipitates was formed at $\mathrm{PbTiO}_{3} / \mathrm{Pt}$ interfaces. The interfacial layer appears to form through decomposition of the $\mathrm{PbTiO}_{3}$ film due to a ferroelectric-electrode reaction during Pt deposition. The formation of the interfacial layer and precipitates could contribute to the polarization degradation typically observed for Pt-electroded $\mathrm{PbTiO}_{3}$-based ferroelectric capacitors. 


\title{
Modeling of Heterogeneous Precipitation of Iron in Silicon
}

\author{
A. Harahiltunen, H. Vainola, O. Anttila, M. Yli-Koski
}

Appl. Phys. Lett. 87, 151908, 2005

A predictive model is presented for the growth and dissolution of iron precipitates at oxygenrelated defects in silicon during thermal processing. The heterogeneous nucleation of iron is taken into account by special growth and dissolution rates, which are inserted into a set of modified chemical rate equations. This approach allows us to calculate the size distribution of iron precipitates and the residual iron concentration. By comparing the simulated results with experimental ones, it is proved that this model can be used to estimate the internal gettering efficiency of iron under a variety of processing conditions.

\section{Quantitative Copper Measurement in Oxidized p-type Silicon Wafers Using Microwave Photoconductivity Decay}

\author{
H. Vainola, E. Saarnilehto, M. Yli-Koski, A. Haarahiltunen, J. Sinkkonen, G. Berenyi, T. Pavelka
}

Appl. Phys. Lett. 87, 032109, 2005

We propose a method to measure trace copper contamination in $p$-type silicon using the microwave photoconductivity decay $(\mu$-PCD) technique. The method is based on the precipitation of interstitial copper, activated by high-intensity light, which results in enhanced minority carrier recombination activity. We show that there is a quantitative correlation between the enhanced recombination rate and the $\mathrm{Cu}$ concentration by comparing $\mu$-PCD measurements with transient ion drift and total reflection $\mathrm{x}$-ray fluorescence measurements. The results indicate that the method is capable of measuring $\mathrm{Cu}$ concentrations down to $10^{10} \mathrm{~cm}^{-3}$. There are no limitations to wafer storage time if corona charge is used on the oxidized wafer surfaces as the charge prevents copper outdiffusion. We briefly discuss the role of oxide precipitates both in the copper precipitation and in the charge carrier recombination processes. 


\section{Interfacial Reactions During GaN and AIN Epitaxy on 4H- and 6H-SiC(0001)}

M. Losurdo, P. Capezzuto, G. Bruno, A. Brown, K. Tong-Ho, Y. Changhyun, D.N. Zakharov, Z. Liliental-Weber

Appl. Phys. Lett. 86, 21920, 2005

Thin layers of $\mathrm{A} 1 \mathrm{~N}$ and $\mathrm{GaN}$ have been grown by plasma-assisted molecular beam epitaxy on $\mathrm{Si}$-face $4 \mathrm{H}$ - and $6 \mathrm{H}-\mathrm{SiC}(0001) /$ sub $\mathrm{Si} /$ substrates. The impact of the $\mathrm{SiC}$ surface preparation and oxide removal via a Ga deposition and desorption process on the chemistry and structure of the $\mathrm{GaN} / \mathrm{SiC}$ and $\mathrm{A} 1 \mathrm{~N} / \mathrm{SiC}$ interfaces, and on the $\mathrm{GaN} / \mathrm{SiC}$ subsurface reactivity is characterized. We also investigate the impact of this process on growth mode evolution.

\section{Size and Shape Evolution of Self-assembled Embedded Single Crystal $\alpha-F e$ Nanowires}

L. Mohaddes-Ardabili, H. Zheng, Q. Zhan, S. Y. Yang, R. Ramesh, L. Salamanca-Riba, M. Wuttig, S. B. Ogale

Appl. Phys. Lett. 87, 203110, 2005

The size and shape evolution of self-assembled ferromagnetic _-Fe nanowires is discussed. $-\mathrm{Fe}$ nanowires are formed by pulsed laser deposition of $\mathrm{La}_{0.5} \mathrm{Sr}_{0.5} \mathrm{FeO}_{3-\mathrm{x}}$ on single-crystal $\mathrm{SrTiO}_{3}$ (001) substrate in reducing atmosphere. The average diameter of the nanowires increases from $\mathrm{d}$ $\sim 4$ to $50 \mathrm{~nm}$ as the growth temperature increases from $\mathrm{T}=560$ to $840{ }^{\circ} \mathrm{C}$. Their in-plane shape evolves from circular to octahedral and square shape with [110] facets dominating as the growth temperature increases. A fitting to a theoretical calculation shows that the circular shape is stable when the diameter of the nanowires is smaller than $8 \mathrm{~nm}$. 


\title{
Direct Measurements of Water Adsorption Enthalpy on Hafnia and Zirconia
}

\author{
S.V. Ushakov, A. Navrotsky
}

Appl. Phys. Lett. 87, 164103, 2005

A commercial surface area analyzer and Calvet-type microcalorimeter were combined for measurements of heats of gas-solid interactions, providing enhanced resolution, flexibility and throughput compared to conventional methods. Integral adsorption enthalpies for half monolayer coverage on $\mathrm{HfO}_{2}$ and $\mathrm{ZrO}_{2}$ surfaces were found to be in the range -130-190 and -110-170 kJ per mole of gaseous $\mathrm{H}_{2} \mathrm{O}$ for differently prepared monoclinic phases and -70 and $-90 \mathrm{~kJ} / \mathrm{mole}$ for tetragonal phases from precipitation. The surface enthalpy for anhydrous tetragonal $\mathrm{ZrO}_{2}$ was derived as $1.23 \pm 0.04 \mathrm{~J} / \mathrm{m}^{2}$ from water adsorption and high-temperature solution calorimetry data.

\section{Pseudoelasticity of Martensitic Titanium-Nickel Shape-Memory Films Studied by In Situ Heating Nanoindentation and Transmission Electron Microscopy}

\author{
K. Komvopoulosa, X.-G. Ma
}

Appl. Phys. Lett. 87, 263108, 2005

Pseudoelasticity of shape-memory alloys has been commonly associated with reversible austenite-martensite phase transformation characterized by diffusionless, shear-induced atomic rearrangement. In situ heating nanoindentation and transmission electron microscopy results of this study demonstrate that martensitic TiNi films may also exhibit pseudoelasticity over a specific temperature range due to the reversible movement of the twin boundaries. The nanoscale deformation behavior of martensitic TiNi films was examined over certain ranges of normal load and temperature. The underlying mechanisms of the reversible twin boundary movement and the energy dissipated by pseudoelastic and elastic-plastic deformation in the TiNi films are interpreted in the context of in situ heating nanoindentation responses. The obtained experimental evidence is in agreement with a unified description of pseudoelasticity in which the origin of the restoring force responsible for the pseudoelastic behavior of shape-memory alloys is considered to be the formation of metastable pseudotwins. 


\title{
Quantifying Stoichiometry of Mixed-Cation-Anion III-V Semiconductor Interfaces at Atomic Resolution
}

\author{
K. Mahalingam, K. G. Eyink, G. J. Brown, D. L. Dorsey, C. F. Kisielowski, and A. Thust
}

Appl. Phys. Lett. 88, 091904, 2006

Employing the focal-series reconstruction technique in high-resolution transmission electron microscopy, we obtained true atomic resolution images of interfacial disorder within cation and anion sublattices across interfaces in an $\mathrm{InGaSb} / \mathrm{InAs}$ heterostructure. This enabled independent quantitative mapping of changes in the In-Ga and $\mathrm{As}-\mathrm{Sb}$ contents across interfacial regions similar to $0.6 \mathrm{~nm}$ in width. A comparison of the cation and anion sublattice images revealed that intermixing at the InGaSb-on-InAs interface is confined to the In-Ga sublattice. Also, atomic scale roughness within the As-Sb sublattice of the InAs-on-InGaSb interface was discerned. This approach is general, permitting atomic-scale compositional analysis of heterointerfaces with two species per sublattice.

\section{Atomic-Resolution Observations of Semicrystalline Intergranular Thin Films in Silicon Nitride}

\author{
A. Ziegler, J. C. Idrobo, M. K. Cinibulk, C. Kisielowski, N. D. Browning, R. O. Ritchie
}

Appl. Phys. Lett. 88, 4, 41919, 2006

Nanoscale intergranular films in doped silicon-nitride ceramics are known to markedly affect toughness and creep resistance. They are regarded as being fully amorphous, but are shown here to have a semicrystalline structure in a Ce-doped $\mathrm{Si}_{3} \mathrm{~N}_{4}$. Using two different but complementary high-resolution electron-microscopy methods, the intergranular atomic structure, imaged with sub-angstrom resolution, reveals that segregated cerium ions take very periodic positions, along the intergranular-phase/matrix grain interface and as a semicrystalline structure spanning the width of the intergranular phase. This result has broad implications for the understanding of the structure and role of the intergranular phase in enhancing the mechanical properties of ceramics. 


\title{
Consequences of Niobium Doping for the Ferromagnetism and Microstructure of Anatase Co: $\mathrm{TiO}_{2}$ Films
}

\author{
S. X. Zhang, S. B. Ogale, L. F. Fu, N. D. Browning, T. Venkatesan
}

Appl. Phys. Lett. 88, 012513, 2006

It is shown that dilute niobium doping has a significant effect on the ferromagnetism and microstructure of dilutely cobalt-doped anatase $\mathrm{TiO}_{2}$ films. Epitaxial films of anatase $\mathrm{TiO}_{2}$ with $3 \% \mathrm{Co}$, without and with $1 \%$ niobium doping were grown by pulsed-laser deposition at $875^{\circ} \mathrm{C}$ at different oxygen pressures. For growth at $10^{-5}$ Torr niobium doping suppresses the ferromagnetism, while it enhances the same in films grown at $10^{-4}$ Torr. High-resolution Zcontrast scanning transmission electron microscopy and electron energy loss spectroscopy show uniform surface segregation of cobalt-rich $\mathrm{Ti}_{1-\mathrm{x}-\mathrm{y}} \mathrm{Co}_{\mathrm{x}} \mathrm{Nb}_{\mathrm{y}} \mathrm{O}_{2-\delta}$ phase, but without cobalt metal clusters.

\section{Growth of Aligned Carbon Nanotubes on Carbon Microfibers by DC Plasma- Enhanced Chemical Vapor Deposition}

\author{
L. H. Chen, J. F. AuBuchon, I. C. Chen, C. Daraio, X. R. Ye, A. I. Gapin, S. Jin, C. M. Wang
}

Appl. Phys. Lett. 88, 033103, 2006

It is shown that unidirectionally aligned carbon nanotubes can be grown on electrically conductive network of carbon microfibers via control of buffer layer material and applied electric field during de plasma chemical vapor deposition growth. Ni catalyst deposition on carbon microfiber produces relatively poorly aligned nanotubes with significantly varying diameters and lengths obtained. The insertion of Ti $5 \mathrm{~nm}$ thick underlayer between Ni catalyst layer and $\mathrm{C}$ microfiber substrate significantly alters the morphology of nanotubes, resulting in much better aligned, finer diameter, and longer array of nanotubes. This beneficial effect is attributed to the reduced reaction between $\mathrm{Ni}$ and carbon paper, as well as prevention of plasma etching of carbon paper by inserting a Ti buffer layer. Such a unidirectionally aligned nanotube structure on an open-pore conductive substrate structure may conveniently be utilized as a high-surface-area base electrodes for fuel cells, batteries, and other electrochemical and catalytic reactions. 


\title{
Deforming Nanocrystalline Ni at Ultrahigh Strain Rates
}

Y. M. Wang, E. M. Bringa, J. M. McNaney, M. Victoria, A. Caro, A. M. Hodge, R. Smith, B. Torralva, B. A. Remington, C. A. Schuh, H. Jamarkani, M. A. Meyers.

Appl. Phys. Lett. 88, 061917, 2006

The deformation mechanism of nanocrystalline Ni (with grain sizes in the range of 30-100 nm) at ultrahigh strain rates $\left(>10^{7} \mathrm{~s}^{-1}\right)$ was investigated. A laser-driven compression process was applied to achieve high pressures $(20-70 \mathrm{GPa})$ on nanosecond timescales and thus induce highstrain-rate deformation in the nanocrystalline Ni. Postmortem transmission electron microscopy examinations revealed that the nanocrystalline structures survive the shock deformation, and that dislocation activity is a prevalent deformation mechanism for the grain sizes studied. No deformation twinning was observed even at stresses more than twice the threshold for twin formation in micron-sized polycrystals. These results agree qualitatively with molecular dynamics simulations and suggest that twinning is a difficult event in nanocrystalline $\mathrm{Ni}$ under shock-loading conditions.

\section{Recovery of Microindents in a Nickel-Titanium Shape-Memory Alloy: A "Self-Healing" Effect}

\author{
W. Y. Ni, Y. T. Cheng, D. S. Grummon
}

Appl. Phys. Lett. 80, 3310, 2006

The thermally induced recovery of microscopic deformation in a nickel-titanium shape-memory alloy was examined. Surface deformation was simulated by indenting the alloy in the martensite phase at room temperature using both spherical and pyramidal indenters. We show that deformation in spherical microindents can be almost completely reversed by moderate heating. Partial recovery was observed for pyramidal impressions formed by a Vickers indenter and the recovery ratio was independent of the indentation depth. The observations were rationalized using the concept of representative strain and maximum stress under the spherical and pyramidal indenters. 


\title{
Electrical Characterization of the Three-Way Gating Capability of Y- Junction Carbon Nanotube Transistors
}

\author{
J. Park, C. Daraio, S. Jin, P. R. Bandaru
}

Appl. Phys. Lett., 2006, In press

We report on the three-way gating capability of carbon nanotube based Y-junction morphologies. By measuring the output conductance and transconductance we conclude that the gating capability and the gain depend on the nanotube diameter and is electric field controlled. We propose a novel design for a Y-junction transistor, based on these principles, with the capability for tunable device characteristics.

\section{Theory of Stress Relaxation During Annealing of Ion Beam Synthesized Nanocrystals}

D.O. Yi, I.D. Sharp, Q. Xu, C.Y. Liao, J.W. Ager III, J. Beeman, Z. Liliental-Weber, K.M. Yu, D. Zakharov, E.E. Haller, D.C. Chrzan

APS, 17.13.1, 2004

Nanocrystals grown through ion beam synthesis have been found to be under appreciable compressive stresses. It is argued that these stresses arise from two fundamental sources: interface tension and aggregation stresses. Aggregation stresses are dynamic in origin. The aggregating atoms collect so rapidly that the growth rate of the nanocrystals exceeds the growth rate of the associated cavity in the host matrix. Subsequent annealing, then, can relax these aggregation stresses. We present a theory for this relaxation process that reflects the relevant elastic and surface energies. The relaxation is mediated by the diffusion of host matrix material away from the cavity containing the nanocrystal. The theory provides a good description of the relaxation of aggregation stresses in ion beam synthesized Ge nanocrystals grown in a silica matrix. This work is supported in part by the Department of Energy under contract No. DEAC03-76SF00098 and by the NSF Grant No. DMR-0109844. D.O. Yi Acknowledges the Luce Foundation for their support. 


\section{Direct Synthesis and Self-Assembly of Silicon Nanowires}

O. Englander, D. Christensen, J.B. Kim, L. Lin

ASME International Mechanical Engineering Congress and Exposition, 2004

The direct synthesis and self-assembly of silicon nanowires to yield a two-terminal, nano-tomicro integrated system has been demonstrated. The process takes advantage of localized heating to initiate and sustain a bottom up nanowire synthesis mechanism. As soon as the nanowire synthesis process is complete, the integrated system is ready for characterization of mechanical and electrical properties as well as functionalization for sensing applications. The process is CMOS compatible and eliminates the nano-to-micro contact formation process that is currently required of traditional processes.

\section{Nano-gap Piezoelectric Resonators for Mechanical RF Magnetic Field Modulation}

C. D. White, G. Piazza, P. J. Stephanou, A. P. Pisano

ASME International Mechanical Engineering Congress and Exposition, 4, 2005

Novel piezoelectric MEMS resonators with magnetic shielding have been developed to mechanically generate RF magnetic fields in the $\mathrm{MHz}$ frequency range from static magnetic fields. Thermally evaporated thin film nickel iron (NiFe) (10 to $20 \mathrm{~nm}$ ) was deposited with a saturation flux density of $0.5 \mathrm{~T}$, coercivity of $500 \mathrm{~A} / \mathrm{m}$ and a high relative permeability of $3.7 \mathrm{x}$ $10^{3}$. Aluminum nitride (AIN) resonators developed for high frequency operation, resonate in a contour mode to increase and decrease a gap surrounding the resonator. This gap, 200 to $350 \mathrm{~nm}$, is formed using a silicon sidewall deposition as a sacrificial spacer. Resonators with frequencies up to $60 \mathrm{MHz}$ have been fabricated and tested. 


\title{
Single Cell Electrophysiological Analysis via Sub-Micrometer Openings
}

\author{
S. Li, L. Lin
}

ASME International Mechanical Engineering Congress and Exposition, Proceedings of the MEMS Symposium, 2005

Single cell electrophysiological analyses were demonstrated via sub-micrometer openings using micro- and nano-machining technologies. In the prototype demonstration, a $6 \mathrm{G} f C$ seal resistance was achieved on a HeLa cell, a cervical cancer cell line with size between 10 20f_m, using the microfabricated electrophysiological system with opening size of $500 \mathrm{~nm}$ in diameter and the measured electrolyte pipette resistance is $200 \mathrm{k} f C \zeta$. In a second experiment on a vacuolar yeast cell of $3 \sim 5 f \_\mathrm{m}$ in size, using a device with $800 \mathrm{~nm}$ opening, a $500 \mathrm{M} f C ̧$ seal resistance was achieved and the measured electrolyte pipette resistance is $1 \mathrm{M} f C$.

\section{A Transmission Electron Microscopy Study of Mineralization in Age-Induced Transparent Dentin}

A. Porter, R. Nalla, A. E. Porter, R. Nalla, A. Minor, J. Jinschek, C. Kisielowski, V. Radmilovic, J. Kinney, A. Tomsia, R.O. Ritchie

Biomaterials, 26, 36, 7650, 2005

It is known that fractures are more likely to occur in altered teeth, particularly following restoration or endodontic repair; consequently, it is important to understand the structure of altered forms of dentin, the most abundant tissue in the human tooth, in order to better define the increased propensity for such fractures. Transparent (or sclerotic) dentin, wherein the dentinal tubules become occluded with mineral as a natural progressive consequence of aging, is one such altered form. In the present study, high-resolution transmission electron microscopy is used to investigate the effect of aging on the mineral phase of dentin. Such studies revealed that the intertubular mineral crystallites were smaller in transparent dentin, and that the intratubular mineral (larger crystals deposited within the tubules) was chemically similar to the surrounding intertubular mineral. Exit-wave reconstructed lattice-plane images suggested that the intratubular mineral had nanometer-size grains. These observations support a "dissolution and reprecipitation" mechanism for the formation of transparent dentin. 


\title{
Age-Related Transparent Root Dentin: Mineral Concentration, Crystallite Size, and Mechanical Properties
}

\author{
J. H. Kinney, R. K. Nalla, J. A. Pople, T. M. Breunig, R. O. Ritchie
}

Biomaterials, 26, 3363, 2005

Many fractures occur in teeth that have been altered, for example restored or endodontically repaired. It is therefore essential to evaluate the structure and mechanical properties of these altered dentins. One such altered form of dentin is transparent (sometimes called sclerotic) dentin, which forms gradually with aging. The present study focuses on differences in the structure and mechanical properties of normal versus transparent dentin. The mineral concentration, as measured by X-ray computed microtomography, was significantly higher in transparent dentin, the elevated concentration being consistent with the closure of the tubule lumens. Crystallite size, as measured by small angle X-ray scattering, was slightly smaller in transparent dentin, although the importance of this finding requires further study. The elastic properties were unchanged by transparency; however, transparent dentin, unlike normal dentin, exhibited almost no yielding before failure. In addition, the fracture toughness was lowered by roughly $20 \%$ while the fatigue lifetime was deleteriously affected at high stress levels.

\section{Effect of Aging on the Toughness of Human Cortical Bone: Evaluation by R- curves}

\author{
R. K. Nalla, J .J. Kruzic, J. H. Kinney, R.O. Ritchie
}

Bone, 35, 1240, 2004

The present study describes ex vivo fracture experiments to quantitatively assess the effect of aging on the fracture toughness properties of human cortical bone in the longitudinal direction. Because cortical bone exhibits rising crack-growth resistance with crack extension, unlike most previous studies, the toughness is evaluated in terms of resistance-curve (R-curve) behavior, measured for bone taken from wide range of age groups (34-99 years). Using this approach, both the ex vivo crack-initiation and crack-growth toughness are determined and are found to deteriorate with age; the initiation toughness decreases some $40 \%$ over 6 decades from 40 to 100 years, while the growth toughness is effectively eliminated over the same age range. The reduction in crack-growth toughness is considered to be associated primarily with a degradation in the degree of extrinsic toughening, involving crack bridging in the wake of the crack. 


\title{
Energetics of Mesoporous Silica: Investigation into Pore Size and Symmetry
}

\author{
O. Trofymluk, A.A. Levchenko, S.H. Tolbert, A. Navrotsky
}

Chem. Mater. 17, 3772, 2005

Two families of calcined highly ordered mesoporous silicas, designed as M41S (MCM-41 and MCM-48) and SBA-n (SBA-15 and SBA-16), are investigated in a wide range of pore sizes from 2.1 to $26.4 \mathrm{~nm}$ by high-temperature oxide melt solution calorimetry using lead borate solvent at $974 \mathrm{~K}$. These data are consistent with and extend our earlier studies of zeolite microporous and of mesoporous silicas. The formation enthalpies observed are 19.0-31.4 kJ/mol less exothermic than that of quartz and correlate linearly with pore size for a given structure type. Small- and wide-angle X-ray scattering, nitrogen adsorption (BET), thermogravimetric analysis, and ${ }^{29} \mathrm{Si}$ NMR are employed to give insight into structure and symmetry. The enthalpy differences among samples are discussed in terms of symmetry and structural (point and ring) defects in the materials.

\section{Energetics of Bulk and Nano-Akaganeite, Beta-FeOOH: Enthalpy of Formation, Surface Enthalpy and Enthalpy of Water Adsorption}

\author{
L. Mazeina, S. W. Deore, A. Navrotsky
}

Chem. Mater. 18, 1830, 2006

\begin{abstract}
Akaganeite, $\beta-\mathrm{FeOOH}$, is a commonly occurring ferric mineral in the environment and is a sorbent, ion exchanger, and catalyst. It is often fine-grained (nanophase) and frequently contains excess water. Its enthalpy of formation was studied by solution calorimetry in aqueous $\mathrm{HCl}$. The enthalpy of water adsorption was studied by a new calorimetric technique combining a Calvet microcalorimeter and an automated gas dosing system, used for surface adsorption measurements. Akaganeite samples with surface areas of $30-280 \mathrm{~m}^{2} / \mathrm{g}$ were used. Sample characterization was performed by X-ray powder diffraction, Fourier transform infrared spectroscopy, thermogravimetric analysis, Brunauer-Emmett-Teller method, scanning electron microscopy, and transmission electron microscopy. By adsorbing water, akaganeite decreases its effective surface enthalpy from $0.44 \mathrm{~J} / \mathrm{m}^{2}$ to $0.34 \mathrm{~J} / \mathrm{m}^{2}$. The enthalpy of formation of akaganeite can vary by $10-12 \mathrm{~kJ} / \mathrm{mol}$ as a function of the surface area. Thus, the standard enthalpy of formation and surface enthalpy of akaganeite are between those of goethite and lepidocrocite.
\end{abstract}




\title{
Wave-Function Engineering: from Quantum Wells to Near-Infrared Type-II Colloidal Quantum Dots Synthesized by Layer-by-Layer Colloidal Epitaxy
}

\author{
J. J. Li, J. M. Tsay, X. Michalet, S. Weiss
}

Chem. Phys. 318, 82, 2005

We review the concept and the evolution of bandgap and wave-function engineering, the seminal contributions of Dr. Chemla to the understanding of the rich phenomena displayed in epitaxially grown quantum confined systems, and demonstrate the application of these concepts to the colloidal synthesis of high quality type-II CdTe/CdSe quantum dots (QDs) using Successive Ion Layer Adsorption and Reaction (SILAR) chemistry. Transmission Electron Microscopy (TEM) reveals that $\mathrm{CdTe} / \mathrm{CdSe}$ can be synthesized layer by layer, yielding particles of narrow size distribution. Photoluminescence emission and excitation spectra reveal discrete type-II transitions, which correspond to energy lower than the type-I bandgap. The increase in the spatial separation between photoexcited electrons and holes as a function of successive addition of CdSe monolayers was monitored by photoluminescence lifetime measurements. Systematic increase in lifetimes demonstrates the high level of wavefunction engineering and control in these systems.

\section{Sodium Meta-Autunite Colloids: Synthesis, Characterization and Stability}

\author{
Z. Zheng, J.Wan, X. Song, T.K. Tokunaga
}

Colloids Surf. A 274, 48, 2006

Waste forms of uranium (U) such as those in the United States Department of Energy's Hanford Site, often contain high concentrations of sodium and phosphorus. Low solubility sodium uranyl phosphates such as sodium meta-autunite have the potential to form mobile colloids that can facilitate transport of this radionuclide. To understand the stability behavior of uranyl phosphate colloids, we synthesized sodium meta-autunite colloids and characterized their morphology, chemical composition, structure, dehydration, and surface charge. The stability of these synthetic, plate-shaped colloids was tested with respect to time and $\mathrm{pH}$. We observed the highest aggregation rate to be at $\mathrm{pH} \mathrm{3}$, indicating a higher stability of colloid dispersion under neutral and alkaline $\mathrm{pH}$ conditions. The synthetic colloids are all negatively charged, and no isoelectric points were found over a $\mathrm{pH}$ range of 3 to 9 . The high stability of sodium meta-autunite colloids under neutral-alkaline conditions suggests that formation of sodium meta-autunite colloids can enhance the transport of $\mathrm{U}$ in $\mathrm{U}$-contaminated sediments. 


\title{
Formation of $<111>$ Fiber Texture in $\beta$-SiC Films Deposited on $\mathrm{Si}(100)$ Substrates
}

\author{
V. Radmilovic, U. Dahmen, D. Gao, C.R. Stoldt, C. Carraro and R. Maboudian \\ Diamond and Related Materials, 2006, In press
}

The evolution of the morphology and texture of 3C-SiC films grown by chemical vapor deposition (CVD), using 1,3-disilabutane as precursor, on $\mathrm{Si}(100)$ substrates is investigated by transmission electron microscopy. Films were found to exhibit a columnar grain structure with a strong $<111>$ fiber texture and a high density of stacking faults and twins. The columnar grains do not originate at the substrate surface but on a buffer layer about 3 to $5 \mathrm{~nm}$ thick, consisting of interconnected 3D-islands that initiate as epitaxial nuclei. The change from $<100>$ epitaxial islands to $<111>$ columnar grains can be understood in terms of anisotropic growth rates and multiple twinning. The observed $<111>$ fiber texture, faulted substructure, faceted surface morphology and carbon enrichment of the growth surface are in agreement with the proposed growth model.

\section{Encyclopedia of Nanoscience and Nanotechnology, Vol. 2}

\author{
A.V. Sergueeva, N.A. Mara, A.K. Mukherjee
}

Deformation Behavior of Nanocrystalline Materials, 305-316, 2004

This chapter consists of an overview of the mechanical properties work conducted on nanocrystalline metals at both room and elevated temperatures. The correlation between microstructure and properties is emphasized in systems that range from pure Ti to intermetallics such as $\mathrm{Ni}_{3} \mathrm{Al}$. 


\title{
ZnO Nanowires Based UV Photodiodes
}

\author{
L. Luo, Y. Zhang, S.S. Mao, L. Lin
}

18th IEEE Micro Electro Mechanical Systems Conference, 427, 2005

An n-type zinc oxide $(\mathrm{ZnO})$ nanowires and p-type silicon heterojunction has been successfully constructed to demonstrate UV (ultra-violet) photodiodes. The prototype device consists of unintentionally doped n-type $\mathrm{ZnO}$ nanowire arrays in the range of 70 120 nanometers in diameter fabricated by the bottom-up vapor-liquid-gas growth process. The nanowires are grown on top of a (100) p-silicon substrate with a doping level of $\sim 10^{\wedge} 18 \mathrm{~cm}^{\wedge}-3$ to form the heterojunction. The isolation is achieved using spin-on glass that also works as the foundation of the top electrode. The current-voltage characteristics show the typical rectifying behavior, and the photodiode exhibits response of $0.07 \mathrm{~A} / \mathrm{W}$ for UV light $(365 \mathrm{~nm})$ under a $20 \mathrm{~V}$ of reverse bias.

\section{Electrochemical Synthesis of Functionalized Nickel Oxide Nanowires}

\author{
T. S. Mintz, Y. V. Bhargava, S. A. Thorne, R. Chopdekar, V. Radmilovic, Y. Suzuki, T.M. Devine
}

Electrochemical and Solid State Letters 8, D26, 2005

Bulk quantities of self-assembled, functionalized nickel oxide nanowires (lengths $20 \mu \mathrm{m}$; mean diameter $25 \mathrm{~nm}$ ) have been grown by a novel electrochemical method consisting of multistep oxidation of $\mathrm{Ni}-\mathrm{Cr}$ alloys in high-temperature, pressurized water. Nanowires form when growth results in a deficit of $\mathrm{OH}-$ in the electrolyte ahead of the growing NiO. The compositional gradient breaks up the planar $\mathrm{NiO} /$ water interface into a cellular interface, resulting in the growth of $\mathrm{NiO}$ nanowires. 


\title{
Detection of Iron Contamination in Internally Gettered P-Type Silicon Wafers by Lifetime Measurements
}

\author{
A. Haarahiltunen, H. Vainola, M. Yli-Koski, E. Saarnilehto, J. Sinkkonen
}

Electrochemical Society Proceedings, High Purity Silicon VIII, 05, 135, 2004

High Purity Silicon VIII, 05, 135-145, 2004We have studied the applicability of standard lifetime methods (SPV, _ PCD) to detect iron in internally gettered p-type silicon wafers. Conventional high - low - high anneals were performed to produce a series of wafers with varying denuded zone (DZ) width and oxygen precipitate density. The wafers were intentionally iron contaminated to a level of about $1-2 \mathrm{E} 13 \mathrm{~cm}^{-3}$. Different kinds of gettering treatments were applied to obtain a wide range of dissolved iron concentrations. The results indicate that the iron concentration down to $1 \mathrm{E} 11 \mathrm{~cm}^{3}$ can be quantitatively measured in internally gettered wafers by lifetime methods. In internally gettered wafers _-PCD has lower detection limit for iron than SPV but in both cases the detection limit depends on the internal gettering process. The reference measurements were done by DLTS.

\section{Enhancement of Internal Gettering Efficiency of Iron by Low Temperature Nucleation}

\author{
H.Vainola, A. Haarahiltunen, E. Saarnilehto, M. Yli-Koski, J. Sinkkonen
}

Electrochemical Society Proceedings, High Purity Silicon VIII, 05, 160, 2004

In order to better understand internal gettering of iron in silicon at low supersaturation, an experimental study of iron gettering with different annealing profiles was performed. Special attention was paid to the effect of iron nucleation at low temperatures. The results reveal that gettering can be efficient even after 30 minutes anneal at $700^{\circ} \mathrm{C}$ if the wafers go through a lowtemperature region before the actual gettering anneal. In the simplest case the low-temperature nucleation can be realized by a fast withdrawal of the wafers out of the furnace. The density of oxygen precipitates seemed to have only a minor impact on the gettering efficiency indicating that not every oxygen precipitate related gettering site is an active sink for iron. 


\title{
Carbon Cryogel as Support of Platinum Nano-sized Electrocatalyst for the Hydrogen Oxidation Reaction
}

\author{
B.M. Babic, Lj. M. Vracar, V.R. Radmilovic, and N.V. Krstajic
}

Electrochimica Acta, 51, 3820, 2006

The kinetics of hydrogen oxidation reaction was studied in perchloric acid solution on carbonsupported Pt nanoparticles using the rotating disk electrode technique. Carbon cryogel and commercial carbon black. Vulcan XC-72 was used as catalyst supports. Pt/C catalysts were prepared by a modified polyol synthesis method in an ethylene glycol (EG) solution. Considerable effect has been observed for the specific surface area of carbon support on the fundamental properties of $\mathrm{Pt} / \mathrm{C}$ catalyst, such as catalyst particle size distribution and dispersion as well as catalytic activity for the oxidation of hydrogen. X-ray diffraction (XRD) and transmission electron microscopy (TEM) images show that the particle size of the catalyst decreases with the increase of specific surface area of carbon support. Cyclic voltammetry (CV) was used for determination of the actual exposed surface area of catalyst particles. It was found that Pt catalyst prepared by using the novel carbon material displayed better hydrogen electrochemical oxidation activity than the catalyst prepared by using Vulcan XC-72.

\section{Development of the Pattern Generator for the Maskless Micro-beam Reduction Lithography Systems}

\author{
X. Jiang, K. N. Leung
}

49th International Conference on Electron, Ion and Photon Beam Technology and Nanofabrication, 2005

A proof-of-concept maskless ion beam lithography machine called Maskless Micro-beam Reduction Lithography (MMRL) has been developed in Lawrence Berkeley National Laboratory. MMRL can work in either the projection mode or the maskless mode. Sub-100 nm resolution has been demonstrated recently on the prototype MMRL system in a 10x reduction projection mode. A PMMA resist exposure result is shown in fig. 2. Pattern generators, which can individually switch ion beamlets on and off, are being fabricated in the microfabrication laboratory of UC-Berkeley. By employing the switchable pattern generator on the beam-forming electrode plane and combining the scanning of the high precision stages, MMRL can perform multiple-beam maskless lithography and offer a possible low-cost solution for the advanced ASIC fabrication 


\title{
Relation Between Structural and Optical Properties of $\operatorname{InN}$ and $\operatorname{In}_{\mathbf{x}} \mathbf{G a}_{1-\mathrm{x}} \mathrm{N}$ Thin Films
}

\author{
Z. Liliental-Weber, J. Jasinski and D.N. Zakharov
}

ICPS, Flagstaff, 2004

Transmission Electron Microscopy (TEM) and optical measurements obtained from $\mathrm{InN}$ and $\mathrm{In}_{1}$ ${ }_{x} \mathrm{Ga}_{\mathrm{x}} \mathrm{N}$ films $(0<\mathrm{x}<0.54)$ grown by Molecular Beam Epitaxy are presented. Energy gaps measured by absorption, PR, and PL for InN films grown on c-plane $\mathrm{Al}_{2} \mathrm{O}_{3}$ were in the range of $0.7 \mathrm{eV}$. No In or other inclusions were observed in these films, ruling out the possibility of a strong Mie scattering mechanism. In the $\mathrm{In}_{1-\mathrm{x}} \mathrm{Ga}_{\mathrm{x}} \mathrm{N}$ films the relationship between the structural properties and the optical properties, in particular the presence or absence of a Stokes shift between absorption and PL, is discussed. TEM studies show that high quality layers do not have a Stokes shift. Some films had compositional ordering; these films also showed a shift between absorption edge and luminescence peak.

\section{In Situ Characterization of Ge Nanocrystals at Elevated Temperatures}

I.D. Sharp, D.O. Yi, Q. Xu, C.Y. Liao, J.W. Beeman, Z. Liliental-Weber, K.M. Yu, D.N. Zakharov, J.W. Ager III, D.C. Chrzan, E.E. Haller

ICPS-27, Flagstaff, 2004

We present in situ electron diffraction data indicating that Ge nanocrystals embedded in a silica matrix can be solid at temperatures exceeding the bulk Ge melting point. Supercooling is observed when returning from temperatures above the melting point of the Ge nanocrystals. Since melting point hysteresis is observed, it is not clear if nanoclusters are solid or liquid during the initial growth process. Raman spectra of as-grown nanocrystals give a measure of compressive stress and in-situ Raman spectroscopy further confirms the presence of crystalline Ge above $800^{\circ} \mathrm{C}$. 


\title{
Fabrication of $\mathrm{GaN}_{\mathrm{x}} \mathrm{As}_{1-\mathrm{x}}$ Quantum Structures by Focused Ion Beam Patterning
}

K. Alberi, A. Minor, M.A. Scarpulla, S.J. Chung, D.E. Mars, K.M. Yu, W. Walukiewicz, O.D. Dubon

ICPS-27, 2004

A novel approach to the fabrication of $\mathrm{GaN}_{\mathrm{x}} \mathrm{As}_{1-\mathrm{x}}$ quantum dots and wires via ion beam patterning is presented. Photomodulated reflectance spectra confirm that $\mathrm{N}$ can be released from the As sublattice of an MBE-grown $\mathrm{GaN}_{\mathrm{x}} \mathrm{As}_{1-\mathrm{x}}$ film by amorphization through ion implantation followed by regrowth upon rapid thermal annealing (RTA). Amorphization may be achieved with a focused ion beam (FIB), which is used to implant Ga ions in patterned lines such that annealing produces $\mathrm{GaAs}$ regions within a $\mathrm{GaN}_{\mathrm{x}} \mathrm{As}_{1-\mathrm{x}}$ film. The profiles of these amorphized lines are dependent upon the dose implanted, and the film reaches a damage threshold during RTA due to excess Ga. By altering the FIB implantation pattern, quantum dots or wires may be fabricated.

\section{Directed Assembly of Ge Islands Grown on Au-Patterned Si(100)}

\author{
J. T. Robinson, J. A. Liddle, A. M. Minor, V. Radmilovic, O. D. Dubon
}

ICPS-27, 2004

We have achieved two-dimensional alignment of Ge islands grown by molecular beam epitaxy on $\mathrm{Au}$ patterned $\mathrm{Si}(100)$. Arrays of Au dots are patterned by electron-beam evaporation of $1 \mathrm{~nm}$ of $\mathrm{Au}$ through a stencil mask. For a square array of Au dots, a two-dimensional square lattice consisting of $\mathrm{Ge}$ islands less than $100 \mathrm{~nm}$ in height extending over hundreds of microns has been realized. Surprisingly, the Ge islands are square-based, $\{111\}$-side- and (100)-top-faceted truncated pyramids and grow at the centers of the squares formed by the Au-dot arrays. This novel growth process is a versatile method to manipulate island growth kinetics and thereby direct their assembly over large areas. 


\title{
GaN Grown in Polar and Non-Polar Directions
}

\author{
Z. Liliental-Weber, J. Jasinski, D.N. Zakharov
}

ICSSC and PCCG-7, Crystal Researching and Technology, 2004

In this paper; defects formed in GaN grown by different methods are reviewed. thin GaN films were grown on c-, m- and a-planes on a number of substrates and typical defects as characterized by transmission electron microscopy are described. For polar epilayers grown on c-plane sapphire the typical defects are dislocations (edge, screw and mixed). The lowest dislocation density was obtained for homoepitaxial growth using molecular beam epitaxy (MBE) or hydride vapor phase epitaxy (HVPE). In both cases, the cores are full. In the layers grown by HVPE the dislocations are decorated by pinholes stacked on top of each other. These pinholes are empty inside and their formation is attributed to impurities (oxygen) present in these layers. In these layers Ga-rich cores have been found. These were not observed in the layers grown by MBE on the top of the HVPE templates. Epilayers grown in non-polar directions ( $\mathrm{m}$ - or a-plane) have a high density of planar defects (stacking faults) terminated by partial dislocations. The majority of these faults are formed at the interface with the substrate and propagate to the sample surface.

\section{Molybdenum-Gate $\mathrm{HfO}_{2} \mathrm{CMOS}$ FinFET Technology}

\author{
D. Ha, H. Takeuchi, Y. K. Choi, T. J. King, W. P. Bai, D. L. Kwong, A. Agarwal, M. Ameen
}

International Electron Devices Meeting, 643, 2004

CMOS FinFETs with molybdenum gate and $\mathrm{HfO}_{2}$ gate dielectric are reported. By tuning the gate work function via nitrogen implantation and employing a narrow fin width, low values of threshold voltage $(0.28 /-0.17 \mathrm{~V})$ and sub-threshold swing $(67.5 / 62.5 \mathrm{mV} / \mathrm{dec})$ were achieved. The use of $\mathrm{HfO}_{2}$ rather than $\mathrm{SiO}_{2}$ as the gate dielectric reduces the gate leakage current density by several orders of magnitude, for EOT in the range 1.75-1.95 nm. The observed weak temperature dependence for both electron and hole mobilities $\left(\mathrm{i}_{\mathrm{eff}} \sim \mathrm{T}^{-0.95}\right)$ is ascribed to soft phonon scattering. 


\title{
Large Grain Poly-Si Thin Films by Metal Induced Crystallization of a-Si:H
}

\author{
M. A. Albarghouti, H. H. Abu-Safe, H. A. Naseem, W. D. Brown, M. M. Al-Jassim, K M. Jones
}

IEEE PVSC 31st Conference, 31st IEEE Conference, 1070, 2005

Large grain poly-Si thin films on glass substrates have been successfully fabricated. The film thickness was $400 \mathrm{~nm}$ and the grains sizes were in excess of several microns. The films were fabricated using aluminum-induced crystallization of a-Si:H in the presence of a silicon oxide layer at the $\mathrm{Al} / \mathrm{a}-\mathrm{Si}: \mathrm{H}$ interface. The a-Si:H film was deposited on glass substrates and the annealing temperatures and annealing times were kept below $450^{\circ} \mathrm{C}$ and 30 minutes, respectively. The resulting poly-Si was heavily Al-doped.

\section{Application of Exit-Plane Wave Functions in High-Resolution Transmission Electron Microscopy for Quantitative Analysis of III-V Semiconductor Interfaces}

\author{
K. Mahalingam, K. Eyink, G. J. Brown, D. L. Dorsey
}

Int. J. Nano Science 3, 723, 2004

The application of exit-plane wave function (EPWF) images for an atomic-scale compositional mapping of interfaces in the GaAs-AlAs system is investigated. Image simulations based on the multislice algorithm were performed to obtain EPWF images over a wide range of compositions and specimen thicknesses. By employing the method of factorial analysis of correspondence to the phase-component EPWF images, a simple procedure for quantifying the composition across an interface is proposed. A test of this procedure on a simulated EPWF-phase image of $\mathrm{A} 10.6 \mathrm{Ga} 0.4 \mathrm{As} / \mathrm{GaAs} / \mathrm{Al}_{0.6} \mathrm{Ga}_{0.4} \mathrm{As}$ structure, with atomically abrupt and graded interfaces, yields composition profiles which are in good agreement with the model-profiles. 


\title{
Optical, Electrical, and Diffusion Properties of Hafnium and Zirconium in Single Crystal Silicon
}

\author{
R. Sachdeva, A. A. Istratov, T. Radetic, X. Xu, P. N. K. Deenapanray, E.R. Weber \\ International Conference on Defects in Semiconductors, 2005
}

A study of optical, electrical, and diffusion properties of $\mathrm{Hf}$ and $\mathrm{Zr}$ in silicon is presented. Photoluminescence spectra were observed in Hf implanted silicon. Isotope substitution confirms that the observed signal is Hf-related. Several deep level defects were found for $\mathrm{Hf}$ in both the upper and lower half of silicon band gap, and their parameters were tabulated. Dominant defect in deep level spectra was determined to be a donor. Diffusion study of $\mathrm{Zr}$ implanted samples indicated that $\mathrm{Zr}$ tends to diffuse out to the surface. Outdiffusion and precipitation of $\mathrm{Zr}$ which was found to form platelets, as confirmed by transmission electron microscopy, does not allow the traditional diffusion models be used; however, an estimate of $\mathrm{Zr}$ diffusivity was performed, yielding a value of $2.8 \times 10^{-15} \mathrm{~cm}^{2} / \mathrm{s}$ at $1100^{\circ} \mathrm{C}$.

\section{Characterization of as-Deposited Crystalline NiTi Thin Films}

\author{
D. Bronfenbrenner, V. Radmilovic, S. A. McHugo, R. Gronsky
}

International Conference on Shape Memory and Superelastic Technologies, 2006

As-deposited crystalline NiTi thin films are analyzed by transmission electron microscopy, x-ray diffraction, and differential scanning calorimetry to determine their dominant phases, microstructure, and transformation behavior as a function of sputter deposition parameters. It is found that the NiTi films are in the twinned martensite phase at room temperature with an average grain size of $\sim 100 \mathrm{~nm}$ and a columnar microstructure. 


\title{
An Experimental Investigation on Cyclic Plastic Deformation and Substructures of Polycrystalline Copper
}

\author{
Y. Jiang, J. Zhang
}

Int. J. of Plasticity 21, 2191, 2005

Cyclic deformation under proportional and nonproportional loading of a textured copper was experimentally studied, and the results were compared with those of texture-free copper with the same grain size. The texture had a great influence on the equivalent cyclic stress-strain (CSS) curves under proportional loading but insignificant influence on the CSS curves under nonproportional loading. By comparing the slip patterns on the specimen surface and dislocation substructures under proportional and nonproportional loading, the mechanism of nonproportional hardening was discussed. Nonproportional hardening is the result of enhanced activated slip systems and more uniform activation of slip systems due to the rotation of maximum shear stress under nonproportional loading. A linear relationship existed between the saturation equivalent stress magnitude and the reciprocal of the diameter of the dislocation cells. Such a relationship was independent of the loading modes and texture. The saturation stress magnitude was related to the bowing stress of screw dislocations in the interior area of dislocation cells.

\section{ZnO-GaN Tunnel Junction for Transparent Ohmic Contacts to p-GaN}

E. Kaminska, a. Piotrowska, K. Golaszewska, R. Kruszka, A. Kuchuk, J. Szade, A. Winiarski, J. Jasinski, Z. Liliental-Weber

\section{J. Alloys Compd. 371, 129, 2004}

The fabrication procedure of transparent $\mathrm{n}+-\mathrm{ZnO}-\mathrm{p}-\mathrm{GaN}$ ohmic junctions has been described. The influence of consecutive technological steps on the electrical, structural and electronic properties of the junction has been studied. The results indicate that the predeposition of $\mathrm{Au}$ nucleation film plays a crucial role for the final contact properties. The ohmic behaviour is explained in terms of formation of a tunnel $\mathrm{n}+-\mathrm{ZnO}-\mathrm{p}-\mathrm{GaN}$ junction. 


\title{
Energy Crossovers in Nanocrystalline Zirconia
}

\author{
M.W. Pitcher, S.V. Ushakov, A. Navrotsky, B.F. Woodfield, G. Li, J. Boerio-Goates, B.M. Tissue
}

J. Am. Ceram. Soc. 88, 160, 2005

The synthesis of nanocrystalline powders of zirconia often produces the tetragonal phase, which for coarse-grained powders is stable only at high temperatures and transforms into the monoclinic form on cooling. This stability reversal has been suggested to be due to differences in the surface energies of the monoclinic and tetragonal polymorphs. In the present study, we have used high-temperature oxide melt solution calorimetry to test this hypothesis directly. Stability crossovers with increasing surface area between monoclinic, tetragonal, and amorphous zirconia were confirmed. Due to aggregation, the surface areas calculated from crystallite size are larger than those measured by BET. The fit of enthalpy versus calculated total interface/surface area gave surface enthalpies of $4.2 \mathrm{~J} / \mathrm{m}^{2}$ for the monoclinic form and $0.9 \mathrm{~J} / \mathrm{m}^{2}$ for the tetragonal polymorph. From solution calorimetry, the enthalpy of the monoclinic to tetragonal phase transition for $\mathrm{ZrO}_{2}$ was estimated to be $10 \pm 1 \mathrm{~kJ} / \mathrm{mol}$ and amorphization enthalpy to be $34 \pm 2$ $\mathrm{kJ} / \mathrm{mol}$.

\section{Synthesis of Bifunctional Polymer Nanotubes from Silicon Nanowire Templates via Atom Transfer Radical Polymerization}

\author{
M. J. Mulvihill, B. L. Rupert, R. R. He, A. Hochbaum, J. Arnold, P. D. Yang
}

J. Am. Chem. Soc. 127, 16040, 2005

As a way to control the surface properties of nanowires and nanotubes, we present a method for growing polymer from the surface of silicon/silica core/shell nanowires. After modification of nanowire surfaces with polymer initiators, Atom Transfer Radical Polymerization (ATRP) was used to grow methacrylate polymer chains from the surface. The resulting structures were characterized by SEM, TEM, and EELS. After etching the silicon cores, the resulting polymercoated nanotubes will have hydrophilic silica cores with hydrophobic polymer shells. 


\title{
Polarized Raman Confocal Microscopy of Single Gallium Nitride Nanowires
}

\author{
P. J. Pauzauskie, D. Talaga, K. Seo, P. D. Yang, F. Lagugne-Labarthet
}

J. Am. Chem. Soc. 127, 17146, 2005

Polarized Raman spectra and corresponding Raman scattering intensity images of an isolated gallium nitride nanowire with a diameter of $170 \mathrm{~nm}$ are presented. The sensitivity of the confocal microscope combined with a high-resolution piezoelectric stage enables analysis of the crystalline phase and crystallographic orientation of an individual nanowire with an excellent spatial and spectral resolution in a short acquisition time. XANES and ELNES in Ceramic Science

\section{XANES and ELNES in Ceramic Science}

\author{
I. Tanaka, T. Mizoguchi, T. Yamamoto
}

J. Am. Chem. Soc. Feature Article, 88, 2013, 2005

Both XANES and ELNES are important tools in ceramic science offering information on local environment of selected elements not only in crystals but also in amorphous materials. Recent technological progress enables measurements of XANES of ppm-level dopants using modern synchrotron facilities. Combined with transmission electron microscopy, ELNES can be used to analyze the local structures with sub-nanometer spatial resolution. First principles methods to reproduce and interpret the spectra have been established just recently. When a core-hole is adequately taken into account, most of K-edge spectra can be well reproduced using a modern band-structure method within one-electron approximation. The same is true for L2,3-edge spectra of non-transition metal compounds. However, multi-electron calculations are mandatory to reproduce L2,3-edge spectra of 3d transition-metal elements because of strong electronic correlations. In this paper, some recent results obtained in our group by the combination of XANES/ELNES experiments and theoretical calculations are reviewed. 


\title{
Rotational and Translational Diffusion of Peptide-Coated CdSe/CdS/ZnS Nanorods Studied by Fluorescence Correlation Spectroscopy
}

\author{
J. M. Tsay, S. Doose, S. Weiss
}

J. Am. Chem. Soc. 128, 1639, 2006

$\mathrm{CdSe} / \mathrm{CdS} / \mathrm{ZnS}$ nanorods (NRs) of three aspect ratios were coated with phytochelatin-related peptides and studied using fluorescence correlation spectroscopy (FCS). Theoretical predictions of the NRs' rotational diffusion contribution to the correlation curves were experimentally confirmed. We monitored rotational and translational diffusion of NRs and extracted hydrodynamic radii from the extracted diffusion constants. Translational and rotational diffusion constants (Dtrans and Drot) for NRs were in good agreement with Tirado and Garcia de la Torre's as well as with Broersma's theories when accounting for the ligand dimensions. NRs fall in the size range where rotational diffusion can be monitored with higher sensitivity than translational diffusion due to a steeper length dependence, Drot $\sim$ L-3 versus Dtrans $\sim$ L-1. Monitoring rotational diffusion of bioconjugated NRs using FCS might prove to be useful for observing binding and conformational dynamics in biological systems.

\section{Effect of GaN Template Layer Strain on the Growth of $\operatorname{In}_{\mathbf{x}} \mathrm{Ga}_{1-\mathrm{x}} \mathrm{N} / \mathrm{GaN} \mathrm{MQW}$ Light Emitting Diodes}

M.C. Johnson, E.D. Bourrett-Courchesne, J. Wu, Z. Liliental-Weber, D.N. Zakharov, R.J. Jorgenson, T.B. Ng, D.E. McCready, J. Williams

J. Appl. Phys. 96, 1381, 2004

$\mathrm{GaN}$ template layer strain effects on the growth of InGaN/GaN light emitting diodes devices were investigated. Seven-period InGaN/GaN multiple quantum well structures (MQW) were deposited on 5 and $15 \mu \mathrm{m} \mathrm{GaN}$ template layers. It was found that the electroluminescence emission of the $15 \mu \mathrm{m}$ device was redshifted by approximately $132 \mathrm{meV}$. Triple-axis x-ray diffraction and cross-sectional transmission electron microscopy show that the $15 \mu \mathrm{m}$ template layer device was virtually unstrained while the $5 \mu \mathrm{m}$ layer experienced tensile strain. Dynamic secondary ion mass spectrometry depth profiles show that the $15 \mu \mathrm{m}$ template layer device had an average indium concentration of $11 \%$ higher than that of the $5 \mu \mathrm{m}$ template layer device. It was also found that the $15 \mu \mathrm{m}$ layer device had a higher average growth rate than the $5 \mu \mathrm{m}$ template layer device. This difference indium concentration and growth rate was due to changes in thermodynamic limitations caused by strain differences in the template layers. 


\title{
Electrical, Microstructural and Thermal Stability Characteristics of $\mathrm{Ta} / \mathrm{Ti} / \mathrm{Ni} / \mathrm{Au}$ Contacts to $\mathrm{n}-\mathrm{GaN}$
}

\author{
A. Motayed, K. A. Jones, M. A. Derenge, M. C. Wood, A. V, Davydov, D. N. Zakharov, Z. \\ Liliental-Weber, S. N. Mohammed
}

J. Appl. Phys. 95, 1516, 2004

A metallization technique has been developed for obtaining low resistance Ohmic contact to $n$ $\mathrm{GaN}$. The metallization technique involves the deposition of a metal layer combination $\mathrm{Ta} / \mathrm{Ti} / \mathrm{Ni} / \mathrm{Au}$ on an $n-\mathrm{GaN}$ epilayer. It is observed that annealing at $750^{\circ} \mathrm{C}$ for $45 \mathrm{~s}$ leads to low contact resistivity. Corresponding to a doping level of $5 \times 10^{17} \mathrm{~cm}^{-3}$, the contact resistivity of the contact $\rho_{\mathrm{S}}=5.0 \times 10^{-6} \Omega \mathrm{cm} 2$. The physical mechanisms underlying the realization of low contact resistivity is investigated using current-voltage characteristics, $\mathrm{x}$-ray diffraction, Auger electron spectroscopy, transmission electron microscopy, and energy dispersive x-ray spectrometry.

\section{Investigation of Femtosecond Laser Assisted Nano and Microscale Modifications in Lithium Niobate}

D. Deshpande, A. Malshe, E. A. Stach, V. Radmilovic, D. Alexander, D. Doerr

J. Appl. Phys. 97, 74316, 1-9, 2005

A study of the physicochemical modifications at micro and nano scales as a result of femtosecond laser processing is essential to explore the viability of this process to write surface and subsurface structures in transparent media. To this end, scanning probe and transmission electron microscopy and spectroscopy techniques were used to study these modifications in lithium niobate. Evidence of a number of simultaneously occurring mechanisms such as melting, ablation, and shockwave propagation are observed in the scanning electron microscope (SEM) micrographs. X-ray diffraction (XRD), Auger and electron dispersive spectroscopy (EDS) studies indicate loss of lithium and oxygen from the immediate surface of the processed region. Raman spectroscopy analysis indicates an unchanged chemical composition in the bulk, though at a loss of crystallinity. The oblate subsurface structure contains a void at the top, indicative of localized explosive melting and rapid quenching of the affected material. 


\title{
Stable, Free-standing Ge Nanocrystals
}

I. D. Sharp, Q. Xu, C. Y. Liao, D. O. Yi, J. W. Ager III, J. W. Beeman, K. M. Yu, D. C. Chrzan. E. E. Haller

J. Appl. Phys. 97, 2005

Freestanding Ge nanocrystals that are stable under ambient conditions have been synthesized in a two-step process. First, nanocrystals with a mean diameter of $5 \mathrm{~nm}$ are grown in amorphous $\mathrm{SiO}_{2}$ by ion implantation followed by thermal annealing. The oxide matrix is then removed by selective etching in diluted HF to obtain freestanding nanocrystals on a Si wafer. After etching, nanocrystals are retained on the surface and the size distribution is not significantly altered. Freestanding nanocrystals are stable under ambient atmospheric conditions, suggesting formation of a self-limiting native oxide layer. For freestanding as opposed to embedded Ge nanocrystals, an additional amorphous-like contribution to the Raman spectrum is observed and is assigned to surface reconstruction-induced disordering of near-surface atoms.

\section{Indentation Stress Dependence of the Temperature Range of Microscopic Superelastic Behavior of Nickel-Titanium Thin Film}

\author{
Y. J. Zhang, Y. T. Cheng, D. S. Grummon
}

J. Appl. Phys. 98, 033505, 2005

The microscopic superelastic behavior of thin-film NiTi is investigated by instrumented indentation experiments conducted at different temperatures. The indentation-induced superelastic effect is found to be persistent to about $100 \mathrm{~K}$ above the austenite transformation finish temperature $\left(A_{f}\right)$. In contrast, the upper temperature where superelastic effect exists is only around $A_{f}$ plus $40 \mathrm{~K}$ in uniaxial tension and compression tests, beyond which the plasticity of the austenite phase overwhelms the transformation-induced superelasticity. By combining the Clausius-Clapeyron equation and spherical cavity model for indentation, we show that the high hydrostatic pressure under the indenter is capable of elevating the transformation temperatures and increase the upper temperature limit of indentation-induced superelastic behavior. 


\title{
Room Temperature Rectifying Characteristics of Epitaxial $\mathrm{Y}_{1} \mathrm{Ba}_{2} \mathrm{Cu}_{3-\mathrm{x}} \mathrm{Zn}_{\mathbf{x}} \mathrm{O}_{7-}$ $(x=0.0,0.2)$ and $\mathrm{Nb}: \mathrm{SrTiO}_{3}(\mathrm{Nb}: \mathbf{0 . 0 5 \%}, 0.1 \%, 0.5 \%)$ Heterojunctions
}

\author{
W. Ramadan, S. B. Ogale, S. Dhar, L. F. Fu, N. D. Browning, T. Venkatesan
}

J. Appl. Phys. 99, 04390, 2006

We report on the fabrication and electrical characterization of epitaxial metal semiconductor junctions between $\mathrm{Y}_{1} \mathrm{Ba}_{2} \mathrm{Cu}_{3-x} \mathrm{Zn}_{x} \mathrm{O}_{7-}$ (optimally doped and $\mathrm{Zn}$-doped) and (001) $\mathrm{Nb}$ : $\mathrm{SrTiO}_{3}$ (NSTO) with different $\mathrm{Nb}$ concentrations $(0.05 \%, 0.1 \%$ and $0.5 \%)$. The current-voltage characteristics of such epitaxial junctions are non-linear and rectifying and these are dramatically enhanced with decreasing $\mathrm{Nb}$ concentration and $\mathrm{Zn}$ doping. Indeed, for the case of $0.05 \%$ $\mathrm{Nb}: \mathrm{STO}$, reverse breakdown voltage as high as $-18 \mathrm{~V}(-28 \mathrm{~V})$ is realized for optimally doped ( $\mathrm{Zn}$ doped) YBCO. These data are analyzed within the framework of thermionic emission/diffusion models for Schottky and Metal-Insulator- Semiconductor (MIS) type junctions.

\section{Propagation of Surface Fatigue Cracks in Human Cortical Bone}

\author{
J. J. Kruzic, J. A. Scott, R. K. Nalla, R. O. Ritchie
}

J. Biomechanics 39, 968, 2006

An understanding of how fatigue cracks grow in bone is of importance as fatigue is thought to be the main cause of clinical stress fractures. This study presents new results on the fatigue-crack growth behavior of small surface cracks (approximately 75-1000 micron in size) in human cortical bone, and compares their growth rates with data from other published studies on the behavior of both surface cracks and many millimeter, through-thickness large cracks. Results are obtained with a cyclically loaded cantilever-beam geometry using optical microscopy to examine for crack growth after every 100-500 cycles. Based on the current and previous results, small fatigue cracks appear to become more resistant to fatigue-crack growth with crack extension, analogous to the way the fracture resistance of cortical bone increases with crack growth. Mechanistically, a theory attributing such behavior to the development of bridges in the wake of the crack with crack growth is presented. The existence of such bridges is directly confirmed using optical microscopy. 


\title{
Carbon-supported Pt-Sn Electrocatalysts for the Anodic Oxidation of H-2, $\mathrm{CO}$, and H-2/CO Mixtures. Part I. Microstructural Characterization
}

\author{
V. Radmilovic, T.J. Richardson, S.J. Chen, P.N. Ross Jr.
}

J. Catalysis 232, 199, 2005

High-resolution transmission electron microscopy (HRTEM), microchemical analysis by X-ray emission spectroscopy (EDS), and X-ray diffraction (XRD) were used to characterize the composition, size, distribution, and morphology of the phases present in three different carbonsupported Pt-Sn catalysts. The catalysts had nominal Pt/Sn atomic ratios of $1: 1$ and 3:1. The 1:1 catalyst sample heat-treated at $500^{\circ} \mathrm{C}$ contained a Pt-rich fcc alloy phase $\left(\mathrm{a}_{0}=0.3965 \mathrm{~nm}\right)$ and tetragonal $\mathrm{SnO}_{2}$. The sample from the same precursors heat treated at $900^{\circ} \mathrm{C}$ consisted of stoichiometric hexagonal PtSn, a nearly stoichiometric $\mathrm{Pt}_{3} \mathrm{Sn}$ fcc phase $\left(\mathrm{a}_{0}=0.3988 \mathrm{~nm}\right)$, and tetragonal $\mathrm{SnO}_{2}$. Neither pure tin nor pure platinum particles were detected in any of the catalysts. The $3: 1$ catalyst sample reduced in $\mathrm{H}-2$ at $270^{\circ} \mathrm{C}$ was composed entirely of the stoichiometric $\mathrm{Pt}_{3} \mathrm{Sn}$ cubic phase $\left(\mathrm{a}_{0}=0.3998 \mathrm{~nm}\right)$.

\section{Reduction of Dislocation Density in Heteroepitaxial GaN: Role of $\mathrm{SiH}_{4}$ Treatment}

\author{
K. Pakula, R. Bozek, J.M. Baranowski, J. Jasinski, Z. Liliental-Weber
}

J. Cryst. Growth 267, 1, 2004

TEM and AFM data show that a significant reduction of threading dislocations in heteroepitaxial $\mathrm{GaN} / \mathrm{Al}_{2} \mathrm{O}_{3}$ grown by MOCVD has been achieved. The reduction has been obtained by growth interruption followed by annealing in silane $\left(\mathrm{SiH}_{4}\right)$. Density of threading dislocations in the $\mathrm{GaN}$ layer above the silane-exposed surface decreased to $5 \times 10^{7} \mathrm{~cm}^{-2}$ in comparison to $10^{9} \mathrm{~cm}^{-2}$ in the layer below this surface. TEM data showed the existence of pyramidal pits at the silane-exposed surface. The presence of these pits indicates that the GaN surface was selectively etched during the silane flow. Horizontal propagation of dislocations above the surface treated by silane (where formation of $\mathrm{SiN}$ was expected) suggests that the $\mathrm{GaN}$ layer in this region was grown in the lateral epitaxial overgrowth mode. EDX measurements performed at the interface between the $\mathrm{SiH}_{4}$-treated $\mathrm{GaN}$ layer and the subsequently grown $\mathrm{GaN}$ did not show any presence of $\mathrm{Si}$. Therefore, it is believed that the dislocation reduction is related to the lateral overgrowth above the pits and not to the formation of a SiN interlayer. 


\title{
Morphological Evolution of Ge Islands on Au-Patterned Si
}

\author{
J. T. Robinson, J. A. Liddle, A. Minor, V. Radmilovic, O. D. Dubon
}

J. Cryst. Growth 287, 518, 2006

We present results on the growth of Ge islands into two-dimensional arrays by molecular beam epitaxy on Au-patterned Si. For a square array of Au dots, a two-dimensional square lattice of $\mathrm{Ge}$ islands extending over hundreds of microns has been produced. Islands in the patterned region grow between $\mathrm{Au}$ dots and have strikingly different shapes than islands on Au-free Si. For example, square base truncated pyramids grow on $\mathrm{Si}(001)$ while rod-shaped islands grow on $\mathrm{Si}(110)$. Characterization of island development on $\mathrm{Si}(001)$ shows three island shape transitions during growth with the final shape converging to that of the super-dome structure. For both $\mathrm{Si}(001)$ and $\mathrm{Si}(110)$ islands initially grow as "lens-like" structures before nucleating steep $\{111\}$ side-facets and transforming into truncated pyramids or rods, respectively.

\section{Zincblende and Wurtzite Phases in InN Epilayers and their Respective Band Transitions}

\author{
P. Specht, J. C. Ho, X. Xu, R. Armitage, E. R. Weber, E. Erni, and C. Kisielowski
}

J. Cryst. Growth 288, 225, 2006

Zincblende and wurtzite phases of $\mathrm{InN}$ are found in $\mathrm{InN}$ epilayers deposited by Molecular beam epitaxy on $\mathrm{GaN}$ buffers which were grown by metal organic chemical vapor deposition. Valence electron energy loss spectroscopy (VEELS) was applied to determine band transitions in both phases of InN. GaN buffer layers were used as VEELS reference. The chemistry and crystalline structure of the observed areas was recorded simultaneously to exclude a contribution from oxides and/or metal Clusters or extended defects Such as grain boundaries. At room temperature a band transition for wurtzite InN was found at $(1.7+/-0.2) \mathrm{eV}$ and for zincblende InN at (1.4 $+/-0.2) \mathrm{eV}$ that are ascribed to the fundamental bandgaps of the respective polytypes. Those values correlate well with recent results of various research groups measuring the bandgap in InGaN alloys with VEELS. 


\title{
Electrocatalysis by Nanoparticles - Oxygen Reduction on EBONEX/Pt Electrode
}

\author{
Lj.M. Vracar, N.V. Krstajic, V.R. Radmilovic and M.M. Jaksic
}

J. Electroanalytical Chemistry 587, 99, 2006

The oxygen reduction reaction was studied in $0.5 \mathrm{~mol} \mathrm{dm}-3 \mathrm{HClO} 4$ solution on an electrode based on Ebonex-supported platinum electrocatalyst applied on rotation Au disk electrode $(\mathrm{Au} / \mathrm{Ebonex} / \mathrm{Pt})$. Pt catalyst was prepared by impregnation method from 2-propanol solution of $\mathrm{Pt}(\mathrm{NH} 3) 2(\mathrm{NO} 2) 2$ and Ebonex powder. The synthesized catalyst was analyzed by TEM technique. The kinetics of oxygen reduction reaction on the Ebonex/Pt catalyst shows a significant enhancement in comparison with the same on polycrystalline Pt. Only a single Tafel slope of $-0.106 \mathrm{~V} \mathrm{dec}-1$ is observed and the first-rate determining step in direct four-electron reduction is proposed as a main partway in the whole range of potential. The absence of PtOH layer that inhibits oxygen reduction on polycrystalline Pt, was discussed through synergetic effect into the catalyst as a consequence of the interactions of Pt nanoparticles with Ebonex, on one hand, and, through the d-band coupling model where the surface reactivity is given by the energy shift of the surface d-states with respect to the Fermi level, on the other hand.

\section{Kinetically Controlled Formation of Nanoparticulate ZnS with Mixed Cubic and Hexagonal Stacking}

\author{
H. Zhang, B. Chen, J. F. Banfield, B. Gilbert
}

J. Mater. Chem. 16, 249, 2006

Nanoparticulate $\mathrm{ZnS}$ with mixed cubic and hexagonal close packed stacking was synthesized by reaction of zinc acetate with thioacetamide in weakly acidic solutions. The influences of temperature, reaction time, amounts of reagents and solution $\mathrm{pH}$ on the nanoparticle size and phase constitution were investigated. Experimental results suggest that the stacking in the nano$\mathrm{ZnS}$ is controlled primarily by the precipitation kinetics. Factors that slow the precipitation rate favor the growth of nanoparticles with mixed stacking, probably because the probabilities of forming wurtzite-like layers and sphalerite-like layers under these conditions are approximately equal. Under conditions of rapid precipitation, growth of sphalerite is favored, probably due to the aggregation of molecular clusters with sphalerite-like structure. New vibrational modes present in IR spectra of the nano-ZnS with mixed stacking indicate that the materials have novel optical properties. Control of defect microstructure may allow use of nano-ZnS in new technological applications. 


\title{
In Situ Transmission Electron Microscopy and Nanoindentation Studies of Phase Transformation and Pseudoelasticity of Shape Memory Titanium- Nickel Films
}

\author{
X.-G. Ma, K. Komvopoulos
}

J. Mater. Res. 20, 1808, 2005

Transmission electron microscopy (TEM) and nanoindentation, both with in-situ heating capability, and electrical resistivity measurements were used to investigate phase transformation phenomena and thermomechanical behavior of shape memory titanium-nickel (TiNi) films. The mechanisms responsible for phase transformation in the nearly equiatomic TiNi films were revealed by heating and cooling the samples inside the TEM vacuum chamber. Insight into the deformation behavior of the TiNi films was obtained from the nanoindentation response at different temperatures. A transition from elastic-plastic to pseudoelastic deformation of the martensitic TiNi films was encountered during indentation and heating. Conversely to the traditional belief, the martensitic TiNi films exhibited a pseudoelastic behavior during nanoindentation within a specific temperature range. This unexpected behavior is interpreted in terms of the evolution of martensitic variants and changes in the mobility of the twinned structures in the martensitic TiNi films, observed with the TEM during in-situ heating.

\section{Simple Constraint for Phase Retrieval with High Efficiency}

H.He

J. Opt. Soc. Am. A 23, 550, 2006

I propose the use of a simple real space constraint for iterative phase retrieval intended for diffractive imaging. The proposed constraint is a single parameter equal to the number of nonzero pixels in the image. This greatly simplifies the procedure to determine the constraint. A series of algorithms using this constraint can be easily deduced from existing algorithms, such as hybrid-input-output and difference map algorithms. The high efficiency of these algorithms is largely preserved, as confirmed by numerical studies. A concept widely used in control system theory, proportional-integral-derivative control, is shown to increase the execution speed of the proposed constraint significantly. 


\title{
Semiconductor Nanowires for Subwavelength Photonics Integration
}

\author{
D. J. Sirbuly, M. Law, H. Q. Yan, P. D. Yang
}

J. Phys. Chem. B109, 15190, 2005

This article focuses on one-dimensional (1D) semiconductor subwavelength optical elements and assesses their potential use as active and passive components in photonic devices. An updated overview of their optical properties, including spontaneous emission, ultrafast carrier dynamics, cavity resonance feedback (lasing), photodetection, and waveguiding, is provided. The ability to physically manipulate these structures on surfaces to form simple networks and assemblies is the first step toward integrating chemically synthesized nanomaterials into photonic circuitry. These high index semiconductor nanowires are capable of efficiently guiding light through liquid media, suggesting a role for such materials in microfluidics-based biosensing applications.

\section{Polarized Surface-Enhanced Raman Spectroscopy on Coupled Metallic Nanowires}

\author{
A. R. Tao, P. D. Yang
}

J. Phys. Chem. B109, 15687, 2005

Regular-shaped metal nanocrystals and their ensembles can serve as ideal substrates for studying surface-enhanced Raman scattering (SERS). We synthesized well-defined silver nanowires for a systematic study of SERS signal with respect to polarization and structural ordering. The observed dependence on polarization direction confirms prior theoretical predictions that large electromagnetic (EM) fields are localized in the interstitials between adjacent nanowires. We show that these modes are largely dipolar in nature and rely on short-range EM coupling between nanowires. 


\title{
Pt Nanocrystals: Shape Control and Langmuir-Blodgett Monolayer Formation
}

\author{
H. Song, F. Kim, S. Connor, P. Yang,
}

J. Phys. Chem. B109, 188, 2005

We report the synthesis of monodisperse Pt nanocrystals with three different shapes - cubes, cuboctahedra, and octahedra, selectively, with similar sizes of $9-10 \mathrm{~nm}$ by a modified polyol process. We found that addition of silver ion enhances the crystal growth rate along $\langle 100\rangle$, and essentially determines the shape and surface structure of the Pt nanocrystals. After the reaction, the silver species can be easily removed by repetitive precipitation giving pure Pt nanoparticles. Two-dimensional arrays of the Pt nanocrystals were assembled by using the Langmuir-Blodgett (LB) method. The particles were evenly distributed on the entire substrate, and their surface coverage and density can be precisely controlled by tuning the surface pressure. The resulting $\mathrm{Pt}$ LB layers are potential candidates for 2-D model catalysts as a result of their high surface area and the structural uniformity of the metal nanocrystals.

\section{Influence of Cation Substitution on the Magnetic Properties of $\mathrm{FeCr}_{2} \mathrm{~S}_{4}$ Ferrimagnet}

V. Tsurkan, J. R. Groza, V. Zestrea, M. Baran, R. Szymczak, H. Szymczak, M. Mucksch, F. Haider, R. Tidecks

J. Phys. Chem. Sol. 66, 2040, 2005

The influence of cation substitution on the magnetic properties of single and polycrystals of $\mathrm{FeCr}_{2} \mathrm{~S}_{4}$ spinel has been studied. The tetrahedral A-site substitution of the $\mathrm{Fe}$ by $\mathrm{Cu}$ in $\mathrm{Fe}_{1-\mathrm{x}} \mathrm{Cu}_{\mathrm{x}} \mathrm{Cr}_{2} \mathrm{~S}_{4}$ was found to increase significantly the value of temperature $\mathrm{Tm}$ of the spin-glass like magnetization anomaly, whereas the octahedral B-site substitution of the $\mathrm{Cr}$ by $\mathrm{In}$ decreases $\mathrm{Tm}$. This effect is suggested to result from a structural transformation influenced by variation of internal (chemical) pressure due to lattice contraction $(\mathrm{Cu})$ or expansion $(\mathrm{In})$. Based on the experimental data the superexchange is concluded to be the dominant exchange interaction for $0 \leq x \leq 0.5$ in $\mathrm{Fe}_{1-\mathrm{x}} \mathrm{Cu}_{\mathrm{x}} \mathrm{Cr}_{2} \mathrm{~S}_{4}$, whereas the indirect exchange through the charge carriers is considered of minor importance. 


\title{
A Systematic Study of the Oxygen K Edge in the Cubic and Less Common Monoclinic Phases of Rare Earth Oxides (Ho, Er, Tm, Yb) by Electron Energy Loss Spectroscopy
}

\author{
A. S. Harvey, B. Guo, I. M. Kennedy, S. H. Risbud, V. J. Leppert
}

J. Phys. - Condens. Matter 18, 2181, 2006

Transmission electron microscopy and electron energy loss spectroscopy (EELS) were used to distinguish structural details for the cubic and uncommon monoclinic crystal phases of heavy metal rare earth oxide nanoparticles synthesized by a combustion process. Specifically, we systematically examined the EELS oxygen $\mathrm{K}$ edge for $\mathrm{Ho}, \mathrm{Tm}, \mathrm{Er}$, and $\mathrm{Yb}$ sesquioxides in both phases. For both phases, we observed the usual double-peak structure of the oxygen $\mathrm{K}$ edge typically seen for oxygen atoms tetrahedrally surrounded by metal atoms, but we found the details of the energy loss near-edge structure to differ substantially between the two phases. Our results indicate a greater peak separation for the cubic than for the monoclinic phase. Furthermore, a trend of increasing peak separation with increasing atomic number was also noted for both phases in the series of rare earth oxide nanoparticles. Our results show that the fine structure in EELS can be utilized to determine differences on the nanoscale between the common cubic structure and the less common monoclinic structure.

\section{Tribological and Nanomechanical Properties of Unmodified and Crosslinked Ultra-High Molecular Weight Polyethylene for Total Joint Replacements}

\author{
J. Zhou, A. Chakravatula, L. Pruitt, K. Komvopoulos
}

J. Tribology 126, 386, 2004

Wear of ultra-high molecular weight polyethylene (UHMWPE) continues to be a major obstacle limiting the longevity of total joint replacements. Consequently, a systematic investigation of the initial stage of the wear process was performed in this study in order to obtain insight into the origins of wear in UHMWPE at submicrometer scales. Sliding experiments were performed with both unmodified and crosslinked (by gamma radiation treatment) UHMWPE subjected to reciprocating sliding against $\mathrm{Co}-\mathrm{Cr}$ alloy in a bath of bovine serum under ranges of mean contact pressure and sliding speed typical of knee joints. Nanoindentation and optical, scanning electron, and transmission electron microscopy were used to examine the effect of crosslinking on the nanomechanical properties, dominant wear mechanisms, and microstructure of UHMWPE. The fundamental wear micromechanisms of unmodified and crosslinked UHMWPE are interpreted in the context of coefficient of friction, wear factor, creep, adhesion force, and microstructure results. 


\title{
Wear Mechanisms of Untreated and Gamma Irradiation Ultra-High Molecular Weight Polyethylene for Total Joint Replacements
}

\author{
J. Zhou, K. Komvopoulos
}

J. Tribology 127, 273, 2005

Modification of the surface microstructure of ultra-high molecular weight polyethylene (UHMWPE) is essential for improving the wear resistance of orthopedic implants. A common approach is to cross-link the polymer by gamma irradiation. The objective of this study was to examine the tribological behaviors of untreated and gamma irradiated UHMWPE under physiologically relevant contact conditions. Transmission electron microscopy (TEM) and environmental scanning electron microscopy (ESEM) were used to examine the microstructure and morphology of the worn surfaces. Regularly spaced folds with average spacing depending on the irradiation dose (i.e. cross-link density) formed on the wear tracks. SEM and TEM results elucidated the roles of the cross-link density and crystalline lamellae in the wear process. Based on the experimental evidence, a deformation model was obtained that provides explanation for the dependence of surface folding on the cross-link density and lamellae reorientation during sliding.

\section{Classifying Terrestrial Volcanic Alteration Processes and Defining Alteration Processes They Represent on Mars}

\author{
J. L. Bishop, P. Schiffman, R. J. Southard, A. Drief, K. L. Verosub, D. J. Smith
}

Lunar Planet. Sci. XXXV. Lunar Planet. Inst. Houston, 1780, 2004

Characterizing alteration of the rocks on Mars is difficult, but some guidelines can be made through comparison with terrestrial volcanic alteration. Subaerial volcanic activity on the Hawaiian islands and subglacial volcanic activity on Iceland have led to the formation of a variety of silicate and iron oxide-rich alteration products that may serve as models for chemical alteration on Mars. Multiple samples have been collected from palagonitized tuffs, altered pillow lavas, altered tephra, and S-rich vents for study in the lab. Variations in the kinds of alteration products have been observed depending on the sample environment. We are defining associations between the alteration products and formation conditions that can be used to provide information about environmental conditions on Mars. In particular, we are providing definitions for palagonitic, pedogenic and solfataric alteration that can be used to characterize these alteration processes. 


\title{
Structure and Thermodynamics of Weakly Segregated Rod-Coil Block Copolymers
}

\author{
B. D. Olsen, R. A. Segalman
}

Macromolecules 38, 10127, 2005

The self-assembly of a series of monodisperse rod-coil block copolymers is studied in the weak segregation limit. This unusual weakly segregated system consists of polyisoprene (PI) coil blocks and poly (alkoxyphenylene vinylene) (PPV) rod blocks solubilized with alkoxy side groups. The order to microphase disorder transition (ODT) and nematic isotropic (NI) transition are experimentally investigated to produce a rod-coil block copolymer phase diagram in a system that follows polymeric scaling relationships. Small-angle X-ray scattering (SAXS), transmission electron microscopy (TEM), polarized optical microscopy, depolarized light scattering, and wide-angle X-ray scattering (WAXS) are used to map the phase diagram. As the symmetric diblock copolymer is heated, a series of transitions from lamellar to nematic to isotropic phases are observed. Transmission electron microscopy (TEM) reveals unusual grain structures in the low-temperature lamellar phase. Changes in domain spacing with temperature suggest rod rearrangements within the lamellar phase.

\section{Ultrastructural Examination of Dentin Using Focused Ion-beam Cross- sectioning and Transmission Electron Microscopy}

\author{
R.K. Nalla, A.E. Porter, C. Daraio, A. Minor, V. Radmilovic, E.A. Stach, A.P. Tomsia R.O. \\ Ritchie
}

Micron 36, 672, 2005

Focused ion-beam (FIB) milling is a commonly used technique for transmission electron microscopy (TEM) sample preparation of inorganic materials. In this study, we seek to evaluate the FIB as a TEM preparation tool for human dentin. Two particular problems involving dentin, a structural analog of bone that makes up the bulk of the human tooth, are examined. Firstly, the process of aging is studied through an investigation of the mineralization in 'transparent' dentin, which is formed naturally due to the filling up of dentinal tubules with large mineral crystals. Next, the process of fracture is examined to evaluate incipient events that occur at the collagen fiber level. For both these cases, FIB-milling was able to generate high-quality specimens that could be used for subsequent TEM examination. The changes in the mineralization suggested a simple mechanism of mineral 'dissolution and reprecipitation', while examination of the collagen revealed incipient damage in the form of voids within the collagen fibers. 


\title{
Temperature Deformation Behavior of Nanocrystalline Matrix Derived from Metallic Glass
}

\author{
N. A. Mara, A. V. Sergueeva, A. K. Mukherjee
}

Mat. Sci. Eng. A 374, 244, 2004

A single-phase $\alpha$-Fe microstructure with equiaxed, randomly oriented, $15 \mathrm{~nm}$ grains was produced through the devitrification of Vitroperm $\left(\mathrm{Fe}_{73.5} \mathrm{Cu}_{1} \mathrm{Nb}_{3} \mathrm{Si}_{15.5} \mathrm{~B}_{7}\right)$ metallic glass. This microstructure was found to be stable during tensile testing at 600DGC, showing a strain rate exponent that nominally correlates to grain boundary sliding $(\mathrm{m}=0.5)$, but with little ductility, and strengths up to $1250 \mathrm{MPa}$. It is suggested that the lack of dislocation activity at the small grain sizes can cause the observed brittle behavior. During testing at higher temperatures, a Nbcontaining phase grows at the triple points of the original microstructure, and considerable grain growth occurs. At $725 \mathrm{DGC}$, elongations as large as $65 \%$ at flow stresses of $250 \mathrm{MPa}$ were attained.

\section{Elevated Temperature Behavior of SePD Materials: Superplasticity or Enhanced Ductility?}

\author{
A. V. Sergueeva, N. A. Mara, R. Z. Valiev, A. K. Mukherjee
}

Mat. Sci. Eng. A 410, 413, 2005

The generated results on superplastic flow (SPF) in materials produced by severe plastic deformation (SePD) have been analyzed. Formation of sliding surfaces with subsequent cooperative grain boundary sliding (CGBS) has been demonstrated to be a main deformation mechanism of superplasticity under conditions of microstructural stability. A substantial role of dislocation glide (DG) in accommodation processes is shown. When grain growth is observed during deformation, continuous grain boundary migration (GBM) prevents formation of slide surfaces and CGBS is restrained. In this case, deformation of the material is more likely to occur by dislocation or diffusion creep than true SPF despite relatively large strains being reached. 


\title{
Transmission Electron Microscopy Analysis of Grain Boundary Precipitate- free-Zones (PFZs) in an AlCuSiGe Alloy
}

\author{
A. Tolley, D. Mitlin, V. Radmilovic and U. Dahmen
}

Mat. Sci. Eng. A 412, 204, 2005

We have characterized the elevated temperature $\left(190^{\circ} \mathrm{C}\right)$ precipitation sequence near the grain boundaries of an $\mathrm{AlCuSiGe}$ alloy, comparing these results to the binary $\mathrm{AlCu}$ and the ternary AlSiGe. In the quaternary alloy, there is a graded microstructure that evolves with increasing distance from the boundaries, which is generally a superposition of the precipitate-free-zones (PFZs) in the binary $\mathrm{AlCu}$ and in the ternary AlSiGe. After aging for $3 \mathrm{~h}$, this graded area consists of an approximately $140 \mathrm{~nm}$ wide region that is entirely precipitate free, followed by a $400 \mathrm{~nm}$ wide region that is denuded of Si-Ge and theta' precipitates. Rather than containing the (Si-Ge)-theta' pairs observed in the bulk, this $400 \mathrm{~nm}$ wide region contains only homogeneously nucleated theta". In the alloys, the solute depleted zones are much narrower than the total length of the PFZ. For example, in both $\mathrm{AlCu}$ and $\mathrm{AlCuSiGe}$, the $\mathrm{Cu}$ depleted zone is only $30 \mathrm{~nm}$ wide. This underscores the need for vacancies during precipitation of not only theta' and Si-Ge, but of theta" as well.

\section{Bimodal Microstructure and Deformation of Cryomilled Bulk Nanocrystalline Al-7.5Mg Alloy}

\author{
Z. Lee, D. B. Witkin, V. Radmilovic, E. J. Lavernia, S. R. Nutt
}

Mat. Sci. Eng. A 410, 462, 2005

The microstructure, mechanical properties and deformation response of bimodal structured nanocrystalline $\mathrm{Al}-7.5 \mathrm{Mg}$ alloy were investigated. Grain refinement was achieved by cryomilling of atomized Al-7.5Mg powders, and then cryomilled nanocrystalline powders blended with coarse-grained powders were consolidated by HIP followed by extrusion to produce bulk alloys. Bimodal bulk nanocrystalline $\mathrm{Al}-7.5 \mathrm{Mg}$ alloys show balanced mechanical properties of enhanced yield and ultimate strength and reasonable ductility and toughness compared to comparable conventional alloys and nanocrystalline metals. The investigation of tensile and hardness test suggests unusual deformation mechanisms and interactions between ductile coarsegrain bands and nanocrystalline regions. 


\title{
Structure-Properties Relations in Spot Friction Welded)
}

\author{
D. Mitlin, V. Radmilovic, T.-Y. Pan, Z. Feng, M. L. Santella
}

Mat. Sci. Eng. A, 2005, In Press

This work details some aspects of the microstructure-properties-processing relations in spot friction welded (also known as friction stir spot welded) $6111 \mathrm{Al}$ sheets, joined in a lap configuration. We have shown that the tool pin penetration depth has a strong effect on the failure mode of the joined samples and a lesser effect on the joint shear strength. With increasing tool pin penetration depth, and consequently with increasing depth with which the tool shoulder presses onto the top sample, the failure mode changes from brittle and concentrated near the pin hole, to ductile and away from the weld towards the base metal. The sheet interface under the tool shoulder consists four regions; a region where there is no contact at all between the two surfaces, a region where only a mechanical bond ("kissing bond") exists, a region where there is partial metallurgical bond, and a region with full metallurgical bonding. It is thus likely that recrystallization has occurred dynamically during the welding process. The material under the shoulder has a significantly larger grain size than under the pin, as well as a different texture.

\section{Role of Microstructure in the Aging-Related Deterioration of the Toughness of Human Cortical Bone}

\author{
R. K. Nalla, J. J. Kruzic, J. H. Kinney, M. Balooch, J. W. Ager III, R. O. Ritchie
}

Mat. Sci. Eng. C 2006, In Press

The aging-related deterioration of the fracture properties of bone, coupled with higher life expectancy, is responsible for increasing incidence of bone fracture in the elderly; consequently, an understanding of how these fracture properties degrade with age is essential. In this study, ex vivo fracture experiments have been performed to quantitatively assess the effect of age on human cortical bone in the proximal-distal orientation, i.e. longitudinally along the osteons. Because cortical bone exhibits rising crack-growth resistance with crack extension, the toughness is evaluated in terms of resistance-curve (R-curve) behavior, measured for bone taken from wide range of age groups (34-99 years). Using this approach, both the crack-initiation and crackgrowth toughness are determined and are found to deteriorate with age; the initiation toughness decreases some $40 \%$ over six decades from 40 to 100 years, while the growth toughness is effectively eliminated over the same age range. 


\title{
Formic Acid Oxidation on Pt/Ru Nanoparticles: Temperature Effects
}

\author{
A.V. Tripkovic, K.Dj.Popovic, J.D.Lovic, N.M. Markovic and V. Radmilovic
}

Mat. Sci. Forum 494, 223, 2005

The formic acid oxidation has been studied at various temperatures at supported $\mathrm{Pt} / \mathrm{Ru}$ catalyst in acid solution by using thin-film rotating disk method (RDE). A supported $\mathrm{Pt} / \mathrm{Ru}$ catalyst was examined by TEM, HRTEM and STM. Twinned Pt-Ru particles were observed with average particle size $4.3 \pm 0.3 \mathrm{~nm}$. At higher temperatures $(313 \mathrm{~K}, 333 \mathrm{~K})$ the reaction rate is much faster than at room temperature $(295 \mathrm{~K})$ indicating that formic acid oxidation on supported $\mathrm{Pt} / \mathrm{Ru}$ catalyst is a highly activated process. The effect of temperature on formic acid oxidation was explained by higher mobility of COad species and increased coverage and reactivity of OHad species.

\section{High-Cycle Fatigue of a Nickel-Base Superalloy ME3 at Ambient and Elevated Temperatures: Role of Grain-Boundary Engineering}

Y. Gao, M. Kumar, R. K. Nalla, R. O Ritchie

Metall. Mater. Trans. A 36, 3325, 2005

High-cycle fatigue, involving the premature initiation and/or rapid propagation of cracks to failure due to high-frequency cyclic loading, remains a principal cause of failures in gas-turbine propulsion systems. In this work, we explore the feasibility of using "grain-boundary engineering" as a means to enhance the microstructural resistance to high-cycle fatigue. Specifically, sequential thermo-mechanical processing, involving alternate cycles of strain and annealing, was used to increase the fraction of "special" grain boundaries and to break-up the interconnected network of "random" boundaries, in a commercial polycrystalline Ni-base superalloy (ME3). The effect of such grain-boundary engineering was examined on the fatiguecrack propagation behavior of large $(\sim 8-20 \mathrm{~mm})$, through-thickness cracks at $25^{\circ}, 700^{\circ}$ and $800^{\circ} \mathrm{C}$. Although there was little influence of an increased special boundary fraction at ambient temperatures, resistance to near-threshold crack growth was definitively improved at elevated temperatures, with fatigue threshold stress intensities some $10-20 \%$ higher than at $25^{\circ} \mathrm{C}$, concomitant with a lower proportion $(\sim 20 \%)$ of intergranular cracking. 


\title{
Nitrate Cancrinite: Synthesis, Characterization, and Determination of the Enthalpy of Formation
}

\author{
Q. Liu, H. Xu, A. Navrotsky
}

Microporous Mesoporous Mater. 87, 146, 2005

\begin{abstract}
Nitrate cancrinite, with an ideal formula of $\mathrm{Na}_{8}\left[\mathrm{Al}_{6} \mathrm{Si}_{6} \mathrm{O}_{24}\right]\left(\mathrm{NO}_{3}\right)_{2} 4 \mathrm{H}_{2} \mathrm{O}$, is an important constituent of high-level nuclear wastes at several US Department of Energy (DOE) storage sites. A simple synthesis route for this material is described. Pure nitrate cancrinite has been prepared using a mixed solution of sodium silicate, sodium aluminate, sodium nitrate, and sodium hydroxide. Crystallization took place rapidly at $90^{\circ} \mathrm{C}$ and the cancrinite phase emerged within a few hours of aging. Both X-ray diffraction and composition analyses indicated the formation of pure nitrate cancrinite. Solution calorimetric measurements of this material were performed with a Calvet type calorimeter operated at $701^{\circ} \mathrm{C}$ using molten lead borate $\left(2 \mathrm{PbO} \mathrm{B}_{2} \mathrm{O}_{3}\right)$ as the solvent. Enthalpies of formation from the constituent oxides and from the elements are $-903.3 \pm 15.7 \mathrm{~kJ} / \mathrm{mol}$ and $-14,258.3 \pm 17.3 \mathrm{~kJ} / \mathrm{mol}$, respectively, for a sample with the analyzed formula of $\mathrm{Na}_{7.282}\left[\mathrm{Al}_{5.854} \mathrm{Si}_{6.146} \mathrm{O}_{24}\right]\left(\mathrm{NO}_{3}\right)_{1.336}\left(\mathrm{CO}_{3}\right)_{0.046} 3.365 \mathrm{H}_{2} \mathrm{O}$.
\end{abstract}

\section{Image Based Nanocrystallography by Means of Lattice Fringe Fingerprinting and Transmission Electron Goniometry: Proof of Principle on Titania and Gold Nanoparticles}

\author{
B. Seipel, G. Upreti, R. Groebner, P. Moeck
}

Micro Nano Breakthrough Conference, 2005

Imaged-based nanocrystallography (IBN) is a well-established and general approach to deriving the crystallography of crystalline nanoparticles from high-resolution transmission electron microscope (HRTEM) images. The fringe-visibility map theory enables calculations of the probabilities to image lattice fringes for randomly oriented nanocrystals and from this "fingerprints maps as resolved in a particular TEM" that are uniquely characteristic for any nanocrystal. These maps can then be compared with experimental plots of resolved lattice spacings versus intersection angle with other resolved lattice spacings. An unknown crystal phase (of an electron microscopically pre-identified chemical stoichiometry) may then be identified out of a range of candidate structures. The fringe-visibility map theory also leads to visual models such as fringe-visibility spheres and maps that support transmission electron goniometry in the HRTEM mode. In this poster, we demonstrate IBN by means of both LFF and TEG on titania and gold nanoparticles. 


\title{
Ferromagnetic Oxides and Nitrides for Spintronics Applications
}

\author{
P. Moeck, A. Gupta, B. Seipel, L. Noice, C. Li, K. V. Rao, R. Erni, N. D. Browning, J. Guo
}

Micro Nano Breakthrough Conference, 2005

Over the last four decades, micro- and optoelectronic devices were reduced in size in order to achieve higher operation speeds and enhanced performance. As this shrinkage proceeds, the design parameters are impacted so that the materials in current use are pushed to their classical physics operation limits. Alternatives are to render the individual components multifunctional and to implement quantum mechanical operation principles such as using the spin of electrons as an additional degree of freedom. After a brief introduction to spintronics, results of a variety of structural and spectroscopic studies on ferromagnetic oxides (P. Sharma et al. Nature Materials 2 (2003) 673) and nitrides are reported. Monochromated electron energy loss spectroscopy gave insights into the band structure of such semiconductors. High-resolution phase contrast and energy dispersive X-ray spectroscopy complement the electron microscopical investigations. Quantitative powder X-ray diffraction (Rietveld) analysis and synchrotron based X-ray spectroscopy were also employed.

\section{Dynamic Behavior and Three-Dimensional Shape of Nanoscale Inclusions Embedded in a Crystalline Matrix}

\author{
T. Mizoguchi, N.D. Browning, M. B. Brawnfeld, D.A. Agard, U. Dahmen
}

Microsc. Microanal. 11, 2, 2032, 2005

The shape, size, and dynamic behavior of inclusions in solids have been studied extensively because of their effect on structural, electronic, magnetic, optical and thermodynamic properties. Very small inclusions are of particular interest because of size, shape and defect structure often lead to a new and unusual properties in the nanoscale regime. Premelting, that is, melting, below the bulk melting point, is observed in small inclusions at grain boundaries of the confining matrix. This effect was recently discovered for $\mathrm{Pb}$ particles a few tens of nanometers in size, located at special positions in a grain boundary in Al. Grain boundary inclusions adopt typical compound shapes that are faceted toward one rain and rounded toward the other grain. However, the exact site for nucleation of the phase transitions remains unknown because the shape of these inclusions is not simple. In order to understand the 3-D shape of the inclusions in a matrix, electron tomography can be used as a powerful technique. In this study, we investigate the threedimensional shape and dynamic behavior of $\mathrm{Pb}$, and $\mathrm{Pb}-\mathrm{Cd}$ inclusions in $\mathrm{Al}$. 


\title{
The TEAM Project - An Update
}

\author{
U. Dahmen
}

Microsc. Microanal. 11, 2, 2142, 2005

Due to its unique ability to characterize nanoscale structures, electron microscopy is a key tool for nanoscience. The best instruments today provide local information with a resolution of $1 \AA$ or better from individual nanostructures. Recent advances in electron optics promise a considerable improvement in the capabilities of electron microscopes, toward a new generation of electron optical instruments, which are likely to have an important impact on our understanding of the structure of materials at the atomic level.

\section{HREM and HAADF Imaging of $\mathrm{A1}_{3}(\mathrm{Sc}, \mathrm{Zr})$ Intermetallic Phase in Al Alloys}

\author{
V. Radmilovic, A. Tolley, U. Dahmen
}

Microsc. Microanal. 11, 2, 1712, 2005

Core-shell particle structures have received considerable recent attention because it has been demonstrated that the core properties can be significantly altered by the shell. The majority of research in this field has concentrated on free standing particle systems with or without ordering in two or three dimensions. Recent work on Al-Sc-Zr alloys has shown that a core-shell structure may also be responsible for the modification of properties in a precipitate-hardened alloy system. It was demonstrated that the precipitates contained $\mathrm{Sc}$ and $\mathrm{Zr}$, but that the distribution of $\mathrm{Zr}$ was not homogeneous. Instead, a $\mathrm{Zr}$ rich shell surrounded an $\mathrm{Al}_{3} \mathrm{Sc}$ core. In this report we use the compositional sensitivity of $\mathrm{Z}$-contrast imaging to show that the addition of $\mathrm{Zr}$ to an Al-Sc alloy leads to formation of a complex $\mathrm{Al}_{3}(\mathrm{Sc}, \mathrm{Zr})$ core/shell structure. 


\title{
On the Reciprocity of TEM and STEM
}

\author{
H. Rose, C. Kisielowski
}

Microsc. Microanal. 11, 2, 2114, 2005

Reciprocity is a wave optical argument first raised by Helmholtz in 1860 for optical instruments stating that ray trajectories can be inverted because of the nature of the stationary wave equation. This equation is of second in the derivative with respect to the z-coordinate. Since this coordinate is generally chosen along the optic axis, image and source points can be exchanged. In particular, the source plane in the CTEM corresponds to the detector plane in the STEM and this equivalence was pointed out in the past on numerous occasions. This talk addresses particular aspect including aberration correctors and dark field imaging.

\section{Atomic Resolution Electron Tomography on Discrete Grid: Atom Count Errors}

\author{
K. J. Batenburg, J.R. Jinschek, C. Kisielowski
}

Microsc. Microanal. 11, 2, 2120,2005

The resolution of state-of-the-art electron tomography is the nm rage. In a new approach, atomic resolution was achieved by determining the positions of all atoms in a nanocrystal from a small number of electron tomographic projections assuming that the atoms lie on a discrete grid. The exit wave is reconstructed for each of the projections and the number of atoms in each projected column is subsequently determined using channeling theory. The discrete projection data, consisting of the number of atoms in each projected column of the discrete grid, is used as input for a discrete tomography reconstruction algorithm, which computes the desired 3D reconstruction. It has been demonstrated that the 3D shape of a 309-atom nanocrystal could be reconstructed exactly from only three projections. In this case, the process of counting the number of atoms was assumed to be free of errors. However, count errors are practically realistic and occur when the number of atoms in a column is counted incorrectly, e.g. due to noise. 


\title{
Chemical Composition and Thickness Retrieval in HRTEM by a Reversed Multislice Process
}

\author{
F. R. Chen, C. Kisielowski, J. R. Jinschek, J. Plitzko, J. J. Kai
}

Microsc. Microanal. 11, 2, 2156,2005

In general terms, lattice images do not reveal the real atomic structure of the sample directly can be distorted, and information about the sample thickness and chemical composition is heavily encoded. These drawbacks relate to the presence of lens aberrations and dynamic diffraction. The development of Cs corrected TEM and software that reconstructs the complex electron exit wave function aim at removing lens aberrations. Several approaches, such as the 1s state model, reversing multislice algorithms, or simulated annealing and maximum likelihood algorithms, have been proposed to remove the dynamical scattering effect and to retrieve the crystal potential from the complex exit wave. In this paper, we present a new method to retrieve the potential map from the exit wave based on reversing multi-slice calculations.

\section{High Resolution 3-D Analysis of Quantum Dots and Other Nanostructures}

\author{
I. Arslan, T. J. V. Yates, P. A. Midgley, N. D. Browning
}

Microsc. Microanal. 11, 2, 208, 2005

While nanotechnology presents many exciting opportunities for materials science, it also presents significant challenges for characterization. These challenges arise as optimizing the functionality of nanoscale materials often rests with a precise control of the size, shape, crystal structure and composition of the material(s) being synthesized. While traditional techniques in transmission electron microscopy have provided feedback for the growth of single crystal films and polycrystalline solids (where crystal/defect structure/composition are the property determining factors), the application of these techniques to nanoscale particulates (where size and shape are important) is not as straightforward. However, the ability to characterize these features is now possible through electron tomography using Z-contrast imaging in the scanning transmission electron microscope (called STEM tomography). An example of what can be achieved with these techniques is illustrated by the analysis of Sn. 


\title{
High Spatial and Energy Resolution EELS Using a Monochromated STEM
}

\author{
N. D. Browning, R. P. Erni, J. C. Idrobo, A. Ziegler, C. F. Kisielowski, R. O. Ritchie
}

Microsc. Microanal. 11, 2, 1434, 2005

The ability to directly correlate electron energy loss spectroscopy (EELS) with Z-contrast images in the scanning transmission electron microscope (STEM) provides a powerful tool to characterize nanoscale materials. The incorporation of a monochromator into the FEI Tecnai F20 UT at the National Center for Electron Microscopy (NCEM) extends the power of these techniques by increasing the sensitivity of core-loss spectra small changes in fine-structure and by allowing the low-loss region of the spectrum to be analyzed in detail. Here, the configuration of the monochromated Tecnai will be discussed and examples of spectra obtained utilizing the highest spatial and energy resolution will be presented.

\section{Nano-scale, Ising-like Magnetic Domain Structures in $\mathrm{Fe} / \mathrm{NiO} / \mathrm{Fe}(100)$ Trilayers.}

A.K. Schmid 1, N. Rougemaille1, M. Portalupi, A. Lanzara, P. Biagioni, A. Brambilla, M.Finazzi, L. Duò

Microsc. Microanal. 11, 2, 1836, 2005

What determines the sizes and shapes magnetic domains can have at finite temperature? The question is important both fundamentally and because small/stable domains are potentially useful for data-storage and other applications. An interesting type of domain microstructures can be observed when ferromagnetic films interact with antiferromagnets. In the capping layer of $\mathrm{Fe} / \mathrm{NiO} / \mathrm{Fe}(100)$ trilayer structures, we find extremely delicate and convoluted magnetic domain patterns. The patterns include many domains that are topologically unconnected ("bubbles") and narrow elongated domains ("channels"), and the domain wall widths are much smaller than what is observed in Fe layers grown on non-magnetic substrates. In fact, the widths of some bubbleand channel-domains are smaller than the widths of just the walls of domains we observe in our $\mathrm{Fe} / \mathrm{MgO}(100)$ substrates! 


\title{
Magnetization Reorientation in Self-Organized Fe Nanowire Arrays
}

\author{
N. Rougemaille, A. K Schmid
}

Microsc. Microanal. 11, 2, 1882, 2005

Using Spin-Polarized Low-Energy Electron Microscopy (SPLEEM), we study the assembly of iron nanowires on a (110) tungsten surface and we investigate correlations between selforganization mechanisms and magnetic domain microstructures.

\section{Passive Layer Formation at $\mathrm{Pt} / \mathrm{PbTiO}_{3}$ Interfaces Identified Using STEM and EFTEM}

\author{
S. J. Welz, L. F. Fu, R. Erni, M. Kurasawa, P.C. McIntyre, N. D. Browning
}

Microsc. Microanal. 11, 2, 1746, 2005

This study shows interface reaction at the ferroelectric/electrode interface of a multi layer thin film complex oxide material, which consists of a $\mathrm{La}_{2} \mathrm{O}_{3}$-doped $\mathrm{SrTiO}_{3}$ substrate, thin films consisting subsequently of $\mathrm{PbTiO}_{3}$ and $\mathrm{Pt}$ as well as two lead oxide top layers. Particular attention has been paid on the $\mathrm{PbTiO}_{3} / \mathrm{Pt}$ interface and the compositional and electronic structure. Analytical techniques include Z-contrast imaging, EELS and EFTEM carried out on a FEI Tecnai F20 UT STEM. 


\title{
Image-based 3D Nanocrystallography by Means of Tilt Protocol/Lattice- Fringe Fingerprinting with Contemporary Side-entry Specimen Goniometers
}

\author{
P. Moeck, B. Seipel, W. Qin, E. Mandell, P. Fraundorf
}

Microsc. Microanal. 11, 2, 632, 233, 2005

The feasibility of discrete atomic resolution electron tomography by means of exit-wave reconstruction in future aberration-corrected transmission electron microscopes has recently been demonstrated for individual crystalline nanoparticles. While fully eucentric highly precise specimen goniometers will be required for that novel technique to reach its full potential, image based 3D nanocrystallography by means of tilt protocol/lattice-fringe fingerprinting can already be practiced with the current generations of TEMs and side-entry specimen goniometers. A Philips EM430 ST microscope equipped with a double-tilt goniometer that allows for $\pm 15^{\circ}$ eucentric tilt and $\pm 10^{\circ}$ non-eucentric tilt was, for example, used to determine the lattice parameters of substoichiometric $\mathrm{WC}_{1-\mathrm{x}}$ nanocrystals with the rock salt structure. Due to this relatively small tilt range, only the so called "cubic minimalistic" tilt protocol, which requires a combined tilt of $35.3^{\circ}$ about an effective $<110>$ tilt axis that coincides with the eucentric axis of the specimen goniometer, could be employed.

\section{Testing 3-D Reconstruction Methods for Electron Tomography}

\author{
S. Mehraeen, H. Stahlberg, J. E. Evans, C. Meesters, I. Arslan, P. A. Midgley, N. D. Browning
}

Microsc. Microanal. 11, 2, 336, 2005

The technique of 3-dimensional imaging known as "electron tomography", while not new in the field of biological sciences, has rarely been used for Materials Science. Electron tomography refers to any mathematical process used to reconstruct the 3-dimensional structure of a specimen from a series of 2-dimensional projections of that structure. A variety of software packages are used to perform different functions in this mathematical process. However, so far no consensus method has been determined to provide structural information in 3 dimensions with the $0.1-20$ $\mathrm{nm}$ spatial resolution required for many Materials Science applications. As developing nanostructural designs continues, interpretation of these structures merely based on 2-dimensinal electron microscopic projections of such materials and their complex overlapping morphologies becomes a less authentic practice and a routine method for reconstructing the 3-D structure of materials must be developed. 


\title{
Phase Transformation Analysis in Titanium at Nanosecond Time-Resolution Titanium at Nanosecond Time Resolution
}

\author{
A. Ziegler, H. Kleinschmidt, G. H. Campbell, N. D Browning, J.D. Colvin, O. Bostanjoglo
}

Microsc. Microanal. 11, 2, 44, 2005

There is current interest in characterizing the sequence and the dynamics of the rapid martensitic phase transformations that occur in $\mathrm{Ti}$ and its commonly used alloys during rapid heating and cooling. In the present investigation, thin polycrystalline titanium films were subjected to steep heating/cooling rates of 1011/108 Ks-1 via pulsed laser irradiation. The induced martensitic phase transformations were followed by imaging and diffraction with a high-speed transmission electron microscope on the time scale of nanoseconds. The fast processes were observed by recording bright-field electron images and selected-area diffraction patterns with an exposure time of $7 \mathrm{~ns}$ at different times after the treating laser pulse, ranging from $0 \mathrm{~ns}$ to $100 \mu \mathrm{s}$.

\section{Development of a Dynamic Transmission Electron Microscope (DTEM) for the Study of Self-Propagating Reactions in Multilayer Foils}

\author{
J.S. Kang, A. Ziegler, N.D. Browning, G.H. Campbell
}

Microsc. Microanal. 11, 2, 484, 2005

The DTEM is an analytical instrument that provides great potential for original scientific discovery because it maintains the high resolution of conventional transmission electron microscopy (TEM) but also allows scientists to explore the realm of fast time dependence (nanoseconds and faster). Often phase changes and other dynamic material events occur on such short time scales that they appear nearly instantaneous, but these occurrences could be captured for analysis if they are induced and imaged within nanoseconds, picoseconds or even femtoseconds. On this ultrafast timescale, new concepts can be discovered that correlate structure with dynamics, even finding and exploiting transient materials properties. 


\title{
Shadow FIBing - Using Geometry to Prepare TEM Samples
}

\author{
S. Welz, N. Browning, A.M. Minor
}

Microsc. Microanal. 11, 2, 834, 2005

Focused ion beam (FIB) instrumentation has proven to be extremely useful in preparing crosssectional samples for transmission electron microscopy (TEM) investigations. The two most widely used methods involve either milling a trench in a bulk material while leaving behind an electron transparent window or the so-called "lift-out" method [1]. While these two methods are very powerful in their versatility and ability to make site-specific TEM samples, they also rely on using a sacrificial layer to protect the surface of the sample as well as the removal of a relatively large amount of material, depending on the size of the initial sample. While neither of these two characteristics may be typically seen as drawbacks, some samples prohibit the use of a sacrificial layer or long milling times.

\section{Monochromated TEM/STEM and High-Resolution Electron Energy Loss Spectroscopy (HREELS) of Extraterrestrial Materials}

\author{
Z. R. Dai, J. P. Bradley G. Graham, R. Erni, N. Browning
}

Microsc. Microanal. 11, 2, 1384, 2005

A NASA-funded 200keV FEI TecnaiF20G 2 XT monochromated (scanning) transmission electron microscope has recently been installed at Lawrence Livermore National Laboratory. The microscope is equipped with a voltage-stabilized high tension (HT) supply, monochromator, high-resolution electron energy-loss spectrometer/energy filter, high-resolution annular darkfield detector, and a solid-state X-ray energy dispersive spectrometer. The high-tension tank has sections for $60 \mathrm{~Hz}$ and high frequency filtering to provide an operating voltage of $200 \mathrm{kV} \pm$ $0.005 \mathrm{~V}$. The energy filter is a Gatan model 866 Tridiem-ERS high-resolution GIF capable of $\leq 0.15 \mathrm{eV}$ energy resolution. The X-ray detector (EDAX/Genesis 4000) has a take-off angle of 20 $\mathrm{o}$, an active area of $30 \mathrm{~mm} 2$, and a solid angle of 0.3 steradians. 


\title{
Domain Structures in $\mathrm{La}_{0.8} \mathrm{Ca}_{0.2} \mathrm{CoO}_{3}$ Perovskite
}

\author{
M. Chi, N.Browning, N.Orlovskaya
}

Microsc. Microanal. 11, 2, 1464, 2005

Lanthanum cobaltites are of considerable industrial interest due to their high mixed ionic and electrical conductivities (MIEC). This material can be used as a cathode material in solid oxide fuel cells, oxygen permeable membranes and gas sensors. Domains are the dominant microstructure feature of $\mathrm{LaCoO}_{3}$ based perovskites at room temperature. They are formed by the structural transformation from a high symmetry phase at high temperature to a low symmetry phase at low temperature enable stress relieve in the material. Domain behavior relates to the mechanical properties of the materials directly. The purpose of this study is to characterize the microstructure of $\mathrm{La}_{0.8} \mathrm{Ca}_{0.2} \mathrm{CoO}_{3}$ in particular domains and domain boundaries.

\section{Application of Exit-Plane Wave Function Images in High-Resolution Transmission Electron Microscopy for Compositional Analysis of III-V Semiconductor Interfaces}

\author{
K. Mahalingam, K. G. Eyink, G. J. Brown, D. L. Dorsey, C. F. Kisielowski, A. Thust
}

Microsc. Microanal. 11, 2, 558, 2005

Existing methods based on high-resolution transmission electron microscopy (HRTEM) for compositional analysis of III-V semiconductor interfaces are applicable only to ternary systems. Furthermore, they require optimal specimen-thickness and imaging conditions, which depend on the material system under investigation. In this work we investigate the applicability of exitplane wave function (EPWF) images, retrieved from HRTEM images, for quantitative chemical analysis of II-V semiconductor interfaces. By using the focus variation technique for EPWF retrieval in combination with factorial analysis of correspondence for quantitative analysis, we show that compositional profiles along the group-III and group-V sublattices can be independently extracted. 


\title{
0.60 eV Energy Resolution in EELS Using a Depleted Thermionic LaB 6- $^{-}$ Cathode
}

\author{
C. Mitterbauer, W. Grogger, G. Kothleitner, R. Spehr, P. Tiemeijer, F. Hofer
}

Microsc. Microanal. 11, 2, 712, 2005

The overall energy resolution in Electron Energy Loss Spectroscopy (EELS) recorded in a TEM is a function of the energy spread of the initial electron beam before entering the specimen, the stability of the microscope high tension, the stability of the energy dispersive element of the spectrometer and its electronics, the local environment (mechanical vibrations, temperature and pressure fluctuations as well as parasitic magnetic stray fields), the magnitude of spectral aberrations in the spectrum plane, and the size of the object in the projector cross-over entering the spectrometer. Using a thermionic $\mathrm{LaB}_{6}$-source in a Philips CM20 (Scanning) Transmission Electron Microscope ((S) TEM), equipped with a Gatan Imaging Filter (GIF 200) and operated at $200 \mathrm{kV}$, it was ultimately possible to achieve an energy resolution of $0.60 \mathrm{eV}$ measured at the full width half maximum (FWHM) of the zero loss peak (ZLP). This ultimate resolution was only obtained with filaments after about 2000 hours of operation.

\section{Measuring Electronic Spin-Precession in Magnetic Thin Films by Spin- Polarized Low-Energy Electron Microscopy}

\author{
C. Klein, A.K. Schmid
}

Microsc. Microanal. 11, 2, 1826, 2005

Spin-precession of hot electrons during ballistic propagation through single-crystalline magnetic thin films was studied by spin-polarized low-energy electron microscopy (SPLEEM). Using SPLEEM it is possible to directly observe a spin-dependent interference pattern produced by superposition of the two branches of an electron beam, which are created by partial reflection at the film surface and partial reflection at the film-substrate interface. This electronic analog of an optical Fabry-Perot interferometer shows reflectivity maxima for certain electron energies, revealing the existence of quantum well states. The electron energies necessary for the formation of quantum interference maxima and minima are directly related to thickness and band structure of a given film and are therefore spin-dependent for magnetic materials. These effects can not only be utilized to determine the spin-resolved unoccupied electronic band structure of magnetic materials but also to study the influence of magnetic thin films on the polarization vector of an arbitrarily polarized electron beam as demonstrated in the present study. 


\title{
Self Assembled Nanostructure Networks on Surfaces of Layered Crystals
}

\author{
W. Jäger, E. Spiecker, S. Hollensteiner, F. Dietz, L. Kipp, H. Schroeder, U. Dahmen, W. Sigle
}

Microsc. Microanal. 11, 2, 200, 2005

The self-assembly of planar network nanostructures, which develop during the early stages of metal deposition on initially smooth surfaces of layered crystal compounds, may serve the development of new nanoscale materials. Using deposition of copper onto layered $\mathrm{VSe}_{2}$ crystals as model system, the metal-induced surface phenomena have been investigated combining techniques of high-resolution and analytical TEM (including spatially-resolved ELNES, STEM and EDXS measurements), SEM, and atomic force microscopy. Large nanostructures of rooflike morphology (lateral dimensions $>100 \mathrm{~nm}$ ), which consist of two strands of crystalline material, and interconnected hexagonal networks of nanostructures with smaller lateral dimensions ( several $10 \mathrm{~nm}$ ), predominantly aligned along low-index substrate directions, are observed to form on the substrate surfaces.

\section{Nano-scale, Ising-like Magnetic Domain Structures in $\mathrm{Fe} / \mathrm{NiO} / \mathrm{Fe}(100)$ Trilayers}

A.K. Schmid, N. Rougemaille, M. Portalupi, A. Lanzara, P. Biagioni, A. Brambilla, M. Finazzi, L. Duo

Microsc. Microanal. 11, 2, 1836, 2005

An interesting type of domain microstructures is observed when ferromagnetic films interact with antiferromagnets. In the capping layer of $\mathrm{Fe} / \mathrm{NiO} / \mathrm{Fe}(100)$ trilayer structure, we find extremely delicate and convoluted magnetic domain patterns. The patterns include many domains that are topologically unconnected ("bubbles") and narrow elongated domains ("channels"), and the domain wall widths are much smaller than what is observed in Fe layers grown on non-magnetic substrates. 


\title{
Synthesis and Characterization of Fe-C Core-shell Nanoparticles
}

\author{
He Li, H. Dai, R. Sinclair
}

Microsc. Microanal. 11, 1902, 2005

Encapsulating iron nanoparticles by graphitic carbon allows the existence of elemental $\mathrm{Fe}$ nanoparticles, which have high saturation magnetization at ambient conditions. In this work, Fe$\mathrm{C}$ core-shell nanoparticles are successfully synthesized. The nanoparticles are typically $5 \sim 10 \mathrm{~nm}$ in diameter. High-resolution TEM images and Fast Fourier Transforms (FFT) of selected areas reveal that the core of the nanoparticles can either be $\alpha$-Fe (BCC) or $\gamma$-Fe (FCC). Magnetic properties study indicates that these nanoparticles are superparamagnetic.

\section{TEM Study of Oxygen Precipitation in Si Wafers with Backside Layers}

\author{
R. Yu, X.F. Zhang, Q. Wang, M. Daggubati, H. Paravi
}

Microsc. Microanal, 11, 2, 2070, 2005

In the present work, we study the oxygen precipitation in arsenic doped $\mathrm{Si}$ wafers. The precipitation behavior of oxygen in two kinds of backside treatments is compared, i.e. the deposition of polycrystalline Si back layer and the damaged back surface by sand blasting. After back layer generation, the wafers were annealed at $1200^{\circ} \mathrm{C}$ for 45 minutes and $900^{\circ} \mathrm{C}$ for $15 \mathrm{hrs}$ under nitrogen ambient. TEM study revealed that the amorphous oxide precipitates formed only at the poly-Si/substrate interface. The diameter of the precipitates is about $10-20 \mathrm{~nm}$. For the $\mathrm{Si}$ wafer with the backside damaged by sand blasting, oxide precipitates formed as elongated rods extending into the Si substrate. 


\title{
In-situ Heating Electron Microscopy on $\mathrm{Cu} / \mathrm{SnO}_{2}$ Bilayer Nanoribbons
}

\author{
X.F. Zhang, M. Law, R. Yu, T. KuyKendall, P. Yang
}

Microsc. Microanal, 11, 2, 1516, 2005

In-situ electron microscopy provides a means to directly visualize interfacial processes in real time and with high spatial resolution in real and in reciprocal spaces. However, the traditional preparation of electron-transparent samples from thin films often alters the interface(s) of interest. An innovative approach is to deposit a layer of a chosen material onto a miniature substrate that is already electron-transparent and mounted for TEM imaging. $\mathrm{Cu} / \mathrm{SnO}_{2}$ bilayer nanoribbons were made by coating about $10 \mathrm{~nm}$-thick face centered cubic $\mathrm{Cu}$ layers on tetragonal single crystal $\mathrm{SnO}_{2}$ nanobelts so to study as-made, extending hetero-structure interfaces. The $\mathrm{SnO}_{2}$ ribbons typically have width and thickness ranging from $10 \mathrm{~nm}$ to $1 \mu \mathrm{m}$ and width/thickness ratios as high as ten. The thick and thin sides of a given ribbon can be either of the $\mathrm{SnO}_{2}(101)$ or (010) surfaces.

\section{Analysis of Nano-scale Strain Near Shallow Trench Isolation Structures by Energy-filtered Convergent Beam Electron Diffraction}

P. Zhang, J. Mardinly, O. Karpenko, A. Isatrov, H. He, J. Ager, C. Nelson, E. Stach, C. Kisielowski, E. Weber, J. Spence

Microsc. Microanal. 2006, In press

Shallow trench isolation (STI) is used in silicon technology for electrical isolation between devices. However, stress near the oxide-filled trenches may lead to defect generation and may affect device strain at nanometer spatial resolution and control it at nanoscale. Although the convergent beam electron diffraction (CBED) technique of TEM has been successfully applied to measure highly localized strain around STI structures, where the intertrench silicon islands have sizes larger than $200 \mathrm{~nm}$, it is not clear whether CBED can be used to characterize local strain beyond the $90 \mathrm{~nm}$ technology node. 


\title{
Analysis of Nano-scale Stress in Strained Silicon Materials and Microelectronics Devices by Energy-filtered CBED
}

\author{
P. Zhang, A. Istratov, H. He, J. Ager, C. Nelson, E. A. Stach, J. Mardinly, J.C.H. Spence, C. \\ Kisielowski, E.R. Weber
}

Microsc. Microanal. 2006, In press

The convergent beam electron diffraction (CBED) technique of transmission electron microscopy (TEM) has excellent capabilities for strain detection at high spatial resolution. Here we report on strain measurements in a bulk $\varepsilon-\mathrm{Si} / \mathrm{SiGe} / \mathrm{Si}$ wafer and a strained $35 \mathrm{~nm}$ PMOS device in which SiGe acts as the source and drain. A correlation between Raman and CBED measurements is demonstrated for relaxed $\mathrm{SiGe}$ buffer of the strained silicon material. A uniaxial compressive stress of $1.12 \mathrm{GPa}$ is determined in the channel of the PMOS device. It is also found that even in the cross-sectional TEM sample with thickness larger than $300 \mathrm{~nm}$, the intrinsic surface strain relaxation is often so severe that no recognizable high-order Laue zone lines in the CBED patterns could be collected. The amorphization of both free surfaces of the TEM sample to a range of about $80 \mathrm{~nm}$ is proposed to minimize the impact of surface strain relaxation for future study.

\section{Use of the STEM Image as a Map for Parallel-Beam Electron Diffraction from a Nano-Particle}

\author{
H.He, J.C.H. Spence
}

Microsc. Microanal. 2006, In press

It is now well known that by combining the diffraction intensity with image information in real space one can reconstruct real space images using iterative phase retrieval algorithms such as hybrid input-output (HIO). The reduced sensitivity of parallel-beam diffraction to vibration and aberrations makes the potential resolution much higher than conventional high resolution electron microscopy (HREM) using an imaging lens, where it is difficult to go beyond 1 Angstrom. This presents a new way to obtain aberration-free images on a TEM without expensive aberration correctors. 


\title{
Atomic Resolution Electron Tomography Based on Discrete Mathematics
}

\author{
J. R. Jinschek, K.J. Batenburg, H.A. Calderon, R. Kilaas, V. Radmilovic
}

Microsc. Microanal. 2006, In press

Electron tomography has become a valuable technique in materials science to investigate the three-dimensional (3-D) structure of nanomaterials. Efficient reconstruction algorithms are available, capable of making accurate tomographic reconstructions from around 100 projections of samples that are rotated in steps of 1-2 degrees to create a tomography series. By now, the spatial resolution of such methods, that commonly utilize back projection schemes for the reconstruction, reaches towards $1 \mathrm{~nm}^{3}$. Preferred imaging modes involve Z-contrast methods. These approaches usually avoid zone axis sample orientations in order to minimize non-linear intensity contributions caused by dynamic electron scattering. On the other hand it is desirable to exploit the atomic resolution capability of TEM or STEM, which benefits from dynamic scattering. However, it is impossible to record zone axis images every 1-2 degrees, because suitable zones do not occur that frequently.

\section{Multiple Sharp Bendings of Carbon Nanotubes During Growth to Produce Zigzag Morphology}

\author{
J. F. AuBuchon, L. H. Chen, A. I. Gapin, D. W. Kim, C.Daraio, S. Jin
}

Nano Lett. 4, 1781, 2004

Carbon nanotubes have been grown with a sharply defined zigzag structure by introducing changes in the direction of applied electric field during dc plasma enhanced chemical vapor deposition (PECVD). The nanotubes maintain the same diameter before and after each bend while preserving the catalyst particle at the tip of growing nanotubes. The bends have very sharp radii of curvature of only similar to $25 \mathrm{~nm}$. As a simple inclined field direction cannot produce such a zigzag growth due to the tendency of field lines intersecting perpendicular to the local surface, the bending has been introduced primarily by dramatically manipulating the electric field lines through controlled movement of field-concentrating conductor plates. 


\title{
Large-Scale Fabrication of Carbon Nanotube Probe Tips for Atomic Force Microscopy Critical Dimension Imaging Applications
}

\author{
Q. Ye, A.M. Cassell, H. Liu, K.J. Chao, J. Han, M. Meyyappan
}

Nano Lett. 4, 1301, 2004

We report an innovative approach that combines nanopatterning and nanomaterials synthesis with traditional silicon micromachining technologies for large-scale fabrication of carbon nanotube (CNT) probe tips for atomic force microscopy imaging applications. Our batch fabrication process has produced 244 CNT probe tips per 4_ wafer with control over the CNT location, diameter, length, orientation, and crystalline morphology. CNT probe tips with diameters ranging between $40-80 \mathrm{~nm}$ and lengths between 2-6 $\mu \mathrm{m}$ are found to be functional probe tips with no need for shortening. This reliable and true bottom-up wafer scale integration and fabrication process provides a new class of high performance nanoprobes. Preliminary AFM imaging results show that the CNT probe tips are strong, wear-resistant, and capable of high resolution and critical dimension imaging.

\section{Controlled Growth of Y-Junction Nanotubes Using Ti-Doped Vapor Catalyst}

\author{
N. Gothard, C. Daraio, J. Gaillard, R. Zidan, S. Jin, A. M. Rao
}

Nano Lett. 4, 213, 2004

We demonstrate a bulk process for the synthesis of Y-junction carbon nanotubes using Ti-doped Fe catalysts. It is shown that the nanotube branching can be induced or stopped at will by tuning the Ti composition in the catalyst particle that seeds the growth of nanotubes. Detailed electron microscopic studies suggest that the mechanism for the observed Y-junction formation is mediated via catalyst particle attachment on the walls of a growing MWNT, from which the branching nanotubes nucleate and grow. By controlling the Ti concentration in the precursor, cascading Y-junction series as well as quadruple junctions have been successfully synthesized, offering the possibility for interesting device applications. The simplicity and controllability of such an in-situ Y-junction fabrication technique make it an excellent source of ready-made networks for potential nanoscale devices. 


\title{
DNA Translocation in Inorganic Nanotubes
}

\author{
R. Fan, R. Karnik, M. Yue, D. Y. Li, A. Majumdar, P. D. Yang
}

Nano Lett. 5, 1633, 2005

Inorganic nanotubes were successfully integrated with microfluidic systems to create nanofluidic devices for single DNA molecule sensing. Inorganic nanotubes are unique in their high aspect ratio and exhibit translocation characteristics in which the DNA is fully stretched. Transient changes of ionic current indicate DNA translocation events. A transition from current decrease to current enhancement during translocation was observed on changing the buffer concentration, suggesting interplay between electrostatic charge and geometric blockage effects. These inorganic nanotube nanofluidic devices represent a new platform for the study of single biomolecule translocation with the potential for integration into nanofluidic circuits.

\section{Integration of Scanning Probes and Ion Beams}

\author{
A. Persaud, S. J. Park, J. A. Liddle, J. Bokor, I. W. Rangelow, T. Schenkel
}

Nano Lett. 5, 1087, 2005

We report the integration of a scanning force microscope with ion beams. The scanning probe images surface structures non-invasively and aligns the ion beam to regions of interest. The ion beam is transported through a hole in the scanning probe tip. Piezoresistive force sensors allow placement of micromachined cantilevers close to the ion beam lens. Scanning probe imaging and alignment is demonstrated in a vacuum chamber coupled to the ion beam line. Dot arrays are formed by ion implantation in resist layers on silicon samples with dot diameters limited by the hole size in the probe tips of a few hundred $\mathrm{nm}$. 


\title{
Metal-Induced Assembly of a Semiconductor Island Lattice: Ge Truncated Pyramids on Au-Patterned Si
}

\author{
J. T. Robinson, J. A. Liddle, A. Minor, V. Radmilovic, D. O. Yi, P. Alex Greaney, K. N. Long, D. \\ C. Chrzan, O. D. Dubon
}

Nano Lett. 5, 2070, 2005

We report the two-dimensional alignment of semiconductor islands using rudimentary metal patterning to control nucleation and growth. In the Ge on Si system, a square array of submicron $\mathrm{Au}$ dots on the $\mathrm{Si}(001)$ surface induces the assembly of deposited Ge adatoms into an extensive island lattice. Remarkably, these highly ordered Ge islands form between the patterned Au dots and are characterized by a unique truncated pyramidal shape. A model based on patterned diffusion barriers explains the observed ordering and establishes general criteria for the broader applicability of such a directed assembly process to quantum dot ordering.

\section{General Route to Vertical ZnO Nanowire Arrays Using Textured ZnO Seeds}

\author{
L. E. Greene, M. Law, D. H. Tan, M. Montano, J. Goldberger, G. Somorjai, P. D. Yang
}

Nano Lett. 5, 1231, 2005

A method for growing vertical $\mathrm{ZnO}$ nanowire arrays on arbitrary substrates using either gasphase or solution-phase approaches is presented. A similar to $10 \mathrm{~nm}$-thick layer of textured $\mathrm{ZnO}$ nanocrystals with their $\mathrm{c}$ axes normal to the substrate is formed by the decomposition of zinc acetate at 200-350 degrees $C$ to provide nucleation sites for vertical nanowire growth. The nanorod arrays made in solution have a rod diameter, length, density, and orientation desirable for use in ordered nanorod-polymer solar cells. 


\title{
Electrostatic Control of Ions and Molecules in Nanofluidic Transistors
}

\author{
R. Karnik, R. Fan, M. Yue, D. Y. Li, P. D. Yang, A. Majumdar
}

Nano Lett. 5, 943, 2005

We report a nanofluidic transistor based on a metal-oxide-solution (MOSol) system that is similar to a metal-oxide-semiconductor field-effect transistor (MOSFET). Using a combination of fluorescence and electrical measurements, we demonstrate that gate voltage modulates the concentration of ions and molecules in the channel and controls the ionic conductance. Our results illustrate the efficacy of field-effect control in nanofluidics, which could have broad implications on integrated nanofluidic circuits for manipulation of ions and biomolecules in subfemtoliter volumes.

\section{Multibranching Carbon Nanotubes via Self-Seeded Catalysts}

\author{
J. F. AuBuchon, L. H. Chen, C. Daraio, S. Jin
}

Nano Lett. 6, 324, 2006

A novel multibranching carbon nanotube (CNT) structure is synthesized by direct current plasma enhanced chemical vapor deposition. The structure consists of aligned CNTs, which have branches of smaller diameters growing aligned along a direction perpendicular to the original CNT. The mechanism of branching is explained in terms of a self-seeding of Ni catalyst, which is transferred by sputtering from the original catalyst particles in the backbone CNTs to the walls of those CNTs. It is also shown that the branching induced a large increase in surface area and total nanotube length and can be beneficial in supporting very fine Pt nanoparticles for fuel cell and other catalytic applications. Such an array of Y-junction nanostructures could be useful for the fabrication of a high-density array of nanoelectronics switches and transistors. 


\title{
Controlling Self-Assembled Perovskite-Spinel Nanostructures
}

H. Zheng, Q. Zhan, F. Zavaliche, M. Sherburne, F. Straub, M. P. Cruz, L. Q. Chen, U Dahmen, R. Ramesh

Nano Lett. 2006, In Press

We report a discovery that self-assembled perovskite-spinel nanostructures can be controlled simply by selecting single-crystal substrates with different orientations. In a model $\mathrm{BiFeO}_{3}$ $\mathrm{CoFe}_{2} \mathrm{O}_{4}$ system, a (001) substrate results in rectangular-shaped $\mathrm{CoFe}_{2} \mathrm{O}_{4}$ nanopillars in a $\mathrm{BiFeO} 3$ matrix; in contrast, a (111) substrate leads to triangular-shaped $\mathrm{BiFe}_{\mathrm{O} 3}$ nanopillars in a $\mathrm{CoFe}_{2} \mathrm{O}_{4}$ matrix, irrespective of the two-phase volume fraction. This dramatic reversal is attributed to the surface energy anisotropy as an intrinsic property of a crystal.

\section{Novel Method for the Electrochemical Synthesis of Nickel-Rich Oxide Nanowires}

\author{
S.A. Thorne, Y.V. Bhargava, T.S. Mintz, V. Radmilovic, Y. Suzuki, T.M. Devine
}

Nanotechnology 2, 630, 2005

Using a novel electrochemical process, functionalized and self-assembled Ni-rich oxide nanowires were grown from an Alloy $600(\mathrm{Ni}-15.5 \mathrm{a} / \mathrm{o} \mathrm{Cr}, 8 \mathrm{a} / \mathrm{o} \mathrm{Fe})$ substrate. The formation of the nanowires occurs in a high-temperature (238-288 degrees C), high-pressure (1500psi) aqueous solution by subjecting Alloy 600 to a specific progression of reducing and oxidizing conditions. The nanowires have an average diameter of $25 \mathrm{~nm}$ with a range from $3 \mathrm{~nm}$ to $100 \mathrm{~nm}$ with a maximum observed length of 20um. Energy dispersive spectra of individual nanowires have indicated a composition of $47 \mathrm{a} / \mathrm{o} \mathrm{Ni}, 4 \mathrm{a} / \mathrm{o} \mathrm{Fe}, 49 \mathrm{a} / \mathrm{o}$ O. Their composition, in conjunction with the oxidizing growth environment, suggests that the nanowires are corrosion resistant, an important property for biotechnology applications. SQUID magnetometry shows the nanowires to be ferrimagnetic, with a coercivity of approximately $800 \mathrm{e}$ and a remnant field of $0.027 \mathrm{emu} / \mathrm{g}$. Nanowires have been also synthesized on 70-30 nickel-chrome alloy. 


\title{
Metallic NEMS Components Fabricated from Nanocomposite Al-Mo Films
}

\author{
Z. Lee, C. Ophus, L.M. Fischer, N. Nelson-Fitzpatrick, K. L. Westra, S. Evoy, V. Radmilovic, U.
} Dahmen, D. Mitlin

Nanotechnology, 2006, In press.

We have fabricated fully released NEMS cantilevers of various geometries from metallic alloy nanocomposite films. At 4.3 and $20.0 \mathrm{~nm}$ thickness, these are the thinnest released metal cantilevers reported in the literature to date. Such device dimensions are very difficult to achieve using conventional metal films. We were able to overcome this limitation by using room temperature co-sputtering to synthesize nanocomposite alloy films of Al-Mo. A systematic investigation of microstructure and properties as a function of Mo content resulted in an optimum film composition of Al-32at\%Mo with a unique microstructure comprised of a dense distribution of nm-scale Mo crystallites dispersed in an amorphous Al-rich matrix. These films were found to exhibit unusually high nanoindentation hardness and a very significant reduction in roughness compared to pure $\mathrm{Al}$, while maintaining resistivity in the metallic range. A singleanchored cantilever $5 \mu \mathrm{m}$ long, $800 \mathrm{~nm}$ wide and $20 \mathrm{~nm}$ thick showed a resonance frequency of $608 \mathrm{kHz}$, yielding a Young's modulus of $112 \mathrm{GPa}$.

\section{Nanostructured Materials by High-Pressure Severe Plastic Deformation}

\author{
A.V. Sergueeva, N. A. Mara, R. Z. Valiev, A. K. Mukherjee
}

NATO ARW Proceedings, 277, 2006

This paper reviews the current understanding of the effects of microstructural characteristics on mechanical behavior of materials produced by severe plastic deformation. The elevated temperature deformation behavior of metals and intermetallics subjected to severe plastic deformation was analyzed in order to establish possible deformation mechanisms and microstructural effects. 


\title{
Spontaneous Formation of Nanoparticle Stripe Patterns Through Dewetting
}

\author{
J. X. Huang, F. Kim, A. R. Tao, S. Connor, P. D. Yang
}

Nature Mater. 4, 896, 2005

Significant advancement has been made in nanoparticle research, with synthetic techniques extending over a wide range of materials with good control over particle size and shape (1-6). A grand challenge is assembling and positioning the nanoparticles in desired locations to construct complex, higher-order functional structures. Controlled positioning of nanoparticles has been achieved in pre-defined templates fabricated by top-down approaches $(7,8)$. A self-assembly method, however, is highly desirable because of its simplicity and compatibility with heterogeneous integration processes. Here we report on the spontaneous formation of ordered gold and silver nanoparticle stripe patterns on dewetting a dilute film of polymer-coated nanoparticles coating on a water surface. Well-aligned stripe patterns with tunable orientation, thickness and periodicity at the micrometer scale were obtained by transferring nanoparticles from a coating film onto a substrate in a dip-coating fashion.

\section{Nanowire Dye-sensitized Solar Cells}

\author{
M. Law, L. E. Greene. J. C. Johnson, R. Saykally, P. D. Yang
}

Nature Mater. 4, 455, 2005

Excitonic solar cells -including organic, hybrid organic inorganic and dye-sensitized cells (DSCs)-are promising devices for inexpensive, large-scale solar energy conversion. The DSC is currently the most efficient and stable excitonic photocell. Central to this device is a thick nanoparticle film that provides a large surface area for the adsorption of light-harvesting molecules. However, nanoparticle DSCs rely on trap-limited diffusion for electron transport, a slow mechanism that can limit device efficiency, especially at longer wavelengths. Here we introduce a version of the dye-sensitized cell in which the traditional nanoparticle film is replaced by a dense array of oriented, crystalline $\mathrm{ZnO}$ nanowires. The nanowire anode is synthesized by mild aqueous chemistry and features a surface area up to one-fifth as large as a nanoparticle cell. The direct electrical pathways provided by the nanowires ensure the rapid collection of carriers generated throughout the device, and a full Sun efficiency of $1.5 \%$ is demonstrated, limited primarily by the surface area of the nanowire array. 


\title{
Novel Electrical Switching Behavior and Logic in Carbon Nanotube Y- Junctions
}

\author{
P. R. Bandaru, C. Daraio, S. Jin, A. M. Rao
}

Nature Mater. 4, 663, 2005

Carbon-nanotube-based electronics offers significant potential as a nanoscale alternative to silicon-based devices for molecular electronics technologies. Here, we show evidence for a dramatic electrical switching behavior in a Y-junction carbon-nanotube morphology. We observe an abrupt modulation of the current from an on- to an off-state, presumably mediated by defects and the topology of the junction. The mutual interaction of the electron currents in the three branches of the Y-junction is shown to be the basis for a potentially new logic device. This is the first time that such switching and logic functionalities have been experimentally demonstrated in Y-junction nanotubes without the need for an external gate. A class of nanoelectronic architecture and functionality, which extends well beyond conventional field-effect transistor technologies, is now possible.

\section{Optical Trapping and Integration of Semiconductor Nanowire Assemblies in Water}

\author{
P. J. Pauzauskie, A. Radenovic, E. Trepagnier, H. Shroff, P. D. Yang, J. Liphardt
}

Nature Mater. 5, 97, 2006

Semiconductor nanowires have received much attention owing to their potential use as building blocks of miniaturized electrical, nanofluidic and optical devices. Although chemical nanowire synthesis procedures have matured and now yield nanowires with specific compositions and growth directions, the use of these materials in scientific, biomedical and microelectronic applications is greatly restricted owing to a lack of methods to assemble nanowires into complex heterostructures with high spatial and angular precision. We show that an infrared single-beam optical trap can be used to trap, transfer and assemble high-aspect-ratio semiconductor nanowires into arbitrary structures in a fluid environment. Nanowires with diameters as small as $20 \mathrm{~nm}$ and aspect ratios of more than 100 can be trapped and transported in three dimensions, enabling the construction of nanowire architectures that may function as active photonic devices. Moreover, nanowire structures can now be assembled in physiological environments, offering new forms of chemical, mechanical and optical stimulation of living cells. 


\title{
X-ray Microdiffraction Characterization of Deformation Heterogeneities in BCC Crystals
}

\author{
K.R. Magid, E.T. Lilleodden, N. Tamura, J.N. Florando, D.H. Lassila, M.M. LeBlanc, R.I. \\ Barabash, J.W. Morris Jr.
}

Neutron and X-Ray Scattering as Probes of Multiscale Phenomena, 840, 2005

The deformation behavior of BCC metals is being investigated by $\mathrm{x}$-ray microdiffraction measurements $(\mu \mathrm{XRD})$ for the purpose of characterizing the dislocation structure that results from uniaxial compression experiments. The high brilliance synchrotron source at the Advanced Light Source (Lawrence Berkeley National Lab) and the micron resolution of the focusing optics allow for the mapping of Laue diffraction patterns across a sample. An important finding is the observation of Laue spot "streaking", which indicates localized rotations in the lattice. Theoretical modeling of the diffraction response for various slip systems is presented, and compared to experimental data. Preliminary results include orientation maps from a highly strained Ta bicrystal and a less highly strained Mo single crystal. The results suggest that $\mu$ XRD may be a particularly useful tool for microscale studies of deformation patterns in a multi-scale investigation of the mechanisms of deformation that ranges from macroscopic deformation tests to high resolution TEM studies of dislocation structures.

\section{Analytical TEM Investigations on Boron Carbonitride Nanotubes Grown via Chemical Vapor Deposition}

\author{
R M Wang, H. Z. Zhang
}

New J. Phys. 6, 1, 2004

A systematic microstructure investigation on the boron carbonitride $(\mathrm{BCN})$ nanotubes, synthesized by bias-assisted hot-filament chemical vapor deposition, is reported. The BCN nanotubes were found to be well-crystallized with uniform diameters and transverse connections inside. Their lengths can be over a few tens of micrometers. Transmission electron microscopy (TEM) analyses indicate that the BCN nanotubes have near zigzag graphic-layered structures with helical angles of less than $30^{\circ}$. Spatial-resolved electron energy loss spectroscopy (EELS) demonstrates typical $\mathrm{sp}^{2}$ bonding configuration of the nanotubes with pronounced characteristic $\pi$ and _ peaks of boron and carbon and characteristic K-shell ionization edges of nitrogen. EELS and energy-filtered TEM analyses reveal that the inner layer of the nanotube contains mass of carbon and some amount of boron, and the outer layer and the connections across the inner walls are rich in boron. The nitrogen is found distributing in the nanotube dispersedly and traceable amount of oxygen is measured existing in the shell of the nanotube. 


\title{
Image-Based Nanocrystallography by Means of Transmission Electron Goniometry
}

\author{
P. Moeck, W. Qin, P. Fraundorf
}

Nonlinear Analysis 63, 1323, 2005

Since the crystallographic phase and morphology of many materials changes with the crystal size in the 1-100 $\mathrm{nm}$ range and the potential technological applications of nanoparticles are enormous, a need arises to determine the crystallography of nanoparticles individually. Direct space high-resolution phase-contrast transmission electron microscopy (TEM) and atomic resolution Z-contrast scanning TEM when combined with goniometry of direct and/or reciprocal lattice vectors offer the possibility of developing dedicated nanocrystallography characterization methods for such small nanoparticles. Although experimentally feasible for cubic nanocrystals with lattice constants larger than $0.4 \mathrm{~nm}$ in contemporary high-resolution TEMs with modest tilt range, image-based nanocrystallography by means of transmission electron goniometry has so far only been employed by a few specialists worldwide. This is likely to change in the future with the availability of aberration-corrected TEMs.

\section{TEM and PL Characterization of Erbium and Oxygen Co-Implanted LT- GaAs:Be}

\author{
R. L. Maltez, E. Riberio, A. A. Bernussi, L. Amaral, M. Behar, P. Specht, Z. Liliental-Weber
}

Nuclear Instruments and Methods in Physics Research B 218, 450, 2004

Er and $\mathrm{O}$ have been co-implanted into Be doped low-temperature LT-GaAs. Plateau-like profiles from 10 to $\sim 145 \mathrm{~nm}$ below the sample surface and with an Er concentration at $10^{19} \mathrm{~cm}^{-3}$ were obtained. Samples with different Er to $O$ ratios (1:0, 1:2, 1:4 and 1:8) were prepared and annealed in the $550-850{ }^{\circ} \mathrm{C}$ temperature range for $30 \mathrm{~s}$. Photoluminescence (PL) data for samples with 1:2 ratio shows that Er intensity is enhanced by a factor of $\sim 5$ as compared to the non-oxygen implanted samples. In order to optically activate Er higher temperatures are required compared to the samples where oxygen is not introduced. Once Er emission is activated, it is found to be thermally stable. Transmission electron microscopy (TEM) shows no Er-rich precipitation for the co-implanted samples while those samples without oxygen have ErAs precipitates. We believe that the absence of these precipitates in the co-implanted samples is responsible for better temperature stability for Er emission. 


\title{
Anisotropic Thermal Properties of Single-Wall-Carbon-Nanotube-Reinforced Nanoceramics
}

\author{
G. D. Zhan, J. D. Kuntz, H. Wang, C. M. Wang, A. K Mukherjee
}

Philos. Mag. Lett. 84, 419, 2004

Dense single-wall carbon-nanotube (SWCNT) reinforced alumina nanocomposites have been fabricated by a novel spark-plasma-sintering technique. Anisotropic thermal properties have been found in carbon nanotube composites. The introduction of ropes of SWCNTs gives rise to a decrease in the transverse thermal diffusivity with increasing carbon nanotube content while it does not change the in-plane thermal diffusivity. This is scientifically interesting and technologically important for the development of materials for novel thermal barrier coatings.

\section{Electron Density and Implication for Bonding in $\mathrm{Cu}$}

\author{
B. Jiang, J. Friis, R. Holmstad, J. Zuo, M.O'Keeffe, J. C. H. Spence
}

Phys. Rev. B 69, 245110, 2004

We report highly accurate measurements for the low-order Fourier components of the crystal potential in copper. These were obtained by transmission electron diffraction using a small probe and multiple scattering analysis. They were used to refine the $\mathrm{Cu} 3 \mathrm{~d}$ radial wave function. An accurate charge-deformation map and a $3 \mathrm{~d}$ orbital radial wave function were obtained by using a multipole refinement of the structure factors obtained from combined quantitative electron diffraction and g-ray diffraction measurements. The results show a large change in the $3 \mathrm{~d}$ orbital radial function from d-band formation and $d-s$ band crossing ( $d-s$ hybridization). Band theory calculations are in excellent agreement with the measurements and show that the charge deformation in $\mathrm{Ag}$ is very similar to that in $\mathrm{Cu}$. Our findings are in general agreement with the monovalent description of these metals. 


\title{
Vacancy-Mediated Mechanism of Nitrogen Substitution in Carbon Nanotubes
}

\author{
D. Srivastava, M. Menon, C. Daraio, S. Jin, B. Sadanadan, A. M. Rao
}

Phys. Rev. B 69, 153414, 2004

Nitrogen substitution reaction in a graphene sheet and carbon nanotubes of different diameter are investigated using the generalized tight-binding molecular dynamics method. The formation of a vacancy in curved graphene sheet or a carbon nanotube is found to cause a curvature dependent local reconstruction of the surface. Our simulations and analysis show that vacancy mediated $\mathrm{N}$ substitution (rather than $\mathrm{N}$ chemisorption) is favored on the surface of nanotubes with diameter larger than $8 \mathrm{~nm}$. This predicted value of the critical minimum diameter for $\mathrm{N}$ incorporation is confirmed by experimental results presented on nitrogen-doped multiwalled nanotubes with approximate to 5 at. \% nitrogen prepared by the thermal chemical vapor deposition process.

\section{Electrical Properties of Epitaxial Junctions Between $\mathrm{Nb}_{\mathrm{SrTiO}_{3}}$ and Optimally Doped, Underdoped, and $\mathrm{Zn}$-Doped $\mathrm{YBa}_{2} \mathrm{Cu}_{3} \mathrm{O}_{7}$}

W. Ramadan, S. B. Ogale, S. Dhar, L.F. Fu, S. R. Shinde, D. C. Kundaliya, M.S.R. Rao, N.D. Browning, T. Venkatesan

Phys. Rev. B 72, 205333, 2005

Epitaxial thin films of optimally doped, underdoped and $\mathrm{Zn}$-doped $\mathrm{YBa}_{2} \mathrm{Cu}_{3} \mathrm{O}_{7 \text {-delta }}$ were grown on single crystal (001) $\mathrm{Nb}: \mathrm{SrTiO}_{3}$ substrates by pulsed laser deposition (PLD) and the electrical properties of the corresponding interface junctions were examined. The films/heterointerfaces were characterized by x-ray diffraction, Rutherford backscattering channeling (RBSC) spectrometry in normal and oxygen resonance modes, magnetic susceptibility, four probe inplane resistivity, and the temperature dependent junction current-voltage (I-V) characteristics. Non-linear I-V curves (forward and reverse) were obtained in all the cases, revealing some characteristic differences and interesting temperature evolution. These data were analyzed within the framework of a standard description of the transport across the metal-semiconductor (Schottky) interface and the curve fitting parameters were extracted in each case. An attempt is made to relate the observed parametric differences to the existing knowledge about the normal state and superconducting properties of the films. 


\title{
Evidence Against a Charge-density Wave on Bi (111)
}

T.K. Kim, J. Wells, C. Kirkegaard, Z. Li, S. Hoffman, J. Gayone, I. Fernaqndez-Torrente, P. Haberle, J. Pascual, K. Moore, A. Schwartz, H. He, J. Spence, K. Downing, S. Lazar, F. Tichelear, S. Borisoe

Phys. Rev. B, 72, 085440, 2005

The Bi (111) surface was studied by scanning tunneling microscopy (STM), transmission electron microscopy (TEM), and angle-resolved photoemission spectroscopy (ARPES) in order to verify the existence of a recently proposed surface charge-density wave (CDW) (Ch. R. Ast and H. Hohst, Phys. Rev. Lett. 90, 016403 (2003)). The STM and TEM results do not support a CDW scenario at low temperatures. Furthermore, the quasiparticle interference pattern observed in STM confirms the spin-orbit split character of the surface states which prevents the formation of a CDW, even in the case of good nesting. The dispersion of the electronic states observed with ARPES agrees well with earlier findings. In particular, the Fermi contour of the electron pocket at the center of the surface Brillouin zone is found to have a hexagonal shape. However, no gap opening or other signatures of a CDW phase transition can be found in the temperaturedependent data.

\section{Mapping the Spin-Dependent Electron Reflectivity of $\mathrm{Fe}$ and $\mathrm{Co}$ Ferromagnetic Thin Films}

\author{
J. Graf, C. Jozwiak, A. K. Schmid, Z. Hussain, A. Lanzara
}

Phys. Rev. B 71, 144429, 2005

Spin Polarized Low Energy Electron Microscopy is used as a spin dependent spectroscopic probe to study the spin dependent specular reflection of a polarized electron beam from two different magnetic thin film systems: Fe/W (110) and Co/W(110). The reflectivity and spindependent exchange-scattering asymmetry are studied as a function of electron kinetic energy and film thickness, as well as the time dependence. The largest value of the figure of merit for spin polarimetry is observed for a 5 monolayer thick film of $\mathrm{Co} / \mathrm{W}(110)$ at an electron kinetic energy of $2 \mathrm{eV}$. This value is 2 orders of magnitude higher than previously obtained with state of the art Mini-Mott polarimeter. We discuss implications of our results for the development of an electron-spin-polarimeter using the exchange-interaction at low energy. 


\title{
Photoluminescence Band of Hf Origin in Hafnium-Implanted Silicon in the Energy Range $700 \mathrm{meV}$ to $950 \mathrm{meV}$
}

\author{
R. Sachdeva, A. A. Istratov, P. N. K. Deenapanray, E. R. Weber
}

Phys. Rev. B-Condensed Matter, 71, 195208, 2005

\begin{abstract}
A new photoluminescence (PL) band in the energy range of $700 \mathrm{meV}$ to $950 \mathrm{meV}$ associated with hafnium implanted in silicon is reported. A shift in the position of photoluminescence peaks observed on the samples implanted with two different isotopes of Hf confirms the Hf-related origin of the observed photoluminescence band. Activation of the Hf-optical centers requires a $1000^{\circ} \mathrm{C}$ anneal step. The spectrum consists of five peaks in the rapidly quenched sample as opposed to twenty-one in the slowly cooled sample. Temperature and excitation power dependent PL measurements were performed to identify their nature. The $943.8 \mathrm{meV}$ line was associated with an exciton complex, while the $896.6 \mathrm{meV}$ line originates from impurity bound exciton. The $896.6 \mathrm{meV}$ emission line appears to be related to a Hf-related deep level defect at EC- $0.22 \mathrm{eV}$, the $845 \mathrm{meV}$ line to a deep level defect at EC- $0.27 \mathrm{eV}$. The pressure measurements indicate that the $845 \mathrm{meV}$ peak could be an internal transition. It is also found that oxygen coimplantation enhances the PL intensity in rapidly quenched samples.
\end{abstract}

\section{Spin-Induced Forbidden Evanescent States in III-V Semiconductors}

\author{
N. Rougemaille, H. J. Drouhin, S. Richard, G. Fishman, A.K. Schmid
}

Phys. Rev. Lett. 95, 186406, 2005

Within the band gap of a semiconductor no electronic propagating states are allowed, but there exist evanescent states, which govern charge transport such as tunneling. In this work, we address the issue of their spin dependence in III-V semiconductors. Taking into account the spinorbit interaction, we treat the problem using a k.p 14/14 Hamiltonian that we numerically compute for GaAs. Our results show that the removed spin degeneracy in the band gap can lead to incredibly high energy splittings and induces forbidden zones in $\mathrm{k}$ space where no evanescent states exist. 


\title{
Phase Measurement for Accurate Mapping of Chemical Bonds
}

\author{
M. Spackman, B. Jiang, T. Groy, H. He, A.E. Whitten, J.C.H. Spence
}

Phys. Rev. Lett. 95, 085502, 2005

\begin{abstract}
Although the electron density is fundamental to the study of chemical bonding and density functional theory, it cannot be accurately mapped experimentally for the important class of crystals lacking inversion symmetry, since structure factor phase information is normally inaccessible. We report the combination of X-ray and electron diffraction experiments for the determination of the electron density in acentric AlN, using multiple scattering effects in convergent-beam electron diffraction to obtain sensitivity to structure factor phases, and describe a new error metric and weighting scheme for multipole refinement using combined measurements of structure factor magnitudes and phases.
\end{abstract}

\section{Spin-Dependent Fabry-Pérot Interference from a Cu Thin Film Grown on fcc Co (001)}

\author{
Y. Z. Wu, A. K. Schmid, M. S. Altman, X. F. Jin, Z. Q. Qiu
}

Phys. Rev. Lett. 94, 027201, 2005

Spin-dependent electron reflection from a $\mathrm{Cu}$ thin film grown on $\mathrm{Co} / \mathrm{Cu}(001)$ was investigated using Spin Polarized Low Energy Electron Microscopy (SPLEEM). Fabry-Pérot type interference was observed and is explained using the phase accumulation model. SPLEEM images of the $\mathrm{Cu}$ overlayer reveal magnetic domains in the Co underlayer, with the domain contrast oscillating with electron energy and $\mathrm{Cu}$ film thickness. This behavior is attributed to the spin-dependent electron reflectivity at the $\mathrm{Cu} / \mathrm{Co}$ interface, which leads to spin-dependent FabryPérot electron interference in the $\mathrm{Cu}$ film. 


\title{
Polarity Switching and Transient Responses in Single Nanotube Nanofluidic Transistors
}

\author{
R. Fan, M. Yue, R. Karnik, A. Majumdar, P. D. Yang
}

Phys. Rev. Lett. 95, 086607, 2005

We report the integration of inorganic nanotubes into metal-oxide-solution field effect transistors (FETs), which exhibit rapid field effect modulation of ionic conductance. Surface functionalization, analogous to doping in semiconductors, can switch the nanofluidic transistors from p-type to ambipolar and n-type field effect transistors. Transient study reveals the kinetics of field effect modulation is controlled by ion-exchange step. Nanofluidic FETs have potential implications in subfemtoliter analytical technology and large-scale nanofluidic integration.

\section{Experimental Study of Internal Gettering Efficiency of Iron in Silicon}

\author{
A. Haarahiltunen, H. Vainola, M. Yli-Koski, E. Saarnilehto, J. Sinkkonen
}

Physica Scripta 114, 91, 2004

We have studied internal gettering efficiency of iron in silicon by Deep Level Transient Spectroscopy DLTS and standard lifetime - methods (SPV, $\mu$ PCD). Conventional highlowhigh anneals were performed to produce a series of wafers with varying denuded zone DZ width and oxygen precipitation density. The wafers were intentionally iron contaminated to a level of about $3-510^{13} \mathrm{~cm}^{-3}$. After contamination the wafers were annealed at $900 \mathrm{C}$ and then slowly cooled to $850,800,750,700$ or $600 \mathrm{C}$. After cooling the remaining interstitial iron concentration was measured by SPV, $\mu$-PCD and DLTS. The experimental results are compared with simulations. Our results indicate that with this contamination level, the gettering is effective only at temperatures below $750 \mathrm{C}$ when iron is supersaturated over a factor of twenty. For temperatures above $750 \mathrm{C}$ the gettering is limited by iron precipitation in the bulk. 


\section{MOCVD Growth of InGaN: Mg for GaN/InGaN HBTs}

T. Chung, J.B. Limb, U. Chowdhury, P. Li, J.H. Ryou, D. Yoo, D. Zakharov, Z. Liliental-Weber, R.D. Dupuis

Physica Status Solidi 2, 2157, 2005

InGaN:Mg growth by metalorganic chemical vapor deposition (MOCVD) for GaN/InGaN HBTs is studied. Atomic Force Microscope (AFM) images show that the surface roughness is reduced by increasing the growth temperature and decreasing the growth pressure. In addition, the indium mole fraction of the InGaN layers can be controlled by varying the growth rate - a faster growth rate giving a higher indium composition. Hall measurements show that a hole concentration of 4 - $10^{18} \mathrm{~cm}^{-3}$ can be consistently produced with a $\mathrm{Cp}_{2} \mathrm{Mg}$ molar flow rate in the range of 0.18 $\mu \mathrm{m} / \mathrm{min}$ at a growth temperature of $800{ }^{\circ} \mathrm{C}$. GaN/InGaN HBT structures are grown on sapphire substrates and the Secondary Ion Mass Spectroscopy (SIMS) profiles indicate abrupt doping profiles with no $\mathrm{Mg}$ memory effect or evidence of $\mathrm{Mg}$ diffusion from the InGaN:Mg base layer into the emitter.

\section{Quantitative Electron Microscopy of InN-GaN Alloys}

Physica Status Solidi A 203, 3, 2006

T. Bartel, J. R. Jinschek, B. Freitag, P. Specht, C. Kisielowski

The local element distribution in quantum wells largely affects physical properties of devices made from such materials. In the past, quantitative electron microscopy was developed to access the stoichiometry on an atomic scale as shown on the cover page of this issue's Editor's Choice for the $\mathrm{GaN} / \mathrm{In}_{\mathrm{x}} \mathrm{Ga}_{1-\mathrm{x}} \mathrm{N} / \mathrm{GaN}$ and the $\mathrm{GaAs} / \mathrm{Al}_{\mathrm{x}} \mathrm{Ga}_{1-\mathrm{x}} \mathrm{As} / \mathrm{GaAs}$ system by the application of QUANTITEM and Chemical Imaging, respectively. In case of $\mathrm{GaN} / \mathrm{In}_{\mathrm{x}} \mathrm{Ga}_{1-\mathrm{x}} \mathrm{N} / \mathrm{GaN}$ local strain mapping allows for extracting similar data and an unusual large indium fluctuation can be observed if compared with the aluminum distribution in GaAs/AlxGa1-xAs/GaAs quantum well structures. However, radiation damage, sample preparation and microscope stability affect the data analyses and it is of essence to monitor and control such effects as outlined in the related paper. 


\title{
Quantitative Electron Microscopy of InN-GaN Alloys
}

\author{
T. Bartel, J. R. Jinschek, B. Freitag, P. Specht, and C. Kisielowski
}

Physica Status Solidi A 203, 167, 2006

Time series of high-resolution lattice images are examined to probe for possible alterations of the indium distribution in $\mathrm{GaN} / \mathrm{InxGa1-xN/GaN}$ quantum well structures during electron irradiation with energies of $150 \mathrm{kV}$ and $800 \mathrm{~W}$. By comparison with theory it is reasoned that sample preparation, microscope stability, and chosen acceleration voltages are essential factors that determine the reliability of the results. If considered, it is shown that for relevant time scales of $<$ 2 minutes and current densities of $20-40 \mathrm{~A} / \mathrm{cm}(2)$ no measurable alteration of the initial element distribution occurs. A quantitative method is highlighted for the characterization of existing indium fluctuations that are concentration dependent. Consistency between measurements from lattice images, Z-contrast images, and local band gap measurements support our conclusion. It is argued that the formation of such indium clusters is driven by strain-dependent spinodal decomposition.

\section{Classic and Novel Methods of Dislocation Reduction in Heteroepitaxial Nitride Layers}

\section{J. Jasinski}

Physica Status Solidi C 2, 994, 2005

Due to the lack of commercially available large size native substrates, reduction of threading dislocations remains one of the main challenges of group III-nitride based technology. This paper reviews major methods aimed at producing high-quality heteroepitaxial nitride films with reduced densities of dislocations. Methods such as, incorporation of buffer layers, substrate nitridation, interlayer insertion, silane-ammonia treatment, lateral overgrowth and growth of thick nitride layers are reviewed. Advantages and drawbacks of these methods are discussed and compared. 


\title{
Spontaneous Superlattice Formation in MOVPE Growth of AIGaN
}

\author{
K. Pakula, R. Bozek, J. M. Baranowski, J. Jasinski
}

Physica Status Solidi C 2, 1073, 2005

It has been found that $\mathrm{AlGaN}$ layers grown by MOCVD on sapphire substrates form superlattice (SL) along growth direction. Transmission electron microscopy (TEM) and atomic force microscopy (AFM) found the phenomenon. The amount of $\mathrm{Al}$ in $\mathrm{AlGaN}$ layer oscillates between $10 \%$ and $20 \%$ The period of oscillations is close to $30 \mathrm{~nm}$. TEM and AFM reveal a homogenous lateral distribution of the SL. The vertical extension covers the total layer thickness. It has been found that the appearance of SL depends on the state of the buffer layer on which the growth of $\mathrm{AlGaN}$ layer is initiated.

\section{Growth and Characterization of Epitaxial GaN Thin Films on 4H-SiC (1120) Substrates}

\author{
B. P. Wagner, Z. J. Reitmeier, E. A, Preble, D. N. Zakharov, Z. Liliental-Weber, R. F. Davis
}

Proc. MRS 798, 375, 2004

$\mathrm{GaN}$ thin films were grown via metalorganic vapor phase epitaxy on a-plane $4 \mathrm{H}-\mathrm{SiC}(11.0)$ substrates using an AlN buffer layer. Atomic force microscopy images show that the microstructure of the AlN buffer layer and the subsequently deposited GaN have a highly oriented growth structure where parallel growth features propagate in the [1-1.0] direction. No surface features were resolved using scanning electron microscopy imaging and the interfaces between the substrate, buffer layer, and epi-layer were continuous. Cracking was observed for an undetermined $\mathrm{GaN}$ thickness greater than $800 \mathrm{~nm}$. Plan-view transmission electron microscopy analysis showed stacking fault and threading dislocation densities to be $\sim 1.6 \times 106 \mathrm{~cm}-1$ and $\sim$ $3.3 \times 1010 \mathrm{~cm}-1$, respectively. X-ray diffraction confirmed that the $\mathrm{GaN}$ deposited epitaxially in the same orientation as the substrate. The average on-axis $\mathrm{x}$-ray full-width half-maximum of the (11.0) reflection is 948 arcsec. The average off-axis full-width half-maximum for the (10.0) reflection is 5448 arcsec. 


\title{
Microstructure of Nonpolar a-Plane GaN Grown on (1120) 4H-SiC
}

\author{
D. N. Zakharov, Z. Liliental-Weber, B. Wagner, Z. J. Reitmeier, E. A. Preble, R. F. Davis
}

Proc. MRS 798, 747, 2004

Plan-view and cross-section samples of (1120) (a-plane) GaN grown on 4H-SiC substrates with AIN buffer layers were studied by transmission electron microscopy. Samples reveal the presence of a high density of stacking faults formed on the basal plane of hexagonal GaN. These stacking faults, terminated in the growth plane by threading dislocations, nucleate at the $\mathrm{AIN} / 4 \mathrm{H}-\mathrm{SiC}$ interface and propagate to the $\mathrm{GaN}$ layer surface. High resolution electron microscopy shows that the majority of stacking faults are low-energy planar defects of the type I/sub $1 /$ and I/sub 2/. High energy stacking faults are not observed.

\section{Elevated Temperature Mechanical Properties of Devitrified Metallic Glass}

\author{
N. A. Mara, A. V. Sergueeva, A. K. Mukherjee
}

Proc. MRS 821, 5.8, 2004

Elevated temperature tensile tests of different microstructures arising from different heat treatments of the Fe-based metallic glass Vitroperm (Fe73.5Cu1Nb3Si15.5B7) are presented. An anneal at $600 \mathrm{C}$ for $1 \mathrm{~h}$ yields a single phase a-Fe microstructure with equiaxed, randomly oriented $15 \mathrm{~nm}$ grains, which is an ideal candidate for study of material properties at diminishing length scale. This microstructure has good stability during tensile testing at $600 \mathrm{C}$, showing a strain rate exponent correlating to grain boundary sliding $(\mathrm{m}=0.5)$, but little ductility, and strengths to $1250 \mathrm{MPa}$. The brittle behavior could be attributed to the lack of dislocation activity at such length scales. At temperatures up to $725 \mathrm{C}$, grain growth occurs, leading to elongations as large as $65 \%$ at flow stresses of $250 \mathrm{MPa}$. Precipitation of a second $\mathrm{Nb}$-rich phase accompanies the grain growth. This investigation is supported by NSF, Division of Materials Research, grant NSF-DMR-0240144. 


\title{
Structural Study of V-like Columnar Inversion Domains in AIN Grown on Sapphire
}

\author{
J. Jasinski, T. Tomaszewicz, Z. Liliental-Weber, Q. S. Paduano, D. W. Weyburne
}

Proc. MRS 798, Y.5.29.1, 2004

V-like columnar inversion domains with a divergence angle of about $4.5 \mathrm{o} \pm 1 \mathrm{o}$ grown in AlN films with N-polarity were studied using transmission electron microscopy (TEM) and atomic force microscopy. Such domains emerge at the surface, forming small islands in form of hexagonal, truncated pyramids. A model of such pyramid was proposed. TEM studies indicate a displacement of $\mathrm{c} / 2$ along the [0001] direction at the inversion domain boundary. A boundary itself is composed of long segments located on the $\{1100\}$ planes, which are alternated by short segments on some inclined planes.

\section{Manifestation of Structural Defects in Photoluminescence from GaN}

\author{
M. A. Reshchikov, F. Yun, J. Jasinski, Z. Liliental-Weber, H. Morkoc
}

Proc. MRS 798, Y.5.66.1, 2004

Sharp peaks of unidentified nature are detected in the low-temperature photoluminescence (PL) spectrum of undoped $\mathrm{GaN}$ in the photon energy range between 3.0 and $3.46 \mathrm{eV}$. These PL lines are commonly attributed to excitons bound to yet unidentified structural defects. We analyzed Xray diffraction data in a large set of $\mathrm{GaN}$ samples grown by molecular beam epitaxy in order to find any correlation between these unusual PL peaks and the GaN crystal structure. Moreover, in selected samples exhibiting such peaks, cross-sectional transmission electron microscopy was taken in an effort to detect the presence and density of various structural defects. The preliminarily results indicate that most of unusual PL lines in GaN (Y lines) are not directly related to the observed structural defects, such as edge, screw, mixed dislocations, or stacking faults. However, there exists the possibility that point defects trapped at dislocations or other structural defects are responsible for these PL lines. 


\title{
TEM Studies of Ge Nanocrystals
}

Q. Xu, I.D. Sharp, C.Y. Liao, D.O. Yi, A.M. Minor, J.W. Ager III, J.W. Beeman, K.M. Yu, D.N. Zakharov, Z. Liliental-Weber, D. C. Chrzan, E.E. Haller

Proc. MRS 818, 227, 2004

Ge nanocrystals were formed by ion beam synthesis in $\mathrm{SiO}_{2}$. Transmission Electron Microscopy was used to characterize the structure and properties of these Ge nanocrystals before and after liberation from the matrix. The liberation from the $\mathrm{SiO}_{2}$ matrix was achieved through selective etching in a HF bath. High-resolution micrographs and selective area diffractionconfirm that the crystallinity is retained in this process. Transfer of released nanocrystals is achieved through ultrasonic dispersion in methanol and deposition onto lacey carbon films via evaporation of methanol. In an effort to determine the melting point of Ge nanocrystals and observe the growth and evolution of nanocrystals embedded in the amorphous $\mathrm{SiO}_{2}$ during heat treatment, as-grown nanocrystals were heated in-situ up to $1192^{\circ} \mathrm{C} \pm 60^{\circ} \mathrm{C}$ in a JEOL $200 \mathrm{CX}$ analytical electron microscope. Electron diffraction patterns are recorded using a Charge-Coupled Device. A large melting hysteresis was observed around the melting temperature of bulk Ge.

\section{Nanoscale Materials and Modeling-Relations Among Processing, Microstructure and Mechanical Properties}

\author{
D. H. Alsem, E. A. Stach, C. L. Muhlstein, M. T. Dugger, R. O. Ritchie
}

Proc. MRS 821, 2.5, 2004

Wear and fatigue are important factors in determining the reliability of microelectromechanical systems (MEMS). While the reliability of MEMS has received extensive attention, the physical mechanisms responsible for these failure modes have yet to be conclusively determined. In our work, we use a combination of on-chip testing methodologies and electron microscopy observations to investigate these mechanisms. Our previous studies have shown that fatigue in polysilicon structural thin films is a result of a 'reaction-layer' process, whereby high stresses induce a room-temperature mechanical thickening of the native oxide at the root of a notched cantilever beam, which subsequently undergoes moisture-assisted cracking. New in vacuo data show that these silicon films do not display fatigue behavior when the post release oxide is prevented from growing, because of the absence of oxygen. In particular, we have developed in vacuo and in situ experiments in the scanning electron microscope, with the objective of eventually determining the mechanisms causing both wear development and debris generation. 


\title{
Synthesis of Magnetic Self-Assembled Nickel-Rich Oxide Nanowires Using a Novel Electrochemical Process
}

\author{
Y. V. Bhargava, S. A. Thorne, T. S. Mintz, T. C. Hyams, V. Radmilovic, Y. Suzuki, T. M. Devine
}

Proc. MRS 877E, S7.4.1, 2005

We present a novel self-assembly nanowire synthesis technique capable of producing nickelrich oxide nanowires of lengths up to $20 \mu \mathrm{m}$ and diameters as small as $5 \mathrm{~nm}$. The method was discovered while examining the oxidation of Alloy 600 (nickel- $15.5 \mathrm{a} / \mathrm{o} \mathrm{Cr}, 8 \mathrm{a} / \mathrm{o} \mathrm{Fe}$ ) in a pressurized water reactor environment. The nanowires have been grown on substrates of Alloy 600 and other nickel-chromium substrates exposed to oxidizing conditions in 1500psi pressurized water with $2 \mathrm{ppm}$ lithium and $1200 \mathrm{ppm}$ boron at temperatures ranging from $238^{\circ} \mathrm{C}$ to $288^{\circ} \mathrm{C}$. Preliminary TEM analysis has revealed the nanowires to be single crystalline with an aspect ratio up to 1000:1. The nickel-rich oxide nanowires are particularly exciting because of their functional properties. Most importantly, analysis with a superconducting quantum interference device (SQUID) magnetometer indicates that the nickel-rich oxide nanowires are ferromagnetic with a coercivity of approximately $85 \mathrm{Oe}$ and a remnant field of $0.032 \mathrm{emu} / \mathrm{g}$ at $300 \mathrm{~K}$.

\section{Impacts of Back Surface Conditions on the Behavior of Oxygen in Heavily Arsenic Doped Czochralski Silicon Wafers}

\author{
Q. Wang, M. Daggubati, H. Paravi, R. Yu, X. F. Zhang
}

Proc. MRS 864E, 9.18.1, 2005

The precipitation of interstitial oxygen (Oi) in heavily arsenic doped Czochralski (CZ) silicon wafers (As-wafer) has been studied for both polysilicon and damaged back surfaces. After annealed at $1200^{\circ} \mathrm{C}$ for 45 minutes and $950^{\circ} \mathrm{C}$ for $15 \mathrm{hrs}$ sequentially, the As-wafers with polysilicon show no Oi precipitation in the bulk while polyhedral Oi precipitates are observed at the interface between polysilicon and the silicon substrate. They exhibit a habit plane of $\{100\}$. The lack of the Oi precipitation in the bulk may reduce the total gettering efficiency of the polysilicon layer on the As-wafer. The same annealing led to rod-like SiOx precipitates in the wafers with damaged back surface. These precipitates extended about 1 um into the bulk and had a habit plane of $\{111\}$. This morphology has high interfacial energy and is only possible when strain relief is dominant. The Oi outdiffusion has been observed to be same for both backside surface conditions and is only determined by annealing process 


\title{
Passive Layer Formation at Ferroelectric $\mathrm{PbTiO}_{3} / \mathrm{Pt}$ Interfaces Studied by EELS
}

\author{
S. J. Welz1, L. F. Fu1, R. Ernil, M. Kurasawa, P. C. McIntyre, N. D. Browning
}

Proc. MRS 875, 14.3.1, 2005

Polarization fatigue with repeated electric cycles in ferroelectric thin films is a major degradation problem in ferroelectric nonvolatile memories. However, the origin of this phenomenon is still not properly understood. The fatigue mechanism of a ferroelectric perovskite in a multilayer ferroelectric $\mathrm{PbTiO}_{3}$ thin film material has been investigated here using scanning transmission electron microscopy (STEM). Z-contrast images of the interfaces show that the ferroelectric $\mathrm{PbTiO}_{3}$ layer has partly decomposed into a single crystal $\mathrm{PbTiO}_{3}$ layer and an amorphous layer. Electron energy-loss spectroscopy (EELS) analysis reveals that the amorphous layer is a Ti-rich phase between $\mathrm{TiO}_{2}$ and $\mathrm{PbTiO}_{3}$. The precipitates are determined to be a Pt-Pb rich crystalline phase. It is suggested that the formation of the structure-distorted intermediate layer and precipitates may be associated with the ferroelectric degradation process by acting as a passive layer in a ferroelectric capacitor. In addition, the formation of the $\mathrm{Pt}-\mathrm{Pb}$ rich precipitates may cause an interruption of the consistent Pt electrode, which may result in failure of the device.

\section{Hafnium-Related Photoluminescence in Single Crystal Silicon}

\author{
R. Sachdeva, A. A. Istratov, Wei Shan, P. N. K. Deenapanray, E. R. Weber
}

Proc. MRS 866, 1, 2005

A new photoluminescence (PL) band in the energy range of $700 \mathrm{meV}$ to $950 \mathrm{meV}$ associated with hafnium implanted in silicon is reported. A shift in the position of photoluminescence peaks observed on the samples implanted with two different isotopes of Hf confirms the Hf-related origin of the observed photoluminescence band. Activation of the Hf-optical centers re-quires a $1000^{\circ} \mathrm{C}$ anneal step. The intensity of the PL lines depends on the cooling conditions. The spectrum consists of five peaks in the rapidly quenched sample as opposed to twenty one in the slowly cooled sample. Temperature dependent PL measurements and hydrostatic pressure measurements were performed to identify their nature. 


\title{
Synthesis and Biomedical Applications of AuFe-C Core-shell Nanoparticles
}

\author{
H. Li, H. Dai, L. Xing, R. Sinclair
}

Proc. MRS, 2006, In Press

Gold iron alloy nanoparticles about $10 \mathrm{~nm}$ in diameter enclosed by graphitic carbon are successfully synthesized by solution chemistry, Chemical Vapor Deposition (CVD) and subsequent purification. High Resolution Transmission Electron Microscopy (HRTEM) and Energy Dispersive X-ray Spectroscopy (EDS) analysis indicate that in each particle, the core is made of AuFe alloy (Au to Fe ratio is about 2:1) and the shell typically is composed of a few atomic layers of graphitic carbon. To the best of our knowledge, this is the first reported macroscopic- scale synthesis of gold-containing metal-graphitic carbon core-shell structure at nanometer scale. The nanoparticles are found to be superparamagnetic; the blocking temperature is about $80 \mathrm{~K}$. Water suspension of such particles is found to be a good absorption medium for Xrays employed in Computed Tomography (CT) imaging. It is believed that functionalized AuFe$\mathrm{C}$ nanoparticles are very promising multimodality contrast agent (Magnetic Resonance Imaging and CT) and therapeutic agent (radiation therapy and magnetic hyperthermia therapy).

\section{Optical Routing and Sensing with Nanowire Assemblies}

D. J. Sirbuly, M. Law, P. Pauzauskie, H. Q. Yan, A. V. Maslov, K. Knutsen, C. Z. Ning, R. J. Saykally, P. D. Yang

Proc. National Academy of Sciences 102, 7800, 2005

The manipulation of photons in structures smaller than the wavelength of light is central to the development of nanoscale integrated photonic systems for computing, communications, and sensing. We assemble small groups of freestanding, chemically synthesized nanoribbons and nanowires into model structures that illustrate how light is exchanged between subwavelength cavities made of three different semiconductors. The coupling strength of the optical linkages formed when nanowires are brought into contact depends both on their volume of interaction and angle of intersection. The fraction of the guided wave traveling outside the wire/ribbon cavities is used to link nanowires through space and to separate colors within multiribbon networks. We find that nanoribbons function efficiently as waveguides in liquid media and provide a unique means for probing molecules in solution or in proximity to the waveguide surface. Our results lay the spadework for photonic devices based on assemblies of active and passive nanowire elements and presage the use of nanowire waveguides in microfluidics and biology. 


\title{
TGF- $\alpha$ Signaling Regulates Mechanical Properties and Composition of Bone Matrix
}

\author{
G. Balooch, M. Balooch, R. K. Nalla, S. Schilling, E. Filvaroff, G. W. Marshall, S. J. Marshall, \\ R. O. Ritchie, R. Derynck, T. Alliston
}

Proc. National Academy of Sciences 102, 18813, 2005

The characteristic toughness and strength of bone result from the nature of bone matrix, the mineralized extracellular matrix produced by osteoblasts. The mechanical properties and composition of bone matrix, along with bone mass and architecture, are critical determinants of a bone's ability to resist fracture. Several regulators of bone mass and architecture have been identified, but factors that regulate the mechanical properties and composition of bone matrix are largely unknown. We used a combination of high-resolution approaches, including atomic-force microscopy, x-ray tomography, and Raman microspectroscopy, to assess the properties of bone matrix independently of bone mass and architecture. Smad3 $+/-$ mice had increased bone mass and matrix properties, suggesting that the osteopenic Smad3-/- phenotype may be, in part, secondary to systemic effects of Smad3 deletion. Thus, a reduction in TGF- signaling, through its effector Smad3, enhanced the mechanical properties and mineral concentration of the bone matrix, as well as the bone mass, enabling the bone to better resist fracture.

\section{In Situ Characterization of Ge Nanocrystals Near the Growth Temperature}

I. D. Sharp, Q. Xu, D. O. Yi, C. Y. Liao, J. W. Ager III, J.W. Beeman, K. M. Yu, D. N. Zakharov, Z. Liliental-Weber, D. C. Chrzan, E.E. Haller

Proc. 27th International Conference on the Physics of Semiconductors, 2004

We present in situ electron diffraction data indicating that Ge nanocrystals embedded in a silica matrix can be solid at temperatures exceeding the bulk Ge melting point. Supercooling is observed when returning from temperatures above the melting point of the Ge nanocrystals. Since melting point hysteresis is observed, it is not clear if nanoclusters are solid or liquid during the initial growth process. Raman spectra of as-grown nanocrystals give a measure of compressive stress and in-situ Raman spectroscopy further confirms the presence of crystalline $\mathrm{Ge}$ above $800^{\circ} \mathrm{C}$. 


\title{
AIP Conference Proceedings Review of Progress in Quantitative Nondestructive Evaluation: Volume 21
}

\author{
T.K. Lee, J. W. Morris, Jr., S. Lee, J. Clarke
}

Review of Progress in Quantitative Nondestructive Evaluation, 2006

The remanence fields of fatigued ferritic steel specimens were measured using a scanning microscope based on a high transition temperature Superconducting Quantum Interference Device (SQUID). The results show an overall increase of remanence until dislocation density saturates and an additional local remanence increase after saturation during cyclic loading. Because of the combined magnetic and spatial resolution of the SQUID microscope, these local changes of dislocation structures can be detected before a crack actually initiates, and identify the sites where crack nucleation will occur.

\section{Nanoribbon Waveguides for Subwavelength Photonics Integration}

\author{
M. Law, D. J. Sirbuly, J. C. Johnson, J. Goldberge, R. J. Saykally, P. D. Yang
}

Science 305, 1269, 2004

\begin{abstract}
Although the electrical integration of chemically synthesized nanowires has been achieved with lithography, optical integration, which promises high speeds and greater device versatility, remains unexplored. We describe the properties and functions of individual crystalline oxide nanoribbons that act as subwavelength optical waveguides and assess their applicability as nanoscale photonic elements. The length, flexibility, and strength of these structures enable their manipulation on surfaces, including the optical linking of nanoribbon waveguides and other nanowire elements to form networks and device components. We demonstrate the assembly of ribbon waveguides with nanowire light sources and detectors as a first step toward building nanowire photonic circuitry.
\end{abstract}




\title{
Vortex Core-Driven Magnetization Dynamics
}

\author{
S. B. Choe, Y. Acremann, A. Scholl, A. Bauer, A. Doran, J. Stohr, H. A. Padmore
}

Science 304, 5669, 2004

Time-resolved x-ray imaging shows that the magnetization dynamics of a micron-sized pattern containing a ferromagnetic vortex is determined by its handedness, or chirality. The out-of-plane magnetization in the nanometer-scale vortex core induces a three-dimensional handedness in the planar magnetic structure, leading to a precessional motion of the core parallel to a subnanosecond field pulse. The core velocity was an order of magnitude higher than expected from the static susceptibility. These results demonstrate that handedness, already well known to be important in biological systems, plays an important role in the dynamics of microscopic magnets.

\section{Interface Structure and Atomic Bonding Characteristics in Silicon Nitride Ceramics}

\author{
A. Ziegler, J. C. Idrobo, M. K. Cinibulk, C. Kisielowski, N. D. Browning, R. O. Ritchie
}

Science $306,1768,2004$

Direct atomic resolution images have been obtained that illustrate how a range of rare-earth atoms bond to the interface between the intergranular phase and the matrix grains in an advanced silicon nitride ceramic. It has been found that each rare-earth atom bonds to the interface at a different location, depending on atom size, electronic configuration, and the presence of oxygen at the interface. This is the key factor to understanding the origin of the mechanical properties in these ceramics and will enable precise tailoring in the future to critically improve the materials' performance in wide-ranging applications. 


\title{
Ultrahigh Strength in Nanocrystalline Materials Under Shock Loading
}

E. M. Bringa, A. Caro, Y. M. Wang, M. Victoria, J. M. McNaney, B. A. Remington, R. F. Smith, B. R. Torralva, H. Van Swygenhoven

Science 309, 1838, 2005

Molecular dynamics simulations of nanocrystalline copper under shock loading show an unexpected ultrahigh strength behind the shock front, with values Lip to twice those at low pressure. Partial and perfect dislocations, twinning, and debris from dislocation interactions are found behind the shock front. Results are interpreted in terms of the pressure dependence of both deformation mechanisms active at these grain sizes, namely dislocation-based plasticity and grain boundary sliding. These simulations, together with new shock experiments on nanocrystalline nickel, raise the possibility of achieving ultrahard materials during and after shock loading.

\section{Strain-compensated Nano-clusters in Al-Si-Ge Alloys}

\author{
V. Radmilovic, M. Miller, D. Mitlin, U. Dahmen
}

Scripta Mat. 54, 1973, 2006

Atom probe tomography and high resolution transmission electron microscopy have been employed to reveal clustering of Si and Ge atoms in ternary Al-Si-Ge. No such clusters were observed in binary Al-Si. The clusters were on the order of five nanometers in diameter and contained $\mathrm{Si}, \mathrm{Ge}$ and $\mathrm{Al}$. This confirms a previous hypothesis that postulates the existence of such clusters due to atomic mismatch strain compensation between the $\mathrm{Si}$ and Ge atoms in an $\mathrm{Al}$ solid solution. 


\title{
Gettering in Silicon-on-Insulator Wafers: Experimental Studies and Modeling
}

\author{
A. Istratov, H.Vainola, W. Huber, E.R.Weber
}

Semiconductor Science and Technology 20, 568, 2005

Buried oxide, which separates the device area from the substrate in silicon-on-insulator (SOI) wafers, forms a diffusion barrier for transition metals in silicon. The impact of this barrier on the efficiency of traditional gettering techniques for iron and copper is evaluated using computer modeling. Several parameters essential for the modeling, such as the diffusivity of iron in $\mathrm{SiO}_{2}$ and the segregation coefficient of iron and copper in $\mathrm{SiO}_{2}$, are verified experimentally. It is found that all available data for the diffusivity of iron $\mathrm{in}_{\mathrm{SiO}_{2}}$ (including data points from the literature and our own value of $1.410^{-13} \mathrm{~cm} \mathrm{~s}^{-2}$ at $\left.1100^{\circ} \mathrm{C}\right)$ could be fitted by the equation $\mathrm{D}(\mathrm{Fe}$ in $\left.\mathrm{SiO}_{2}\right)=2.210^{-2} \exp \left(-3.05 \mathrm{eV} / \mathrm{k}_{\mathrm{B}} \mathrm{T}\right)\left(\mathrm{cm}^{2} \mathrm{~s}^{-1}\right)$. The solubility of iron in silicon dioxide was found to be 4.5 times to 5.5 times less than that in silicon at temperatures from $1020^{\circ} \mathrm{C}$ to $1100^{\circ} \mathrm{C}$, which indicates that iron does not segregate in $\mathrm{SiO}_{2}$. Copper has sufficiently high diffusivity in $\mathrm{SiO}_{2}$ to diffuse through the buried oxide within a short time at $1000^{\circ} \mathrm{C}$. Possible gettering strategies for the SOI wafers are discussed.

\section{Surface Oxidation State of Combustion-synthesized $\gamma-\mathrm{Fe}_{2} \mathrm{O}_{3}$ Nanoparticles Determined by Electron Energy Loss Spectroscopy in the Transmission Electron Microscope}

\author{
J. Jasinski, K. E. Pinkerton, I. M. Kennedy, V. J. Leppert
}

Sens. and Actuators B 109, 19, 2005

Electron energy loss spectroscopy (EELS) in the transmission electron microscope (TEM) was used to compare the iron oxidation state at the surface and interior of gamma- $\mathrm{Fe}_{2} \mathrm{O}_{3}$ nanoparticles produced by the combustion process under fuel conditions leading to low and high soot concentrations. The studies showed that the surface oxidation state of iron was lowered with the addition of acetylene, although the core composition remained the same. This was indicated by changes in both the iron $\mathrm{L}_{23}$ - and the oxygen K-edges at the surface of the particles. These highly spatially resolved measurements showed a chemical shift of both the $\mathrm{L}_{3}$ and $\mathrm{L}_{2}$ iron lines, accompanied by significant reduction of the $\mathrm{L}_{3}: \mathrm{L}_{2}$-intensity ratio, indicating $\mathrm{Fe}^{2+}$ at the particle surface. Reduction in the pre-edge peak of the oxygen K-edge at the particle surface also indicated iron reduction at the surface. These results suggest that the surface oxidation state, and therefore gas-sensing properties, of combustion-synthesized iron oxide nanoparticles, is highly dependent on flame conditions. 


\title{
Proceedings of the 16th European Conference of Fracture
}

\author{
D. H. Alsem, R. Timmerman, E. A. Stach, M. T. Dugger, R. O. Ritchie
}

16th European Conference of Fracture: Failure Analysis of Nano and Engineering Materials and Structures, 2005

Wear and fatigue are large issues in the reliability of microelectromechanical systems (MEMS). Sliding wear debris investigations have been conducted on polysilicon side-wall friction devices run in ambient air. Electron microscopy techniques were used to image and analyze wear debris and surfaces. Comparing abrasive wear tracks, wear debris, grain size and surface roughness, it is apparent that after an initial adhesive wear mechanism, where $\sim 100 \mathrm{~nm}$ particles are removed from the surface by fracture through the grains $(\sim 500 \mathrm{~nm})$, an abrasive wear mechanism regime occurs and creates wear plough tracks with widths similar to the size of debris particle agglomerates. Of note is the fact that the debris particles are amorphous and have high oxygen content, but do not completely consist of $\mathrm{SiO}_{2}$. This observation, coupled with the fact that postrelease oxide layers in these films are only $\sim 3 \mathrm{~nm}$ thick, implies that the debris particles are formed by silicon from the film that amorphizes and subsequently partly oxidizes.

\section{Characterization of Interfaces on the Atomic Scale with the Conjunction of Stem Z-Contrast Imaging and EELS}

\author{
L. F. Fu, N. D. Browning, D. C. Kundaliya, S. X. Zhang, W. Ramadan, T. Venkatesan, S. B. \\ Ogale
}

6th Pacific Rim Conference on Ceramic \& Glass Technology, 2005

\begin{abstract}
Interfaces between two dissimilar materials have long been known to dominate the performance of many heterointerface systems. Microstructural characterizations at the highest spatial resolution have always been a subject of great scientific interest to understand the physical properties of these systems. Commercially available high spatial resolution scanning transmission electron microscopes (STEM) have provided the possibility of widespread applications of electron microscopic characterization techniques for interface studies. For instance, the atomic and electronic structure of interfaces can be studied directly without computer simulation or reconstruction using Z-contrast imaging and EELS. In this paper, we discuss the practical aspects of the experimental STEM techniques and demonstrate the application of these techniques to the study of heterophase interfaces and defects in $\mathrm{YBCOSrTiO}_{3}, \mathrm{Co}: \mathrm{TiO}_{2}-\mathrm{LaAlO}_{3}$, and $\mathrm{Co}: \mathrm{HfO}_{2}$-YSZ ceramic-oxides interface systems.
\end{abstract}




\title{
Thermally Driven Interfacial Dynamics of Metal/Oxide Bilayer Nanoribbons
}

\author{
M. Law, X. F. Zhang, R. Yu, T. K. Kendall, P. D. Yang
}

Small 1,1, 2005

Solid-solid interfacial processes greatly affect the performance of electronic and composite materials, but probing the dynamics of buried interfaces is challenging and often involves lengthy or invasive sample preparation. We show that bilayer nanoribbons / made here of tin dioxide and copper / are convenient structures for observing as-made interfaces as they respond to changing temperature in a transmission electron microscope (TEM). At low temperatures $\left(\angle 200^{\circ} \mathrm{C}\right)$, differential thermal expansion causes the bilayers to bend when heated or cooled, with the motion determined by the extent of $\mathrm{Cu}-\mathrm{SnO}_{2}$ epitaxy. At higher temperatures, we are able to watch, in real time and with nanometer resolution, a progression of grain growth, interdiffusion, island formation, solid-state chemical reactions and melting. This novel TEM geometry is readily applicable to other nanoribbon/coating combinations and is well suited to observing interfacial phenomena driven thermally or by the application of mechanical, electrical or magnetic forces.

\section{Local Indium Segregation and Band Gap Variations in High Efficiency Green Light Emitting InGaN/GaN Diodes}

\author{
J.R. Jinschek, R. Erni, N.F. Gardner, A.Y. Kim, C. Kisielowski
}

Solid State Comm. 137, 4, 230, 2006

High efficient green light emitting diodes (LED) on the basis of $\mathrm{GaN} / \mathrm{InGaN}$ exhibit indium-rich nanoclusters inside the quantum wells (QW) due to InN-GaN phase decomposition. By direct measurements of the variations in the electronic structure, we show for the first time a correlation between indium-rich nanoclusters and local energy band gap minima. Our investigations reveal the presence of 1-3 nm wide indium rich clusters in these devices with indium concentrations $\mathrm{x}$ as large as $\mathrm{x}$ similar to $0.30-0.40$ that narrow the band gap locally to energies as small as 2.65 $\mathrm{CV}$. These clusters are able to act as local traps for migrating photon-emitting carriers and seem to boost the overall device performance. 


\title{
Experimental Study on Re-Dissolution of Gettered Iron Impurities in Czochralski-Grown Silicon
}

\author{
P. Zhang, H. Vainola, A. Istratov, E. R. Weber
}

Solid State Phenomena 95, 577, 2004

In order to control the degradation of silicon devices by metal impurities, especially transition metals, different gettering techniques were developed in the past decades. The transition metal gettering by oxide precipitates formed in the bulk wafer turns out to be efficient for fast diffusers such as iron. However, the gettered iron impurities can re-dissolve after gettering into the silicon matrix during the thermal cycles and very little is known about this dissolution process. In this paper, we report a systematic study of the re-dissolution behavior of gettered iron impurities in ptype Czochralski-grown silicon with doping level about $2.5 \times 10^{14} \mathrm{~cm}^{-3}$ and oxide precipitates density of $5 \times 10^{9} \mathrm{~cm}^{-3}$. The concentration of interstitial iron and iron-boron pairs is measured by deep level transient spectroscopy (DLTS). It is found that the dependence of the re-dissolved iron concentration on annealing time can be fitted empirically by the function $\mathrm{C}(\mathrm{t})=\mathrm{C}_{\mathrm{o}}(1-\mathrm{e}(-$ $1 / \tau)$ ), and $\tau^{-1}$ has an Arrhenius dependence on temperature: $\tau^{-1}=4.01 \times 10^{4} \times \exp (-$ $1.47 \mathrm{eV} / \mathrm{k}(\mathrm{B}) \mathrm{T}) / \mathrm{s}$

\section{Physics and Technology of Thin Films}

\section{Farle}

Some Apects in Thin Film Magnetism, 441, 2004

Research on magnetic mono- und multilayers has provided a better understanding of the microscopic mechanisms which determine macroscopically observable quantities like the magnetization, different types of magnetic order (ferro- , ferri- and antiferromagnetism), magnetic anisotropy, ordering temperatures (Curie, Néel temperature) and exchange coupling. Aside from these basic research orientated investigations the technological exploitation of thin film magnetism has lead to huge increases in the hard disk's magnetic data storage capacities and new types of magneto-resistive angle and position sensitive sensors in the automotive industry, for example. The technological important aspects of exchange biased, spin- valve, or exchangecoupled multilayer structures will not be discussed here, however. In this contribution I will discuss some aspects of thin film magnetism dealing mainly with the variation of magnetic anisotropy energy (MAE) as a function of temperature and film thickness and its importance for the understanding of so-called spin reorientation phase transitions. 


\title{
Surface-Energy-Anisotropy-Induced Orientation Effects on Rayleigh Instabilities in Sapphire
}

\author{
M. Santala, A. Glaeser
}

Surface Science 600, 782, 2006

Arrays of controlled-geometry, semi-infinite pore channels of systematically varied crystallographic orientation were introduced into undoped m-plane (10-10) sapphire substrates using microfabrication techniques and ion-beam etching and subsequently internalized by solidstate diffusion bonding. A series of anneals at $1700^{\circ} \mathrm{C}$ caused the breakup of these channels into discrete pores via Rayleigh instabilities. In all cases, channels broke up with a characteristic wavelength larger than that expected for a material with isotropic surface energy, reflecting stabilization effects due to surface-energy anisotropy. The breakup wavelength and the time required for complete breakup varied significantly with channel orientation. For most orientations, the instability wavelength for channels of radius $R$ was in the range of $13.2 \mathrm{R}-25 \mathrm{R}$, and complete breakup occurred within 2-10 h. To first order, the anneal times for complete breakup scale with the square of the breakup wavelength.

\section{Substrate Effects on the Creep Properties of Pure Sn Solder Joints}

\author{
K. O. Lee, J. W. Morris, Jr., F. Hua
}

10th International Symposium on Advanced Packaging Materials, (Chapter: Solder Composition), 2005

This study investigated the influence of substrate metallization on the microstructure and creep behavior of the pure tin solder joints. Both symmetric $(\mathrm{Cu}: \mathrm{Cu})$ and the asymmetric $(\mathrm{Cu}: \mathrm{Au} / \mathrm{Ni})$ metallizations were tested. The solder joints with asymmetric substrates had lower creep rates than those with symmetric $(\mathrm{Cu}: \mathrm{Cu})$ substrates. $(\mathrm{Cu}-\mathrm{Ni}) / \sup 6 / \mathrm{Sn} / \mathrm{sup} 5 /$ intermetallics formed on all substrates. However, a dramatic and anomalous intermetallic growth was observed on the Niside of the asymmetric joints; after typical reflow the intermetallic reached almost one third of the solder joint thickness. It appears that the abnormally thick intermetallic inhibits creep in the asymmetric joint and is the primary cause of the lower creep rates. 


\title{
Materials Fundamentals of Gate Dielectrics
}

\author{
A. Navrotsky, S. V. Ushakov
}

Thermodynamics of oxide systems relevant to alternative gate dielectrics, 57-108, 2005

The search for gate dielectric materials superior to amorphous silica requires the input of thermodynamic data to assess materials compatibility with silicon. Because superior dielectric properties are generally associated with materials containing heavy ions of large size and high charge, emphasis has been on trivalent and tetravalent oxides containing $\mathrm{Ti}, \mathrm{Zr}$, Hf, and the rare earths. Furthermore, the materials must be insulating and the oxide must be less reducible than $\mathrm{SiO}_{2}$ so that it does not oxidize silicon. Within these constraints, an amorphous film is considered superior to a polycrystalline one with grain boundaries, so it is important to understand the persistence, controlled by both thermodynamic and kinetic factors, of amorphous and glassy materials. The purpose of this paper is to summarize relevant thermodynamic data, to provide a thermodynamic and structural framework for considering new compositions and their likely properties, and to present some new calorimetric data on bulk and thin film systems based on $\mathrm{ZrO}_{2}$ and $\mathrm{HfO}_{2}$.

\section{An Electron Microscopy Study of Wear in Polysilicon Microelectromechanical Systems}

\author{
D.H. Alsem, E.A. Stach, M.T. Dugger, M. Enachescu, R.O. Ritchie
}

Thin Solid Films, 2006, in Press

While the reliability of polysilicon MEMS has received extensive attention, the mechanisms responsible for this failure mode at the microscale have yet to be conclusively determined. We have used on-chip polycrystalline silicon sidewall friction MEMS specimens to study active mechanisms during sliding wear. Worn parts were examined by analytical scanning and transmission electron microscopy, while local temperature changes where monitored using advanced infrared microscopy. Observations show that small amorphous debris particles ( $\sim 50-$ $100 \mathrm{~nm})$ are removed by fracture through the silicon grains $(\sim 500 \mathrm{~nm})$ and are oxidized during this process. Some of these debris particles/clusters create plowing tracks on the beam surface. A nano-crystalline surface layer $(\sim 20-200 \mathrm{~nm})$, with higher oxygen content, forms during wear at and below regions of the worn surface; its formation is likely aided by high local stresses. No evidence of dislocation plasticity or of extreme local temperature increases was found, ruling out the possibility of high temperature-assisted wear mechanisms. 


\title{
Nanomechanics of Biopolymers Studied by In-situ Nanoindentation
}

\author{
J. Zhou, K. Komvopoulos \\ 3rd Annual University of California Symposium on Surface Science and Its Applications, 2005
}

Advances in biopolymer materials require basic understanding of the correlation between nanomechanical properties and the microstructure evolution. Despite numerous studies dealing with the macro- and mesoscopic mechanical behavior of biopolymers, insight into the interdependence of microstructure and mechanical response at the nanoscale is limited. This research aims to develop a direct in-situ TEM-based approach that enables observation of microstructure evolution during nanoindentation. Ion beam-assisted nanomachining was used to fabricate polymer thin-film samples suitable for in-situ TEM study. The deformation and fracture behaviors of the thin polymer films under nanoindentation loading and unloading are directly observed in the TEM.

\section{Effect of Shock Compression Method on the Defect Substructure in Monocrystalline Copper}

B. Y. Cao, M. A. Meyers, D. H. Lassila, M. S. Schneider, B. K. Kad, C.X. Huang, Yong Bo Xu, D. H. Kalantar, B.A. Remington

TMS Annual Meeting, 2005

Monocrystalline copper samples with orientations of [001] and [221] were shocked at pressures ranging from 20 to $60 \mathrm{GPa}$ using two techniques: direct drive lasers and explosively driven flyer plates. The residual microstructures were dependent on orientation, pressure, and shocking method. The much shorter pulse duration in the laser driven shock yielded microstructures in recovery samples closer to those generated at the shock front. For the flyer-plate experiments, the longer pulse duration allows shock-generated defects to reorganize into lower energy configurations. Calculations show that the post-shock cooling for the laser driven shock is $10^{\text {; }}$ _10 faster than that for plate-impact shock. At the higher pressure level, extensive recrystallization was observed in the plate-impact samples, while it was absent in laser driven shock. An effect that is proposed to contribute significantly to the formation of recrystallized regions is the existence of micro-shear-bands, which increase the local temperature beyond the prediction from adiabatic compression. 


\title{
Analysis of Nano-scale Stress in Strained Silicon Materials and Microelectronics Devices by Energy-filtered CBED
}

\author{
P. Zhang, A.A. Istratov, H. He, J.W. Ager, C. Nelson, E. Stach, J. Mardinly, C. Kisielowski, E.R. \\ Weber, J.C.H. Spence
}

209th Meeting of the Electrochemical Society, 2006

The convergent beam electron diffraction (CBED) technique of transmission electron microscopy (TEM) has excellent capabilities for strain detection at high spatial resolution. Here we report strain measurements in bulk s-Si/SiGe/Si and in a strained $35 \mathrm{~nm}$ PMOS device in which SiGe acts as the source and drain. CBED measurements of the composition of the relaxed $\mathrm{SiGe}$ buffer are in quantitative agreement with Raman spectroscopy. For the PMOS device, CBED measured a uniaxial compressive stress of $1.12 \mathrm{GPa}$ in the channel. However, it was found that even in the cross-sectional TEM samples with thicknesses greater than $300 \mathrm{~nm}$, the intrinsic surface strain relaxation was often so severe that no recognizable high-order Laue zone lines in the CBED patterns could be collected. The amorphization of both free surfaces of the TEM sample to a range of about $80 \mathrm{~nm}$ is proposed to minimize the impact of surface strain relaxation for future studies.

\section{Stacking Disorder in ZnS Nanoparticles as a Function of pH}

\author{
H. Zhang, F. Huang. B. Gilbert, J. Banfield
}

229th ACS National Meeting, San Diego, INOR-937, 2005

$\mathrm{ZnS}$ nanoparticles $(4-5 \mathrm{~nm})$ synthesized by reaction between zinc acetate and thioacetamide in aq. solns. at $\mathrm{pH}$ values ranging from 4 to 11 have different structural characteristics. X-ray diffraction (XRD) patterns from the nanoparticles synthesized in weak acidic and near neutral $\mathrm{pH}$ conditions cannot be simulated by summing patterns for pure sphalerite (cubic) and wurtzite (hexagonal) nanoparticles. Mixed sphalerite and wurtzite structure in individual nanoparticles synthesized in near neutral $\mathrm{pH}$ solns. was demonstrated by transmission electron microscope observation. A statistical structure model based on random stacking of layers was developed. We varied the probability of $\mathrm{ABA}$ vs. ABC-type stacking to account for the characteristics of the XRD patterns. In contrast, the nanoparticles synthesized at basic conditions are close to pure sphalerite. The ability to create a mixed cubic-hexagonal structure in $\mathrm{ZnS}$ nanoparticles by simply adjusting the soln. $\mathrm{pH}$ opens an easy and direct way to control the nanostructures, and hence the properties, of this optical semiconductor. 


\title{
STEM Characterization and Band Gap Measurements of HgTe/CdTe Superlattices
}

\author{
L. Fu, N. D. Browning, H. Jung, C. Grein
}

2005 U.S. Workshop on the physics and chemistry of II-VI materials, 2005

Extensive studies have been performed on II-VI narrow-bandgap quantum structures to characterize their small effective masses and energy gaps [1-6]. Among these II-VI//II-VI systems, $\mathrm{HgTe} / \mathrm{CdTe}$ superlattices (SLs) are of particular interest for both the fundamental physics and their promising applications as infrared detectors $[7,8]$. The electrical and infrared detector properties of $\mathrm{HgTe} / \mathrm{CdTe} \mathrm{SLs}$ are strongly dependent on the $\mathrm{HgTe} / \mathrm{CdTe}$ heterointerfaces. Furthermore, as the band gaps of $\mathrm{HgTe} / \mathrm{CdTe}$ SLs are related to the thickness of individual layers, an exact knowledge of the thickness variation in $\mathrm{HgTe} / \mathrm{CdTe}$ SLs and its relationship with the band gaps is important. To fully develop $\mathrm{HgTe} / \mathrm{CdTe} \mathrm{SLs}$ for infrared detector devices it is therefore necessary to characterize the properties of the heterointerfaces.

\section{Coherence and Sampling Requirements for Diffractive Imaging}

\author{
J. C. H. Spence, U. Weierstall, M. Howells
}

Ultramicroscopy 101, 149, 2004

Coherent Diffractive Imaging (CDI) allows images to be reconstructed from diffraction patterns by solving the noncrystallographic phase problem for isolated nanostructures. We show that the Shannon sampling of diffraction intensities needed in CDI requires a coherence width about twice the lateral dimensions of the object, and that the linear number of detector pixels fixes the energy spread needed in the beam. The Shannon sampling, defined by the transform of the periodically repeated autocorrelation of the object, is related to Bragg scattering from an equivalent crystal, and shown to be consistent with the sampling of Young's fringes established by scattering from extreme points in the object. The results are relevant to the design of diffraction cameras for CDI and plans for femtosecond X-ray diffraction from individual proteins. 


\title{
Nonlinear Imaging Using Annular Dark Field TEM
}

\author{
S. Bals, R. Kilaas, C. Kisielowski
}

Ultramicroscopy 104, 281 (2005)

Annular dark field TEM images exhibit a dominant mass-thickness contrast that can be quantified to extract single atom scattering cross sections. On top of this incoherent background, additional lattice fringes appear with a nonlinear information limit of 1.2 angstrom at $150 \mathrm{kV}$. The formation of these fringes is described by coherent nonlinear imaging theory and good agreement is found between experimental and simulated images. Calculations furthermore predict that the use of aberration corrected microscopes will improve the image quality dramatically.

\section{First-Principles Study on Incidence Direction, Individual Site Character, and Atomic Projection Dependences of ELNES for Perovskite Compounds}

\author{
T. Mizoguchi, J. P. Buban, K. Matsunaga, T. Yamamoto, Y. Ikuhara
}

Ultramicroscopy 106, 92, 2006

The incidence direction dependence, the individual site character dependence, and the atomic projection dependence of O-K ELNES from $\mathrm{YBa}_{2} \mathrm{Cu}_{3} \mathrm{O}_{7}$ and $\mathrm{SrTiO}_{3}$ were theoretically simulated using the first principles band structure calculation. In order to calculate ELNES, a core-hole was introduced at the oxygen 1s orbital, and a sufficient large supercells composed of more than one hundred atoms were employed. We found that the intensity of the first peak of O$\mathrm{K}$ ELNES from $\mathrm{YBa}_{2} \mathrm{Cu}_{3} \mathrm{O}_{7}$ strongly depends on the atomic projection direction, and disappears when the spectrum is measured with the other projection directions. The large projection dependence was also predicted in the O-K ELNES of $\mathrm{SrTiO}_{3}$. It was found that those spectral changes according to the position of the projection are caused by the unidirectional Ti-O-Ti bond in $\mathrm{SrTiO}_{3}$. 


\title{
Manifestations of Dynamic Strain Aging in 2090 Al-Li Alloy
}

\author{
N. Ilic, M.T. Jovanovic, V. Radmilovic, D. Drobnjak
}

Zeitschrift fur Metallkunde 96, 138, 2005

The manifestations of dynamic strain ageing in a commercial $2090 \mathrm{Al}-\mathrm{Li}$ alloy were investigated in a wide range of temperatures (-40 to 100 degrees $C)$ and strain rates $(4.16 \times 10(-5)$ to $4.16 \times 10(-3) \mathrm{s}(-1))$. Serrated yielding, as a macroscopic manifestation of dynamic strain ageing, occurs under all testing conditions. Negative strain-rate sensitivity of the flow stress is also observed. Temperature rise and strain rate reduction cause the increase of the yield strength and the work-hardening rate, as well as the decrease of elongation - the behavior being typical for the range in which C-type serrations appear. These phenomena are related to the dislocation substructure, in which features characteristic for solute atoms blocking of dislocations and Orowan bypassing are observed.

\section{Controlling Copper and Iron Precipitation in Silicon Wafers}

\author{
Hele Savin
}

Ph.D. Thesis, Helsinki University, 2005

The precipitation behavior of the two most common transition metals in silicon, copper and iron, are studied in this thesis. It is shown that external charge on wafer surfaces can reduce the copper out-diffusion, which extends the applicability of the method. Moreover, oxide precipitates are found to increase the sensitivity of the method. An analytical solution to the current continuity equation for excess carriers in an epitaxial structure under time dependent optical excitation is derived. Several annealing profiles and contamination levels are used to study the iron gettering behavior at varying supersaturation levels. It was found that the gettering efficiency depends strongly on the initial iron concentration and considerably high supersaturation is needed to initiate the gettering. The thermal stability of internally gettered iron is studied experimentally by deep level transient spectroscopy. The experiments reveal that the dissolution is a reaction limited process. The simulations of iron re-dissolution show that instead of simulating only the final cooling, it is important to simulate the total thermal cycle. 


\title{
Irradiation Induced Dislocations and Vacancy Generation in Single Crystal Yttria Stabilized Zirconia
}

\author{
J. Johnsen
}

Ph.D. Thesis, University of California, Davis, 2006

A determination of the most effective method of introducing defect clusters and forming nanocrystals in single crystal Yttria Stabilized Zirconia (YSZ) to increase its oxygen ion conductivity for use in solid oxide fuel cell has been investigated using several techniques. Highenergy particle irradiation using $800 \mathrm{keV}$ electrons and $20 \mathrm{MeV}$ protons and $\mathrm{Ar}^{+}$and $\mathrm{Xe}^{++}$ion implantation promote the introduction of defects. Thermal annealing and temperature cycling were performed both ex-situ and in-situ in a TEM to study the dynamic recovery behavior of the defects introduced by irradiation and the nucleation and growth of nanocrystals. Impedance spectroscopy measurements were conducted on proton irradiated samples with various annealing conditions. From the impedance results it is concluded that the minimum annealing conditions for a noticeable improvement in ionic conductivity are $1000^{\circ} \mathrm{C}$ for 2 hours and the $1200^{\circ} \mathrm{C}$ for 1 hour. The proton irradiated YSZ ceramic samples annealed under these conditions were found to have slightly enhanced electrical properties compared to the baseline unmodified YSZ at high temperatures. 

Please send a reprint of the paper(s):

\begin{tabular}{|l|l|l|}
\hline Number & First Author & Title (first two words) \\
\hline & & \\
\hline & & \\
\hline & & \\
\hline & & \\
\hline & & \\
\hline & & \\
\hline & & \\
\hline
\end{tabular}

Name

Date

Affiliation

Address

Please send a reprint of the paper(s):

\begin{tabular}{|l|l|l|}
\hline Number & First Author & Title (first two words) \\
\hline & & \\
\hline & & \\
\hline & & \\
\hline & & \\
\hline & & \\
\hline & & \\
\hline & & \\
\hline
\end{tabular}

Name

Date

Affiliation

Address 
National Center for Electron Microscopy Berkeley Laboratory

1 Cyclotron Rd., Building 72

Berkeley CA 94720

National Center for Electron Microscopy

Berkeley Laboratory

1 Cyclotron Rd., Building 72

Berkeley CA 94720 

National Center for Electron Microscopy Ernest Orlando Lawrence Berkeley National Laboratory

University of California Berkeley, CA 94720 